\title{
Profile and Economic Impacts of Agriculture and Natural Resource Industries in the Central Region of Florida
}

\author{
M. Rahmani, A.W. Hodges, and W.D. Mulkey \\ University of Florida/Institute of Food and Agricultural Sciences \\ Food and Resource Economics Department \\ P.O. Box 110240, Gainesville, Fl 32611-0240 \\ Ph. (352)392-1881 X 315 or 312, Fax (352)392-3646 \\ Email: rahmani@ufl.edu or awhodges@ufl.edu
}

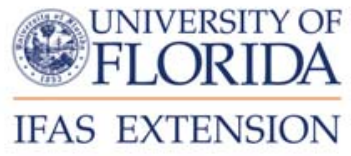

The Institute of Food and Agricultural Sciences (IFAS) is an Equal Opportunity Institution authorized to provide research, educational information and other services only to individuals and institutions that function with non-discrimination with respect to race, creed, color, religion, age, disability, sex, sexual orientation, marital status, national origin, political opinions or affiliations. U.S. Department of Agriculture, Cooperative Extension Service, University of Florida, IFAS, Florida A\&M University Cooperative Extension Program, and Boards of County Commissioners Cooperating. Larry Arrington, Dean 


\section{Executive Summary}

This report provides a profile of agricultural and natural resource industries and an overview of their economic impacts for a 20-county region in central Florida based on data from the IMPLAN Pro database for Florida counties for 2002 and data from the 2002 Census of Agriculture. Descriptive data include farm characteristics (number, distribution, farm land use, and investment in land and buildings); value of agricultural products; and measures of economic impacts such as output, valueadded, and employment. Where possible, changes during the 1997-2002 period are highlighted. Additional reports are available for other major regions of Florida.

In 2002, the central region of Florida had close to 20,000 farms and more than 5 million acres of farmland with an estimated asset value of land and buildings exceeding \$14 billion. Beef and cattle ranching and farming dominated the farm numbers in the region, while all types of pasture constituted more than 3 million acres, or 69 percent of reported land use. Major areas of agricultural activities in the central region of Florida included fruits (citrus), tree nuts, and berries; nursery, greenhouse, and sod; livestock, poultry, milk, and dairy products; and vegetables, melons, and potatoes. The market value of agricultural products sold in the central region of Florida exceeded $\$ 2.6$ billion in 2002, where the value of fruits (citrus), tree nuts, and berries constituted the highest portion, with $\$ 1.1$ billion, or more than 41 percent of the regional total. Net farm cash income from farm operations exceeded $\$ 641$ million for all farms in the region for the same period. Polk County had the highest farm numbers and estimated asset value of land and buildings, while Osceola County had the highest farm acreage and Hillsborough County had highest value of all agricultural products sold in the central region of Florida. Changes in the region during the 1997-2002 period included an 8 percent increase in the number of farms and a 12 percent decrease in cropland acreage. The market value of all agricultural products sold decreased by 3 percent between the two census periods. While the value of fruits (citrus), tree nuts, and berries increased by 5 percent, the value of all livestock, poultry and their products decreased by 9 percent during the 1997-2002 period.

The total economic impacts for all agriculture and natural resource industries in the central region of Florida exceeded $\$ 27$ billion in output in 2002. The fruit and vegetable farming and processing industry was the largest sector, contributing more than $\$ 7.6$ billion in total output impacts and accounting for 28 percent of the total in the region. More than 300,000 full- and part-time jobs were generated by agricultural and natural resource industries in the region. The agricultural input and services industry produced more than 91,000 jobs and accounted for over 30 percent of total employment impacts for all industry groups in the region. The total value-added impacts of the agricultural and natural resource industries exceeded $\$ 13.6$ billion in 2002 . Once again, the fruit and vegetable farming and processing industry with $\$ 4$ billion in value-added impacts accounted for more than 29 percent of the total value-added impacts (the highest for the region). In terms of economic impacts, Hillsborough County had the highest output impacts and value-added impacts, while Polk County had the highest employment impacts in the central region of Florida. 


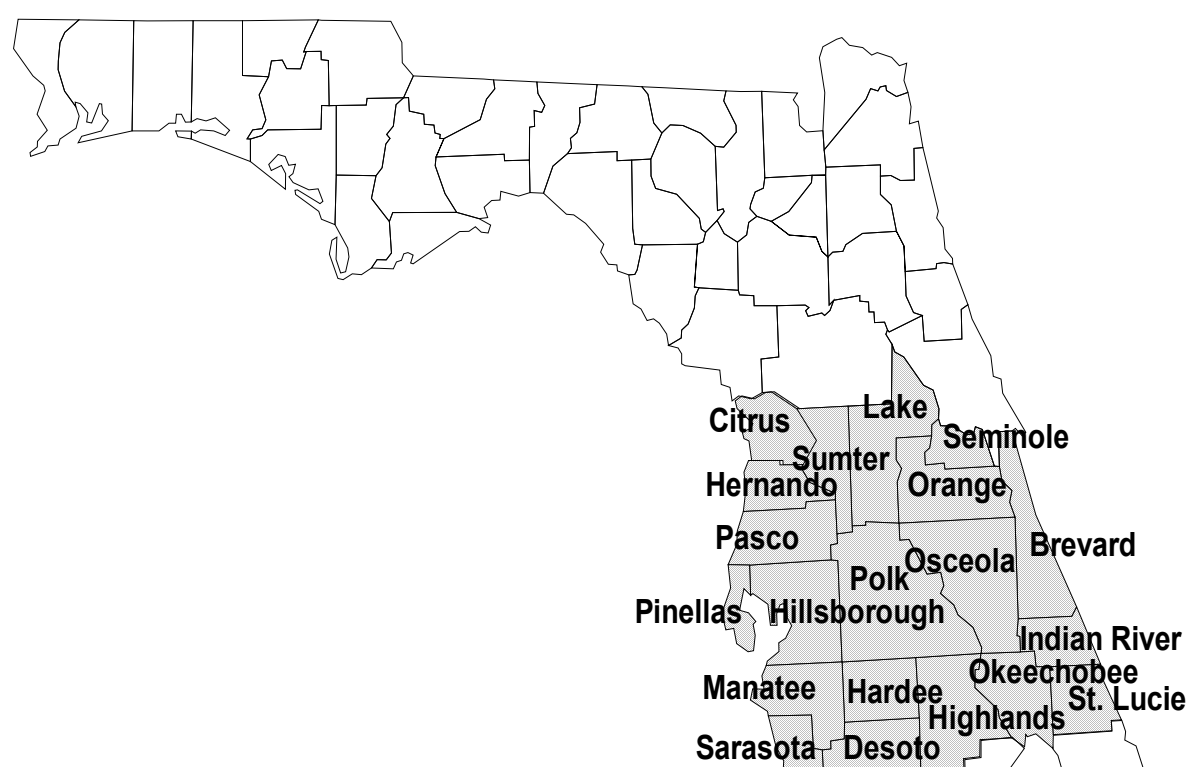

Figure 1. Study area in central region of Florida: Brevard, Citrus, DeSoto, Hardee, Hernando, Highlands, Hillsborough, Indian River, Lake, Manatee, Okeechobee, Orange, Osceola, Pasco, Pinellas, Polk, St. Lucie, Sarasota, Seminole, and Sumter Counties. 


\section{Introduction}

This report provides a profile of agriculture and natural resource industries and an overview of their economic impacts for a 20-county region in central Florida (Figure 1). Additional reports provide similar information for the northeast, northwest, south, and Suwannee River Basin areas of the state. The report is based on data from the IMPLAN Pro database for Florida counties for 2002 and data from the 2002 Census of Agriculture. ${ }^{1}$

The IMPLAN Pro Economic Impact and Social Accounting software package, licensed to the University of Florida by the Minnesota IMPLAN Group, Inc., was used to generate multipliers for output, employment, and value-added for each agricultural and natural resource sector of the regional economy to allow a more comprehensive assessment of impacts. In addition to direct sales of the industries in question, IMPLAN multipliers capture inter-industry purchases (indirect effects) and employee household spending (induced effects). Regional IMPLAN models were constructed for each county using the county-level database provided with the IMPLAN software. No attempt was made to adjust for particular local conditions, and the analysis focused only on economic impacts resulting from operating revenues and employment created by agricultural and natural resource industries.

Data from the 2002 Census of Agriculture ${ }^{2}$ were used to augment the economic impact analysis and to allow for a detailed description of agricultural and natural resource industries for counties in the region. The 2002 Census of Agriculture, conducted by the National Agricultural Statistics Service of the U.S. Department of Agriculture, provides information at the county level on farm numbers; land use; operator characteristics; the market values of land, buildings, and machinery; crop and livestock sales; farm expenses; and income from federal programs.

\section{Farm Characteristics by County}

\section{Farm Numbers (North American Industry Classification System)}

There were more than 19,600 farms in the central region of Florida in 2002, accounting for 45 percent of all farms in the state (Table 1). Beef and cattle ranching and farming constituted the largest number of farms $(7,364)$, or more than 37 percent of all farms in this region. Fruit and tree nut farming ranked second (6,465 farms, or 33 percent of the regional total), while animal aquaculture and other animal production ranked third (2,638 farms, or 13 percent of the regional total). The central region had no tobacco or cotton farms. Polk County with 3,114 farms had the highest farm numbers in the central region (16 percent of all farms in the region), followed by Hillsborough County with 2,969 farms (15 percent of all farms in the region). DeSoto, Hardee, Highlands, Lake, and Pasco Counties each had more than 1,000 farms.

\footnotetext{
${ }^{1}$ IMPLAN Professional, Social Accounting \& Impact Analysis Software, $2{ }^{\text {nd }}$ Edition, June 2000, Minnesota IMPLAN Group, Inc., 1725 Tower Drive West, Suite 140, Stillwater, Minnesota 55082, www.implan.com

22002 Census of Agriculture, Florida, State and County Data, Volume 1, Geographic Area Series, Part 9, AC-02-A-9, Issued June 2004, National Agricultural Statistics Service, U.S. Department of Agriculture.
} 
Farm numbers noted are based on the definition of a farm used by the 2002 Census of Agriculture: "any place from which $\$ 1,000$ or more of agricultural products were produced and sold, or normally would have been sold, during the census year." This definition was first used for the 1974 Census of Agriculture and has remained consistent since so that data are comparable across time periods.

\section{Farm Size Distribution and Investment in Land, Buildings, and Machinery}

The central region had more than 5,116,000 acres of land in farms, accounting for 49 percent of total farming acreage for Florida (Table 2). Within the region, Osceola County had the largest farm acreage (more than 652,000 acres, or 13 percent of all farmland in the region) and Polk County was second in the region (more than 626,000 acres, or 12 percent of the regional total). The largest average farm size was in Osceola County (1,258 acres per farm), followed by Okeechobee County (615 acres per farm). An average farm size of less than 100 acres was reported for Hillsborough, Pinellas, and Seminole Counties.

The estimated asset value of land and buildings for farms in the central region exceeded $\$ 14$ billion, accounting for 48 percent of the total value for all of Florida and for an average value of $\$ 810,000$ per farm in the region. Across the region, Polk County had the highest value for land and buildings ( $\$ 1.8$ billion), while Hillsborough County had the second highest total ( $\$ 1.3$ billion). The estimated asset value of land and buildings in Highlands and Osceola counties each exceeded $\$ 1$ billion. The average value of land and building per farm ranged from slightly more that $\$ 362,000$ in Pinellas County upwards to more than $\$ 2$ million in Osceola County

For the region as a whole, the total number of farms decreased by 8 percent, while the total farm acreage increased by 2 percent, and total asset values increased by 17 percent during the 1997-2002 period. Not all counties, however, followed the regional patterns of change. The number of farms reported in DeSoto County increased by 23 percent between the two census periods. Pinellas County had the highest farm number decrease at 33percent. Farm numbers remained almost unchanged in Highlands, Lake, Okeechobee, and Polk Counties, increased 5 percent in Citrus County, and decreased in all other counties in the region between the two census periods.

Farm numbers, however, do not always tell the entire story of changes in agriculture at the county level. The 23 percent increase in the number of farms in DeSoto County (1997-2002) is a case in point. The actual census data (not reported but summarized in Table 2) show an increase of 212 farms in DeSoto County, from 941 in 1997 to 1,153 in 2002. The 212 farm increase came primarily in the farm groups in the 1-9 acres category (61 farms) and the 10-49 acres category (123 farms). There was a smaller increase in farms in the 50-1999 acres category (28 farms). Total farm acreage in DeSoto County increased by 20 percent, and the average farm size decreased by 2 percent. These farm numbers appear to reflect the popularity of small farm development in DeSoto County.

The farm acreage reported for DeSoto County increased by 20 percent (the largest increase), and reported farm acreage in Pinellas County decreased by 44 percent (the largest decrease). Six other counties reported increases in farm acreage, while decreases were reported for 9 counties across the region. As noted earlier, the net effect at the regional level was a modest increase (2 percent) in reported farm acreage over the 1997-2002 periods. 
For the region as a whole, the estimated value of farm investments in land and buildings increased by 17 percent between 1997 and 2002. Across the region, 11 counties had percentage increases in the value of land and buildings that exceeded the average increase for the region. Manatee County had the highest increase (35 percent) in the estimated asset value of land and buildings, while the total value of land and buildings decreased in 5 counties.

\section{Farmland Use}

Agricultural lands in the central region of Florida were used mainly for cropland, nursery, greenhouse, floriculture, pasture, rangeland, and woodland. Over 1,540,000 acres were used as cropland in the region in 2002. Harvested cropland constituted 57 percent of the total cropland (Figure 2). Nursery, greenhouse, and floriculture included about 100 million square feet under glass or other protection plus close to 60,000 acres in the open. Over 3 million acres were used for all types of pasture, and there were about 780,000 acres in total woodland, with 76 percent of it used as pasture. Land enrolled in conservation or Wetlands Reserve Programs and federal or other crop insurance programs represented 307,000 acres out of a total of more than 1.5 million acres (Tables $3 \mathrm{~A} \& 3 \mathrm{~B})$.

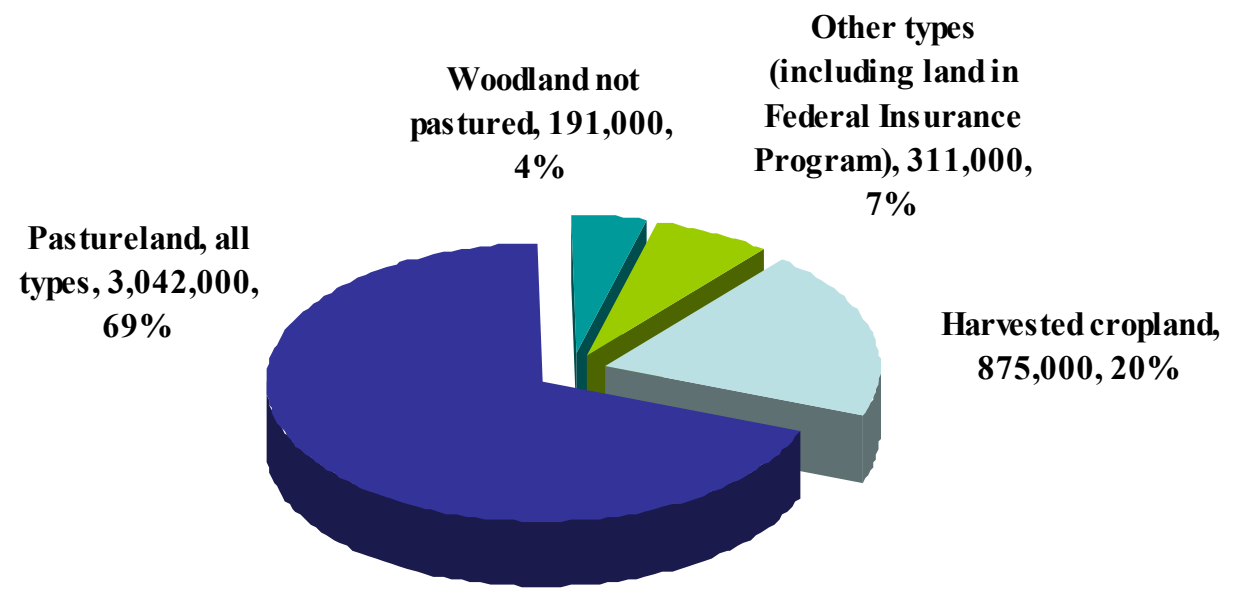

Figure 2. Land use in the central region of Florida by acre and percent of total

For cropland, Polk and Highlands Counties, with 190,000 and 169,000 acres of cropland respectively, ranked first and second, followed by DeSoto, Hardee, Hillsborough, Indian River, Manatee, Okeechobee, and St. Lucie Counties, each with more than 100,000 acres of cropland. For harvested cropland, Polk County ranked first in the region, with over 134,000 acres, followed by Highlands, and St. Lucie Counties, with more than 99,000 and 98,000 acres, respectively. Osceola was the leading county for land used for pasture, with more than 533,000 acres, followed by Highlands, and Polk Counties, each with more than 400,000 acres.

Orange was the largest county for nursery, greenhouse, and floriculture production, with more than 32 million square feet under glass or other protection (33 percent of the regional total), while Highlands and Osceola Counties had the highest nursery, greenhouse, and floriculture production in the open, each with more than 9,000 acres (each representing 15 percent of regional total) in 2002. 
For the central region as a whole, reported cropland acreage increased by 8 percent, while harvested cropland decreasaed by 7 percent. The pastureland and rangeland acreage decreased by 19 percent during the 1997-2002 period. There was, however, significant variation across the region. Reported changes in cropland acreage ranged from an 83 percent increase in Sarasota County to a 48 percent decrease in Seminole County. Overall, 9 counties reported cropland acreage increases by a larger percentage than the regional average, and 8 counties reflected a decrease between the two census periods. In addition to Seminole County, significant increases in cropland acreage were reported for Highlands, Okeechobee, and Osceola Counties.

\section{Market Value of Agricultural Products Sold, Production Expenses, and Net Farm Cash Income from Operation}

Table 4 reports the market value of agricultural products sold in the central region of Florida. According to the 2002 Census of Agriculture, "market value of agricultural products sold represents the gross market value before taxes and production expenses of all agricultural products sold or removed from the place in 2002 regardless of who received the payment." Market value is equivalent to total sales, including the value of any shares received by partners, landlords, contractors, or others associated with the operation, and the value of commodities placed in Commodity Credit Corporation (CCC) loans. The market value of agricultural products sold does not include payments received for participation in other federal farm programs nor income from farm-related sources such as custom-work, other agricultural services, or income from non-farm sources.

The market value of agricultural products sold in the central region of Florida exceeded $\$ 2.6$ billion in 2002 (Table 4), averaging over $\$ 135,000$ per farm. Average value varied dramatically from $\$ 315,000$ in Manatee County (the highest) to $\$ 15,000$ in Citrus County (the lowest). Total farm production expenses exceeded $\$ 2$ billion, averaging over $\$ 108,000$ per farm. Net farm cash income from operations in 2002 exceeded $\$ 641$ million for all farms in the region. Net farm cash income from operations was negative for Sumter County and less than $\$ 500$ for Citrus and Pinellas Counties. Across the region, market value of products sold decreased by 3 percent between the two census periods, but increases in the total market values were reported in 10 of the 20 counties in the region.

Hillsborough County registered the highest market value of agricultural products sold in the central region, with more than $\$ 392$ million (15 percent of regional total), and the highest net farm cash income from operations, with $\$ 107$ million (17 percent of regional total). Polk County was second behind Hillsborough in the market value of agricultural products sold, while Lake County was the second in the net farm cash income from operations.

\section{Value of Agricultural Products by Commodity Group}

For the central region of Florida, the value of fruits, tree nuts, and berries constituted the highest portion of the market value of agricultural products in 2002, amounting to almost $\$ 1.1$ billion, or 41 percent of the regional total (Table 5). The value of nursery, greenhouse, floriculture, and sod was the second highest with about $\$ 723$ million, or 27 percent of the regional total (Figure 3). The value of the other vegetables, melons, potatoes, and sweet potatoes ranked third with almost $\$ 271$ million, 
or 10 percent. The market value of the other livestock (except milk and dairy products) along with aquaculture exceeded $\$ 353$ million, or 13 percent of the total. Hillsborough County registered the highest market value of agricultural products, exceeding $\$ 333$ million (15 percent of the region). Polk County had the highest value for fruits, tree nuts, and berries ( $\$ 217$ million or 20 percent of the region), while Orange County had the highest value for nursery, greenhouse, floriculture, and sod products (more than $\$ 217$ million, or 30 percent of the value of these products in the region).

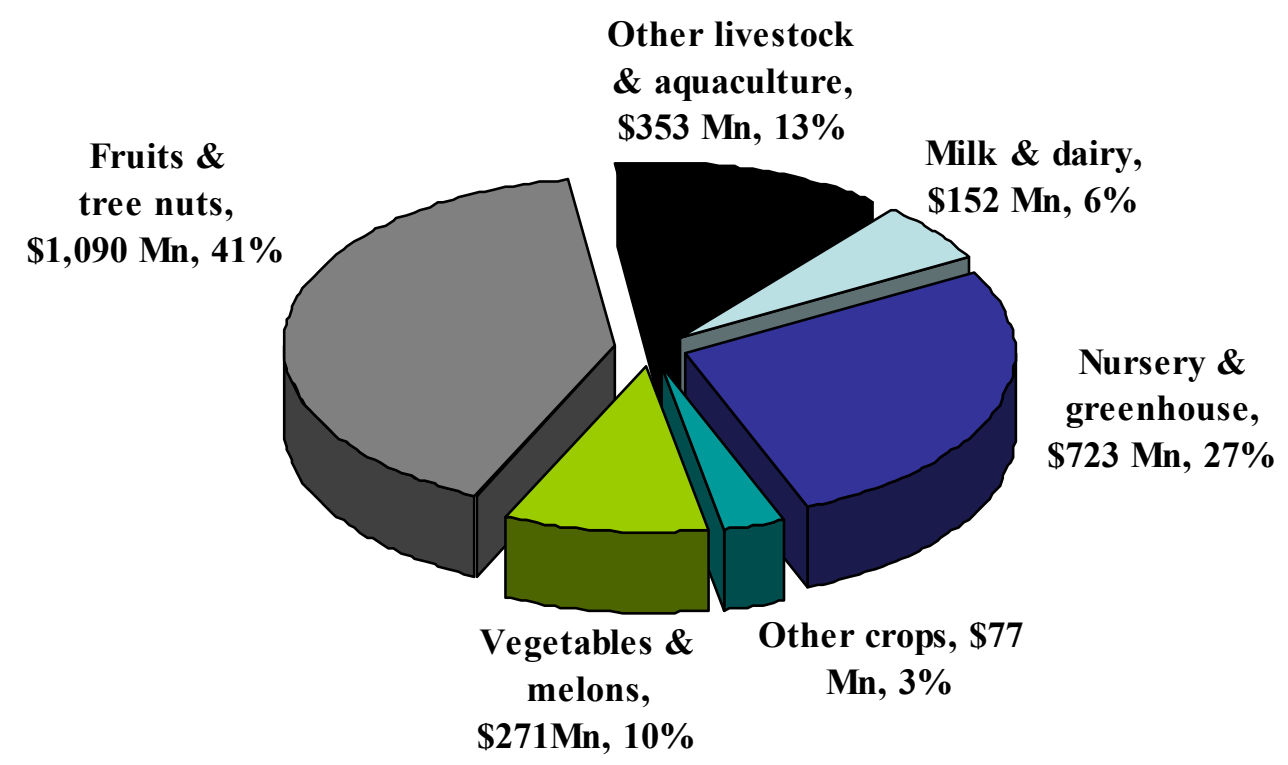

Figure 3. Market value of agricultural products sold in 2002 by commodity group in central region of Florida

Trends during 1997-2002 showed a 9 percent decrease in the value of livestock, poultry, and their products for the region. Within this broader group, the value of poultry decreased by 43 percent, milk and dairy products decreased by 19 percent, while cattle and calves' increased by 4 percent. The value of fruits, tree nuts, and berries (the major crop in the central region) increased by 5 percent during 1997-2002; however, the extent of change in most counties was far from average and ranged from an increase of 63 percent in Hernando County to a decrease of 36 percent in Osceola County.

\section{Principal Operator, Business Organization, and Ethnicity}

In 2002, there were more than 28,000 farm operators in the central region (31 percent were women). Farming was indicated as a primary occupation by 52 percent of all farm operators (Table 6). Hillsborough County, with more than 4,400 farm operators, accounted for 24 percent (the highest number of farm operators in the central region). The number of women operators in Hillsborough County was also the highest in the region.

Trends during 1997-2002 showed that the number of farmers who reported farming as the primary occupation in the central region increased by 16 percent. During the same time period, the number of farms with farming as the primary occupation in Okeechobee, De Soto, and Highlands Counties increased by 49,47 , and 46 percent, respectively. 
With respect to the type of business organization, most farms (81 percent) in the central region were family and individual types of businesses (Table 7). Partnership farms, corporate farms (family or other than family held), cooperatives, estates or trusts, and institutional farms together comprised the remaining 19 percent of the total number of farms (Figure 4). While the number of all corporate farms (family and non-family held) was only 11 percent of all farms in the region, they represented 37 percent of farm acreage. Also, partnership farms, with 6 percent of all farms, had more than 20 percent of the farm acreage, whereas the family and individual farms accounted for 42 percent of all the farm acreage in the region (Figure 4).

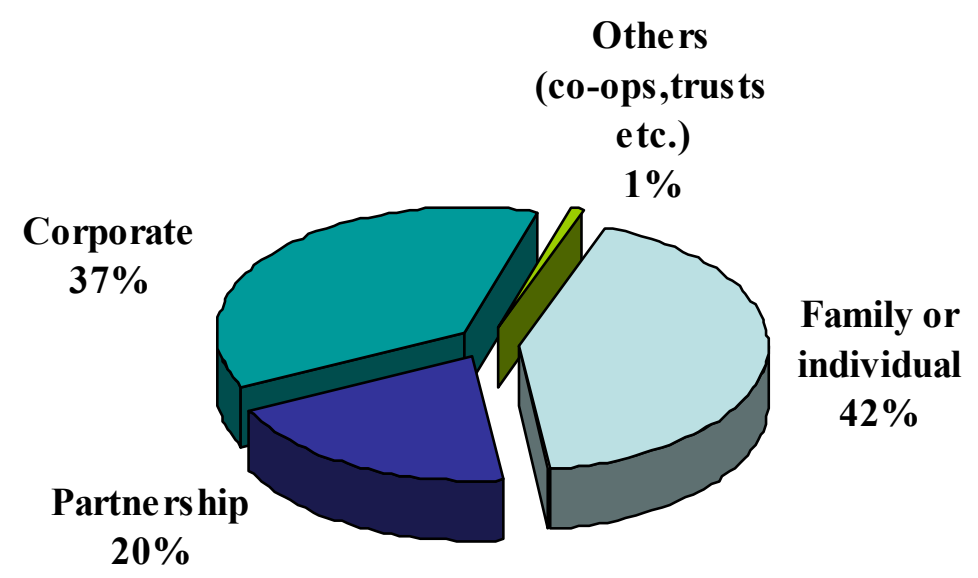

Figure 4. Farm acreage percentages by type of business organization in central region of Florida

The white ethnic group operated most of the farms in the central region. Other ethnic groups identified as farm operators included Black or African American, Spanish, Hispanic or Latino Origin, American Indian, Alaska Native, Native Hawaiian, other Pacific Islander, and Asian. Whites accounted for 93 percent of all farm operators and operated 97 percent of all farm acreage in the region (Table 8). Hillsborough County had the highest number of minority ethnic group farms, farm operators and acreage.

\section{Economic Impact Results}

As noted earlier, the IMPLAN software package and associated databases allow the estimation of multipliers to capture the total economic impact of agricultural and natural resource industries within a regional or local economy. This section reports the results of impact estimations for counties in the central region for each of the agricultural sectors within IMPLAN. Again estimates are based only on the IMPLAN data set with no attempt to adjust for particular local situations and the focus is only on impacts of sales and expenditures associated with farm operations and related manufacturing. ${ }^{3}$

For each reported measure (output, value-added and employment) for each county, the impacts reported include the direct output of the agricultural sector in question, impacts associated with indirect inter-industry purchases of inputs by agriculture and its supplying industries, and the

3 Readers desiring more information on IMPLAN should consult Mulkey \& Hodges, "Using Implan to Assess Local Economic Impacts," Food and Resource Economics Department, UF/IFAS, 2001, http//edis.ifas.ufl.edu/FE168. 
induced impacts of spending by employees and owners of agricultural and supplying industries. Reported impacts include activities within production agriculture, natural resource industry (forestry, mining, and fishing), food and forest products manufacturing, agricultural input supply, and agricultural services. Impacts are also reported in terms of labor income and indirect business taxes.

\section{Output Impacts}

Table 9 presents estimates of output impacts for agriculture and natural resource industries by sector and by county for the central region of Florida. The total output impacts for all counties in the central region exceeded $\$ 27$ billion. Fruit and vegetable farming and processing was the largest sector, contributing over $\$ 7.6$ billion in total output impacts and accounting for 28 percent of the total in the region. The agricultural inputs and services sector with more than $\$ 6.3$ billion in output impacts is the next largest sector in the region. Other food products manufacturing with close to $\$ 4$ billion; environmental horticulture with more than $\$ 3$ billion; and forestry, wood and paper products manufacturing with $\$ 2.4$ billion in output impacts were also major contributors to the output impacts of agriculture and natural resource industries in the region.

In total output impacts, Hillsborough and Polk Counties with more than $\$ 6.7$ billion and $\$ 6.6$ billion in output impacts, respectively, were the largest, followed by Orange and Manatee Counties with $\$ 3.1$ and $\$ 2.6$ billion. The agricultural inputs and services sector in Polk County with $\$ 2.7$ billion accounted for 43 percent of total output impacts for this sector in the region and 40 percent of the total output impacts for the county. Orange County had the highest output impact in environmental horticulture, with a total of $\$ 802$ million, or 26 percent of the total for the region. Hillsborough County had more than $\$ 430$ million in output impacts in the fishing and seafood products sector, or 60 percent of the total for this sector in the region. Manatee County had the highest output impacts in fruit and vegetable farming and processing accounting, with 29 percent of the total for this sector in the region and 84 percent of the total output impacts for the county.

\section{Employment Impacts}

Employment impacts of agriculture and natural resource industries by county and industry group are reported in Table 10. More than 306,000 full- and part-time jobs were generated by agricultural and natural resource industries in the central region of Florida. The agricultural inputs and services sector (the dominant industry group) produced over 91,000 jobs, accounting for 30 percent of total employment impacts for all industry groups in the region. The fruit and vegetable farming and processing industry and the environmental horticulture industry produced employment impacts of more than 75,000 and 56,000 jobs, accounting for 25 and 18 percent of employment impacts for the region, respectively (Figure 5). These three industry groups accounted for 73 percent of all employment impacts in the region. The other food manufacturing industry produced 26,000 jobs.

Polk and Hillsborough Counties had the dominant position in employment impacts, with more than 67,000 and 63,000 jobs, respectively, accounting for 22 and 21percent of the total for the region. Orange County with a total of more than 32,000 jobs reflected a strong employment impacts in the other food manufacturing sector $(6,500$ jobs $)$, accounting for 25 percent of the total for the region. 


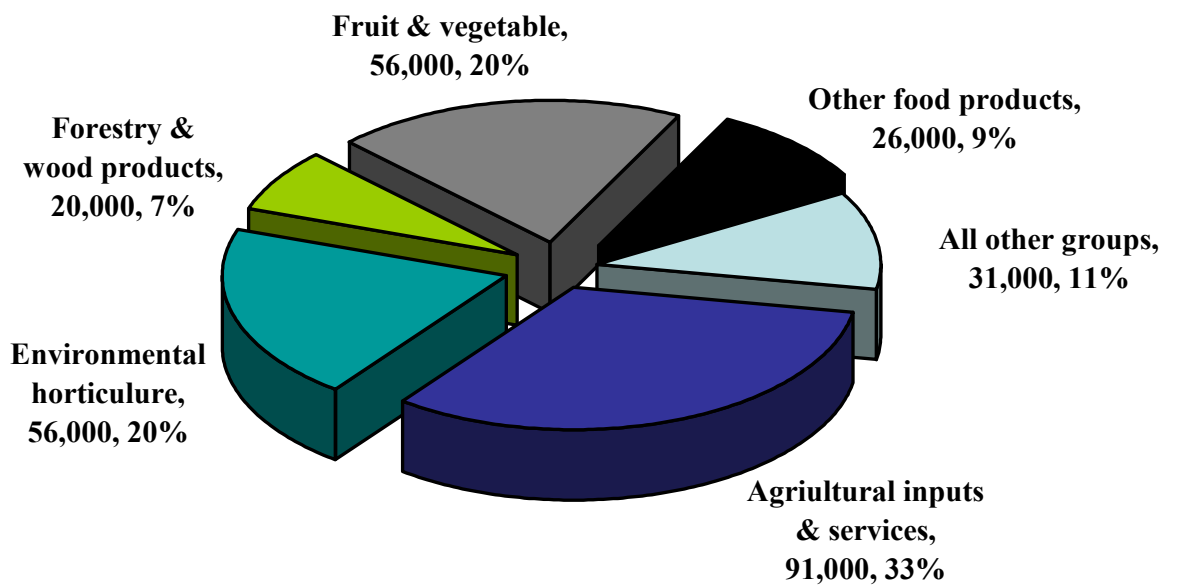

Figure 5. Employment impacts by industry group and the number of jobs in central region of Florida

\section{Value-added Impacts}

Value-added is the market value of a firm's output less the value of the inputs which it has purchased from others. It can be viewed as a net economic contribution by the industry sector after direct costs have been subtracted from gross sales. Value-added is the difference between the value of goods as they leave a particular stage of production and the costs of the goods as they entered that stage. Value-added includes payments made by industries to workers, interest, profits, and indirect business taxes. The total value-added impacts of agriculture and natural resource industries in the central region exceeded $\$ 13.6$ billion (Table 11). Fruit and vegetable farming and processing with $\$ 4$ billion in value-added impacts (the highest in the region) accounted for over 29 percent of total value-added impacts for the region. Agricultural inputs and services with $\$ 2.8$ billion was another major contributor to total value-added impacts. Environmental horticulture (greenhouse/nursery production and landscaping services) generated value-added impacts of over $\$ 2$ billion (Figure 6).

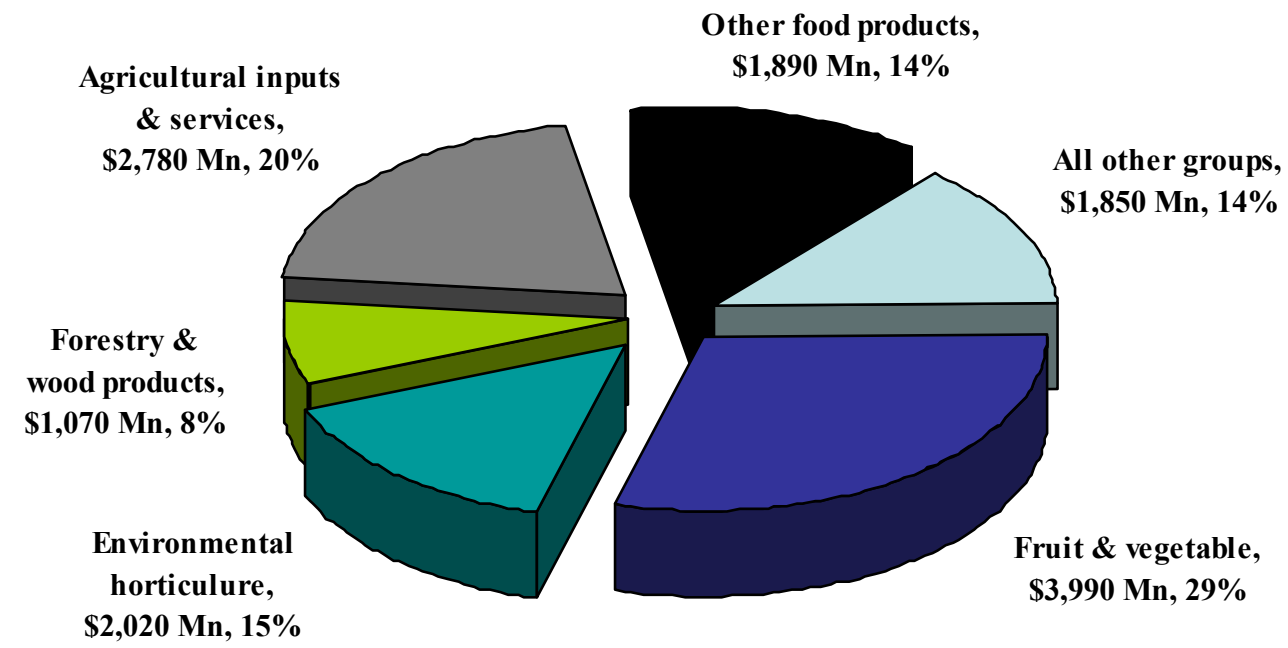

Figure 6. Value-added impacts by industry group in central region of Florida 
Hillsborough County with $\$ 3.2$ billion accounted for over 24 percent of the value-added impacts, and Polk County with $\$ 3.1$ billion accounted for 23 percent of the total value-added impacts for all counties in the region. Hillsborough County's dominant position was obvious in most sectors of the agricultural and natural resource industries; however, Polk County with more than $\$ 1$ billion in the agricultural inputs and services sector had the highest impact for the region. Polk County had also the highest value-added impacts in the mining sector, with $\$ 382$ million, or 60 percent of the total for the region. Manatee County's value-added impact of $\$ 1.2$ billion in the fruit and vegetable farming and processing sector was the highest in the region, accounting for 30 percent of the total.

\section{Labor Income Impacts}

Labor income impacts totaled over $\$ 8.8$ billion (Table 12). More than 25 percent of the total labor income impact was due to activities in fruit and vegetables farming and processing ( $\$ 2.2$ billion). Other major contributors included the agricultural inputs and services sector ( $\$ 2.2$ billion), environmental horticulture with $\$ 1.4$ billion, and the other food product manufacturing sector with $\$ 1.1$ billion in labor income impacts.

For labor income impacts, Hillsborough and Polk Counties were the largest, with more than \$2.1 billion and $\$ 2$ billion, respectively, accounting for 24 and 20 percent of the regional total. Labor income impacts of $\$ 629$ million in Manatee County for the fruit and vegetables farming and processing, and \$542 million in Orange County for environmental horticulture ranked first in the region.

\section{Indirect Business Tax Impacts}

Indirect business taxes consist of excise taxes, property taxes, fees, licenses, and sales taxes paid by businesses. These taxes occur during the normal operation of businesses but do not include taxes on profit or income. Total indirect business tax impacts were more than $\$ 857$ million for the region (Table13). Like most of the other economic impact measures, the fruit and vegetable farming and processing sector with a total of $\$ 239$ million indirect business tax impacts topped the same value for the other sectors in the region. For indirect business tax impacts, Polk County showed its dominant position in the central region with $\$ 220$ million, or 26 percent. Hillsborough County had $\$ 194$ million in total indirect business tax impacts, accounting for over 22 percent of this value for the whole central region in Florida. Orange County showed the next highest indirect business tax impacts in the region with $\$ 82$ million.

\section{Acknowledgement}

Constructive comments given by reviewer, Dr. John J. Haydu, are greatly appreciated 
Table 1. Farms by North American Industry Classification System, central region of Florida, 2002

\begin{tabular}{|c|c|c|c|c|c|c|c|c|c|c|c|c|c|}
\hline County & $\begin{array}{l}\begin{array}{c}\text { Total } \\
\text { farms }\end{array}\end{array}$ & $\begin{array}{l}\text { Oilseed } \\
\& \text { grain } \\
\text { farming } \\
(1111)\end{array}$ & $\begin{array}{l}\text { Vegetable } \\
\text { \& melon } \\
\text { farming } \\
(11121)\end{array}$ & $\begin{array}{c}\text { Fruit \& } \\
\text { tree nut } \\
\text { farming } \\
(1113)\end{array}$ & $\begin{array}{c}\text { Greenhouse, } \\
\text { nursery, \& } \\
\text { floriculture } \\
(1114)\end{array}$ & $\begin{array}{c}\text { Other } \\
\text { crop } \\
\text { farming } \\
(1119)\end{array}$ & $\begin{array}{c}\text { Sugarcane, hay, } \\
\& \text { other crop } \\
(11193,11194 \\
11199)\end{array}$ & $\begin{array}{c}\text { Beef cattle } \\
\text { ranching \& } \\
\text { farming } \\
(11211)\end{array}$ & $\begin{array}{l}\text { Dairy cattle } \\
\& \text { milk } \\
\text { production } \\
(11212)\end{array}$ & $\begin{array}{c}\text { Hog \& pig } \\
\text { farming } \\
(1122)\end{array}$ & $\begin{array}{c}\text { Poultry } \\
\text { \& egg } \\
\text { production } \\
(1123)\end{array}$ & $\begin{array}{c}\text { Sheep } \\
\text { \& goat } \\
\text { farming } \\
(1124)\end{array}$ & $\begin{array}{c}\text { Animal } \\
\text { aquaculture \& } \\
\text { other animal } \\
\text { production } \\
(1125,1129)\end{array}$ \\
\hline Brevard & 555 & 1 & 4 & 225 & 64 & 8 & 8 & 117 & - & 2 & 5 & 7 & 122 \\
\hline Citrus & 432 & - & 4 & 27 & 28 & 9 & 9 & 196 & 1 & 10 & 8 & 13 & 136 \\
\hline DeSoto & 1,153 & 2 & 6 & 594 & 28 & 16 & 16 & 410 & 12 & 5 & 6 & 4 & 70 \\
\hline Hardee & 1,142 & - & 8 & 598 & 40 & 11 & 11 & 416 & 5 & 4 & 1 & 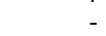 & 59 \\
\hline Hernando & 617 & 1 & 10 & 55 & 39 & 37 & 37 & 298 & 6 & 6 & 12 & 8 & 145 \\
\hline Highlands & 1,035 & - & 7 & 368 & 80 & 6 & 6 & 447 & 23 & 28 & 6 & 13 & 57 \\
\hline Hillsborough & 2,969 & 3 & 85 & 799 & 254 & 47 & 47 & 1,146 & 42 & 33 & 25 & 40 & 495 \\
\hline Indian River & 480 & - & 8 & 250 & 37 & 3 & 3 & 81 & - & 3 & 7 & 2 & 89 \\
\hline Lake & 1,798 & 4 & 27 & 569 & 279 & 50 & 50 & 567 & 8 & 13 & 9 & 15 & 257 \\
\hline Manatee & 852 & 2 & 39 & 151 & 110 & 7 & 7 & 391 & 11 & 10 & 2 & - & 129 \\
\hline Okeechobee & 638 & - & 10 & 45 & 29 & 9 & 9 & 456 & 30 & - & 2 & 3 & 54 \\
\hline Orange & 901 & - & 1 & 247 & 312 & 12 & 12 & 164 & 6 & 1 & 12 & 5 & 141 \\
\hline Osceola & 519 & - & 3 & 116 & 58 & 7 & 7 & 239 & - & 5 & 5 & 6 & 80 \\
\hline Pasco & 1,222 & 1 & 9 & 291 & 70 & 38 & 38 & 533 & 19 & 17 & 27 & 20 & 197 \\
\hline Pinellas & 111 & - & 2 & 12 & 62 & 3 & 3 & 6 & - & - & 4 & 1 & 21 \\
\hline Polk & 3,114 & 10 & 23 & 1,732 & 137 & 21 & 21 & 904 & 13 & 16 & 15 & 21 & 222 \\
\hline St. Lucie & 477 & - & 4 & 245 & 35 & - & - & 111 & 2 & 11 & 1 & 11 & 57 \\
\hline Sarasota & 371 & - & 3 & 38 & 54 & 6 & 6 & 165 & - & 1 & 8 & 3 & 93 \\
\hline Seminole & 376 & - & 1 & 90 & 71 & 3 & 3 & 87 & 3 & 4 & 8 & 7 & 102 \\
\hline Sumter & 902 & 4 & 24 & 13 & 29 & 45 & 45 & 630 & 24 & 9 & 5 & 7 & 112 \\
\hline Total & 19,664 & 28 & 278 & 6,465 & 1,816 & 338 & 338 & 7,364 & 205 & 178 & 168 & 186 & 2,638 \\
\hline
\end{tabular}


Table 2. Farm size distribution, and investment in land \& building, machinery, central region of Florida, 2002, and percent change, 1997-2002

\begin{tabular}{|c|c|c|c|c|c|c|c|c|c|c|c|c|c|c|c|c|c|}
\hline \multirow[b]{2}{*}{ County } & \multicolumn{4}{|c|}{ Farms } & \multicolumn{2}{|c|}{$\begin{array}{c}\text { Average } \\
\text { farm size }\end{array}$} & \multicolumn{2}{|c|}{$\begin{array}{c}\text { Farms less than } \\
100 \text { acres }\end{array}$} & \multicolumn{2}{|c|}{$\begin{array}{l}\text { Farms } 100 \text { to } \\
499 \text { acres }\end{array}$} & \multicolumn{2}{|c|}{$\begin{array}{l}\text { Farms } 500 \text { acres } \\
\text { or more }\end{array}$} & \multicolumn{4}{|c|}{ Estimated asset value of land and buildings } & \multirow{2}{*}{ 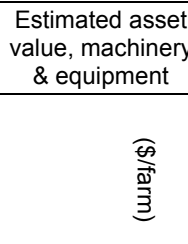 } \\
\hline & 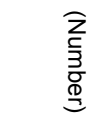 & $\begin{array}{l}\widehat{\circ} \\
\text { 宅 }\end{array}$ & 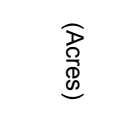 & $\begin{array}{l}\text { å } \\
\text { 高 }\end{array}$ & 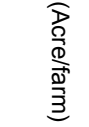 & $\begin{array}{l}\widehat{\circ} \\
\stackrel{9}{0} \\
0\end{array}$ & 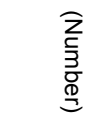 & 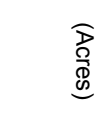 & 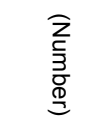 & 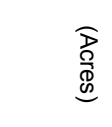 & 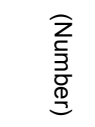 & 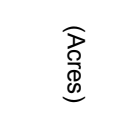 & $\begin{array}{l}\bar{\theta} \\
\overline{\mathrm{O}} \\
\overline{0}\end{array}$ & 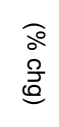 & 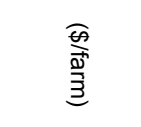 & 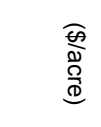 & \\
\hline Brevard & 555 & -9 & 187,570 & -33 & 338 & -27 & 460 & 8,756 & 53 & 10,200 & 42 & 168,614 & 437,722 & -1 & 783,045 & 2,385 & 42,237 \\
\hline Citrus & 432 & 5 & 47,209 & -6 & 109 & -10 & 351 & 8,400 & 68 & 14,000 & 13 & 24,809 & 133,781 & 19 & 309,677 & 2,498 & 28,773 \\
\hline De Soto & 1,153 & 23 & 388,177 & 20 & 337 & -2 & 892 & 21,882 & 151 & 30,742 & 110 & 335,553 & 905,985 & 9 & 786,445 & 2,415 & 38,912 \\
\hline Hardee & 1,142 & -12 & 346,191 & 0 & 303 & 13 & 731 & 21,317 & 279 & 61,938 & 132 & 262,936 & 819,371 & -11 & 718,746 & 2,341 & 40,190 \\
\hline Hernando & 617 & -4 & 65,315 & 17 & 106 & 23 & 507 & 12,548 & 82 & 18,618 & 28 & 34,149 & 264,996 & 21 & 429,492 & 5,093 & 51,371 \\
\hline Highlands & 1,035 & 1 & 576,900 & 18 & 557 & 16 & 715 & 18,585 & 188 & 44,720 & 132 & 513,595 & $1,246,204$ & 30 & $1,206,393$ & 2,256 & 48,988 \\
\hline Hillsborough & 2,969 & -19 & 284,910 & 8 & 96 & 33 & 2,617 & 52,131 & 257 & 49,233 & 95 & 183,546 & $1,350,058$ & 8 & 454,871 & 5,410 & 42,898 \\
\hline Indian River & 480 & -8 & 191,333 & 12 & 399 & 23 & 351 & 6,860 & 70 & 16,491 & 59 & 167,043 & 550,135 & -6 & $1,146,115$ & 2,969 & 41,314 \\
\hline Lake & 1,798 & -1 & 180,245 & -7 & 100 & -6 & 1,558 & 34,475 & 175 & 37,021 & 65 & 108,749 & 803,802 & 32 & 447,801 & 4,290 & 26,616 \\
\hline Manatee & 852 & -5 & 301,231 & 12 & 354 & 18 & 557 & 13,906 & 176 & 37,620 & 119 & 249,705 & 926,020 & 35 & $1,086,878$ & 3,142 & 50,879 \\
\hline Okeechobee & 638 & 1 & 392,495 & 1 & 615 & 0 & 350 & 9,032 & 145 & 34,068 & 143 & 349,395 & 802,538 & 33 & $1,263,840$ & 2,037 & 32,721 \\
\hline Orange & 901 & -15 & 146,637 & -17 & 163 & -1 & 781 & 14,670 & 93 & 17,721 & 27 & 114,246 & 571,809 & 3 & 634,638 & 3,931 & 49,786 \\
\hline Osceola & 519 & -19 & 652,673 & 6 & 1,258 & 31 & 368 & 7,685 & 75 & 17,881 & 76 & 627,107 & $1,075,015$ & 32 & $2,067,337$ & 1,690 & 72,904 \\
\hline Pasco & 1,222 & -9 & 168,716 & 0 & 138 & 10 & 1,018 & 21,628 & 152 & 28,879 & 52 & 118,209 & 697,777 & 25 & 570,545 & 3,863 & 22,407 \\
\hline Pinellas & 111 & -33 & 1,589 & -44 & 14 & -18 & 105 & 505 & 6 & 808 & 0 & 0 & 39,888 & -7 & 362,615 & 31,732 & 32,118 \\
\hline Polk & 3,114 & -1 & 626,634 & 0 & 201 & 2 & 2,419 & 60,201 & 508 & 105,593 & 187 & 460,840 & $1,835,381$ & 29 & 588,641 & 2,899 & 36,440 \\
\hline St. Lucie & 477 & -22 & 221,537 & -4 & 464 & 23 & 303 & 7,778 & 109 & 24,266 & 65 & 189,493 & 742,933 & 22 & $1,564,070$ & 3,239 & 58,765 \\
\hline Sarasota & 371 & -20 & 121,310 & -6 & 327 & 17 & 295 & 5,316 & 38 & 8,166 & 38 & 107,034 & 312,242 & -1 & 841,623 & 2,995 & 22,024 \\
\hline Seminole & 376 & -21 & 27,987 & -32 & 74 & -15 & 345 & 5,460 & 27 & 4,327 & 4 & 0 & 160,155 & 16 & 424,814 & 6,137 & 14,884 \\
\hline Sumter & 902 & -11 & 187,373 & -1 & 208 & 11 & 670 & 18,827 & 188 & 38,670 & 44 & 129,876 & 454,352 & 23 & 502,601 & 2,405 & 23,327 \\
\hline Total & 19,664 & -8 & $5,116,032$ & 2 & 308 & 11 & 15,393 & 349,962 & 2,840 & 600,962 & 1,431 & $4,144,899$ & $14,130,164$ & 17 & 809,509 & 4,686 & 38,878 \\
\hline
\end{tabular}

Source: 2002 Census of Agriculture, Florida state and county data, Volume 1, Geographic area series, Part 9, AC-02-A-9, Issued June 2004, National Statistics Service, US Department of Agriculture 
Table 3A. Farmland use, central region of Florida, 2002, and percent change, 1997-2002

\begin{tabular}{|c|c|c|c|c|c|c|c|c|c|}
\hline \multirow{2}{*}{ County } & \multicolumn{2}{|c|}{ All cropland } & \multicolumn{2}{|c|}{ Harvested cropland } & \multicolumn{2}{|c|}{$\begin{array}{l}\text { Cropland used only } \\
\text { for pasture or grazing }\end{array}$} & \multirow{2}{*}{$\begin{array}{l}\text { Cropland in cultivated } \\
\text { summer fallow } \\
\text { (Acres) }\end{array}$} & \multirow{2}{*}{$\begin{array}{c}\begin{array}{c}\text { Nursery, greenhouse, } \\
\text { floriculture (1), under glass } \\
\text { or other protection }\end{array} \\
\text { (Square feet) }\end{array}$} & \multirow{2}{*}{$\begin{array}{c}\begin{array}{c}\text { Nursery, greenhouse, } \\
\text { floriculture (1), in the open }\end{array} \\
\text { (Acres) }\end{array}$} \\
\hline & (Acres) & (\% chg) & (Acres) & (\% chg) & (Acres) & (\% chg) & & & \\
\hline Brevard & 23,106 & -16 & 15,651 & -30 & 2,835 & 89 & & 491,312 & 3,195 \\
\hline Citrus & 12,331 & -38 & 4,051 & -40 & 343 & -13 & & 309,360 & 67 \\
\hline De Soto & 115,356 & -8 & 87,005 & -3 & 5,127 & -60 & 164 & 565,944 & 5,297 \\
\hline Hardee & 115,676 & 10 & 70,728 & 4 & & & & $8,311,588$ & 3,563 \\
\hline Hernando & 24,260 & 21 & 12,139 & 70 & & & & 92,443 & 471 \\
\hline Highlands & 168,996 & 42 & 99,362 & 6 & 921 & -75 & 67 & $6,406,413$ & 9,009 \\
\hline Hillsborough & 126,158 & 17 & 59,342 & -5 & 5,468 & -41 & 294 & $11,194,217$ & 5,241 \\
\hline Indian River & 102,916 & 19 & 65,311 & -15 & 3,746 & 303 & 36 & 139,676 & \\
\hline Lake & 73,958 & -8 & 35,899 & -14 & & & & $21,246,083$ & 4,225 \\
\hline Manatee & 117,173 & 14 & 60,900 & -6 & 5,508 & -16 & & $6,625,064$ & 4,903 \\
\hline Okeechobee & 115,292 & 68 & 38,984 & 8 & 1,565 & 28 & & 51,676 & 2,125 \\
\hline Orange & 25,489 & -42 & 16,904 & -45 & 3,512 & -48 & & $32,728,420$ & 4,393 \\
\hline Osceola & 76,148 & 50 & 27,448 & -9 & 1,166 & -27 & & 702,610 & 9,108 \\
\hline Pasco & 52,943 & -8 & 21,332 & -17 & & & & $1,469,737$ & 1,522 \\
\hline Pinellas & & & 319 & -47 & & & & 905,228 & 89 \\
\hline Polk & 189,970 & 2 & 134,101 & 2 & 7,206 & -14 & & $4,816,271$ & 3,043 \\
\hline St. Lucie & 118,847 & -13 & 97,929 & -20 & 748 & -78 & & $1,707,134$ & 517 \\
\hline Sarasota & 33,362 & 83 & 6,418 & 18 & 44 & & & 357,106 & 1,314 \\
\hline Seminole & 3,763 & -48 & 2,564 & -41 & & & & $1,296,180$ & 813 \\
\hline Sumter & 44,950 & -17 & 18,131 & 4 & 560 & & 89 & 190,570 & 722 \\
\hline Total & $1,540,694$ & 8 & 874,518 & -7 & 38,749 & -32 & 650 & $99,607,032$ & 59,617 \\
\hline
\end{tabular}

Source: 2002 Census of Agriculture, Florida state and county data, Volume 1, Geographic area series, Part 9, AC-02-A-9, Issued June 2004, National Statistics Service, US Department of Agriculture 
Table 3B. Farmland use, central region of Florida, 2002, and percent change, 1997-2002

\begin{tabular}{|c|c|c|c|c|c|c|c|c|c|c|c|c|c|c|}
\hline \multirow{2}{*}{ County } & \multicolumn{2}{|c|}{$\begin{array}{l}\text { Pasture- and } \\
\text { rangeland, other } \\
\text { than cropland \& } \\
\text { woodland pastured }\end{array}$} & \multicolumn{2}{|c|}{ Pastureland, all types } & \multicolumn{2}{|c|}{ Woodland } & \multicolumn{2}{|c|}{$\begin{array}{l}\text { Woodland } \\
\text { pastured }\end{array}$} & \multicolumn{2}{|c|}{$\begin{array}{l}\text { Woodland } \\
\text { not pastured }\end{array}$} & \multicolumn{2}{|c|}{$\begin{array}{l}\text { Land in conservation } \\
\text { or wetlands reserve } \\
\text { programs }\end{array}$} & \multirow{2}{*}{ 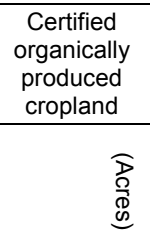 } & \multirow{2}{*}{ 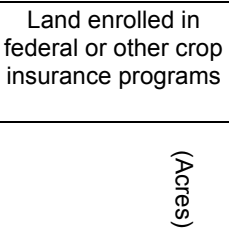 } \\
\hline & 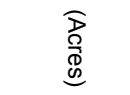 & $\begin{array}{l}\text { aे } \\
\text { ?a } \\
\text { ấ }\end{array}$ & 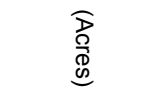 & $\begin{array}{l}\text { aे } \\
\text { @े } \\
\text { a }\end{array}$ & 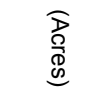 & $\begin{array}{l}\text { a } \\
\text { @) } \\
\text { ấ }\end{array}$ & 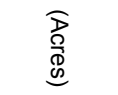 & $\begin{array}{l}\text { ò } \\
\text { ?a } \\
\text { â }\end{array}$ & 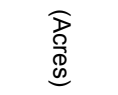 & $\begin{array}{l}\text { aे } \\
\text { @े } \\
\text { â }\end{array}$ & 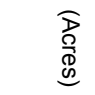 & $\begin{array}{l}\text { a } \\
\text { ̊े } \\
\text { a् }\end{array}$ & & \\
\hline Brevard & 90,039 & -56 & 143,849 & -40 & 57,518 & 41 & 49,190 & 41 & 8,328 & 46 & 94 & -57 & & 4,931 \\
\hline Citrus & 15,266 & -11 & 33,870 & -5 & 13,772 & 34 & 10,667 & 84 & 3,105 & -30 & & & & 2,313 \\
\hline $\begin{array}{l}\text { DeSoto } \\
\text { Hardee } \\
\text { Hernando }\end{array}$ & 201,221 & 28 & 256,342 & 34 & 36,676 & 127 & 31,897 & 193 & 4,779 & -9 & & & & 36,646 \\
\hline Highlands & 211,057 & -31 & 420,849 & 17 & 149,947 & 233 & 141,079 & 326 & 8,868 & -25 & & & & 54,213 \\
\hline Hillsborough & 92,497 & 3 & 171,303 & 6 & 33,131 & -28 & 17,458 & -52 & 15,673 & 64 & 540 & -80 & 235 & 21,305 \\
\hline $\begin{array}{l}\text { Indian River } \\
\text { Lake }\end{array}$ & 45,828 & 40 & 106,510 & 102 & 34,139 & -17 & 26,823 & 138 & 7,316 & -76 & & & 503 & 35,161 \\
\hline Manatee & 104,172 & -11 & 184,028 & 3 & 38,266 & 12 & 29,091 & 0 & 9,175 & 74 & (D) & & 28 & \\
\hline Okeechobee & 238,584 & -12 & 334,478 & 6 & 23,206 & -18 & 21,151 & 34 & 2,055 & -84 & 1,069 & 103 & & 18,235 \\
\hline Orange & 87,296 & 30 & 113,246 & -1 & 25,744 & -52 & 20,877 & -48 & 4,867 & -65 & & & 20 & 5,445 \\
\hline $\begin{array}{l}\text { Osceola } \\
\text { Pasco }\end{array}$ & 359,041 & -27 & 533,227 & -4 & 186,679 & 216 & 126,652 & 164 & 60,027 & 440 & 9 & & & 7,883 \\
\hline Pinellas & & & & & 131 & -55 & 55 & & 76 & & & & & 34 \\
\hline Polk & 303,046 & 4 & 411,959 & -3 & 78,191 & -32 & 60,250 & -31 & 17,941 & -33 & 1,216 & -8 & 394 & 48,862 \\
\hline St. Lucie & & -100 & 111,749 & 30 & & & & & 1,073 & -61 & 46 & -97 & 36 & 60,806 \\
\hline $\begin{array}{l}\text { Sarasota } \\
\text { Seminole }\end{array}$ & 50,831 & -41 & 107,470 & -8 & 33,732 & 59 & 29,739 & & 3,993 & & & & 39 & 851 \\
\hline Sumter & 62,463 & -31 & 113,315 & -20 & 68,472 & 110 & 24,593 & & 43,879 & & 158 & -65 & & 10,326 \\
\hline Total & $1,861,341$ & -19 & $3,042,195$ & 2 & 779,604 & 42 & 589,522 & 66 & 191,155 & 37 & 3,132 & -71 & 1,255 & 307,011 \\
\hline
\end{tabular}


Table 4. Market value of agricultural products sold, value of government payments, production expenses, and net farm cash income from operation, central region of Florida, 2002, and percent change, 1997-2002

\begin{tabular}{|c|c|c|c|c|c|c|c|c|c|}
\hline \multirow{2}{*}{ County } & \multicolumn{4}{|c|}{ Market value of all agricultural products sold } & \multirow{2}{*}{$\begin{array}{l}\begin{array}{c}\text { Value of government } \\
\text { payments }\end{array} \\
(\$ 1000)\end{array}$} & \multicolumn{2}{|c|}{ Total farm production expenses } & \multicolumn{2}{|c|}{ Net farm cash income from operation } \\
\hline & $(\$ 1000)$ & (\% chg) & (\$/farm) & (\% chg) & & $(\$ 1000)$ & (\$/farm) & $(\$ 1000)$ & (\$/farm) \\
\hline Brevard & 42,159 & 8 & 75,961 & 18 & 52 & 31,018 & 55,489 & 13,383 & 23,941 \\
\hline Citrus & 6,606 & 1 & 15,293 & -3 & 57 & 7,609 & 17,614 & 450 & 1,042 \\
\hline DeSoto & 180,096 & -1 & 156,198 & -19 & & 138,594 & 120,307 & 44,586 & 38,703 \\
\hline Hardee & 166,203 & 7 & 145,537 & 21 & 230 & 125,496 & 110,084 & 41,582 & 36,476 \\
\hline Hernando & 21,708 & -10 & 35,183 & -5 & 128 & 20,380 & 33,031 & 3,010 & 4,878 \\
\hline Highlands & 236,005 & 16 & 228,024 & 14 & 135 & 191,890 & 185,760 & 47,131 & 45,625 \\
\hline Hillsborough & 392,432 & 12 & 132,176 & 39 & 527 & 286,542 & 96,544 & 107,406 & 36,188 \\
\hline Indian River & 116,913 & 20 & 243,569 & 31 & 78 & 89,860 & 187,209 & 28,681 & 59,752 \\
\hline Lake & 178,076 & 4 & 99,041 & 5 & 103 & 98,308 & 54,768 & 75,401 & 42,006 \\
\hline Manatee & 268,480 & 11 & 315,118 & 18 & 112 & 210,921 & 247,559 & 62,114 & 72,903 \\
\hline Okeechobee & 144,376 & 5 & 226,295 & 4 & 405 & 125,477 & 197,602 & 22,223 & 34,997 \\
\hline Orange & 242,688 & -3 & 269,354 & 14 & 45 & 171,993 & 190,891 & 70,135 & 77,841 \\
\hline Osceola & 64,941 & -28 & 125,128 & -11 & 186 & 58,994 & 113,450 & 7,465 & 14,355 \\
\hline Pasco & 84,200 & -3 & 68,904 & 6 & 213 & 69,995 & 57,232 & 21,793 & 17,819 \\
\hline Pinellas & 7,960 & -36 & 71,710 & -4 & 18 & 7,847 & 71,332 & 236 & 2,149 \\
\hline Polk & 284,787 & 10 & 91,454 & 12 & 200 & 213,826 & 68,578 & 71,376 & 22,892 \\
\hline St. Lucie & 127,907 & -27 & 268,149 & -6 & 93 & 114,284 & 240,597 & 16,275 & 34,263 \\
\hline Sarasota & 17,801 & -28 & 47,982 & -11 & & 16,208 & 43,686 & 3,841 & 10,354 \\
\hline Seminole & 19,211 & -8 & 51,094 & 17 & & 14,357 & 38,083 & 6,187 & 16,412 \\
\hline Sumter & 30,644 & -14 & 33,974 & -3 & 326 & 34,874 & 38,577 & $-2,061$ & $-2,280$ \\
\hline Total & $2,633,193$ & -3 & 135,007 & 7 & 2,908 & $2,028,473$ & 108,420 & 641,214 & 29,516 \\
\hline
\end{tabular}


Table 5. Value of agricultural products by commodity group, central region of Florida, 2002, and percent change, 1997-2002

\begin{tabular}{|c|c|c|c|c|c|c|c|c|c|c|c|c|c|c|c|c|c|c|c|c|}
\hline \multirow[t]{2}{*}{ County } & \multicolumn{2}{|c|}{ 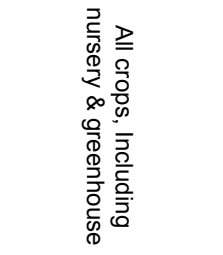 } & \multirow{2}{*}{ 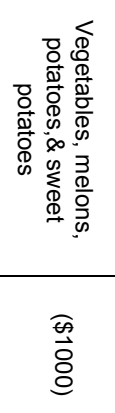 } & \multicolumn{2}{|l|}{ 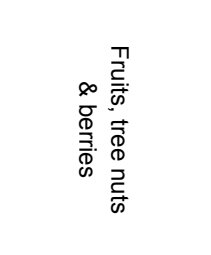 } & \multirow{2}{*}{ 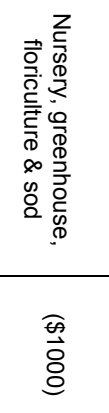 } & \multirow{2}{*}{ 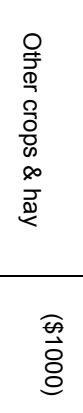 } & \multicolumn{2}{|c|}{ 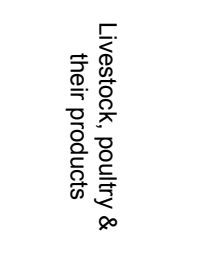 } & \multicolumn{2}{|c|}{ 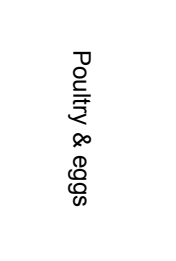 } & \multicolumn{2}{|l|}{ 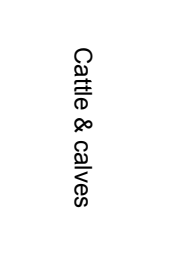 } & \multicolumn{2}{|c|}{ 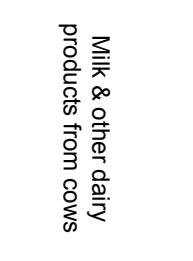 } & \multicolumn{2}{|c|}{ 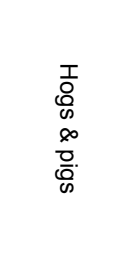 } & \multirow{2}{*}{ 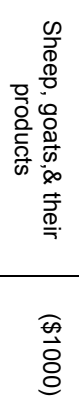 } & \multirow{2}{*}{ 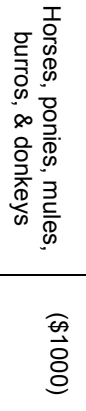 } & \multirow{2}{*}{ 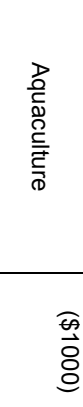 } \\
\hline & $\begin{array}{l}\overline{\theta A} \\
\vec{\circ} \\
\stackrel{\circ}{0}\end{array}$ & $\begin{array}{l}\widehat{\circ} \\
\text { 今a } \\
\text { a }\end{array}$ & & $\begin{array}{l}\widehat{\theta A} \\
\vec{\circ} \\
\stackrel{\circ}{0}\end{array}$ & $\begin{array}{l}\widehat{o} \\
\text { ô } \\
\text { âa }\end{array}$ & & & 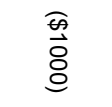 & $\begin{array}{l}\widehat{\circ} \\
\text { ⿳े } \\
\text { a }\end{array}$ & $\begin{array}{l}\widehat{\theta A} \\
\stackrel{\vec{\partial}}{\circ}\end{array}$ & $\begin{array}{l}\widehat{\circ} \\
\text { 产 } \\
\text { âd }\end{array}$ & $\begin{array}{l}\widehat{\theta A} \\
\vec{\circ} \\
\stackrel{0}{0}\end{array}$ & $\begin{array}{l}\widehat{o} \\
\text { ô } \\
\text { ôa }\end{array}$ & $\begin{array}{l}\widehat{\widehat{A}} \\
\vec{\circ} \\
\stackrel{0}{0}\end{array}$ & $\begin{array}{l}\text { aे } \\
\text { ̊े } \\
\text { âd }\end{array}$ & $\begin{array}{l}\widehat{\hat{\theta}} \\
\vec{\circ} \\
\stackrel{0}{0}\end{array}$ & $\begin{array}{l}\widehat{\circ} \\
\text { o } \\
\text { âd }\end{array}$ & & & \\
\hline Brevard & 35,306 & 7 & & 14,156 & 1 & 17,908 & 143 & 6,853 & 13 & 5 & -44 & 5,484 & 29 & & & 16 & -90 & 5 & 92 & 1,019 \\
\hline Citrus & 4,705 & 28 & 207 & 2,161 & & 2,224 & 105 & 1,901 & -33 & 11 & & 1,019 & -40 & & & 11 & -68 & 39 & 348 & \\
\hline DeSoto & 150,446 & -6 & 2,583 & 137,271 & -6 & 10,209 & & 29,650 & 29 & & & 18,637 & 45 & & & 1 & -98 & 4 & 203 & \\
\hline Hardee & 129,599 & 10 & 11,199 & 91,516 & -10 & 26,588 & & 36,604 & -3 & & & 15,958 & 7 & 19,797 & -6 & 11 & & 2 & & \\
\hline Hernando & 8,176 & 24 & & 1,621 & 63 & 5,822 & & 13,532 & -22 & & & & & 6,138 & 9 & 75 & -75 & 15 & 531 & 12 \\
\hline Highlands & 204,808 & 22 & & 154,975 & 11 & 48,847 & & 31,197 & -13 & & & 20,880 & 13 & 9,620 & -41 & 103 & -34 & 7 & 95 & \\
\hline Hillsborough & 333,745 & 23 & 73,916 & 143,053 & 2 & 115,794 & 941 & 58,687 & -26 & 13,344 & -35 & 11,530 & -10 & 9,735 & -16 & 167 & -77 & 74 & 1,293 & 21,006 \\
\hline Indian River & 110,225 & 21 & & 87,821 & 3 & & & 6,688 & 9 & 12 & & 4,903 & 46 & & & 5 & & 4 & & 1,462 \\
\hline Lake & 166,274 & 14 & 740 & 27,493 & 15 & 137,455 & 484 & 11,801 & -53 & & & 4,896 & 2 & 3,623 & -59 & 39 & -61 & & 553 & 37 \\
\hline Manatee & 247,023 & 12 & 158,557 & 49,188 & 10 & 39,012 & & 21,457 & 5 & 40 & & 9,154 & 16 & 9,959 & -13 & 28 & -65 & 11 & 548 & 949 \\
\hline Okeechobee & 36,677 & 11 & 13,073 & 19,626 & -3 & 3,848 & & 107,699 & 2 & 1 & -50 & 27,758 & 18 & 79,389 & -2 & & & & 142 & 140 \\
\hline Orange & 239,492 & -3 & & & & 220,167 & 151 & 3,195 & -14 & 42 & & 2,056 & -31 & 68 & & 12 & -84 & 7 & 253 & 274 \\
\hline Osceola & 43,953 & -28 & 5,130 & 19,051 & -36 & 19,293 & & 20,988 & -27 & & & 19,854 & -3 & & & 9 & -74 & 9 & & 417 \\
\hline Pasco & 34,616 & 10 & & 15,812 & 2 & 12,528 & & 49,584 & -10 & & & & & 10,368 & -17 & 111 & -78 & 44 & 460 & 30 \\
\hline Pinellas & 7,622 & -37 & & & & & & 338 & 50 & 4 & & & & & & & & & 183 & \\
\hline Polk & 249,232 & 21 & 3,038 & 217,620 & 23 & 28,270 & & 35,554 & -31 & & & 18,783 & 18 & & & 44 & -82 & 35 & 650 & 5,476 \\
\hline St. Lucie & 112,346 & -31 & & 101,968 & -28 & & & 15,561 & 47 & 4 & & & & & & 15 & -74 & 14 & 119 & \\
\hline Sarasota & 13,343 & -24 & & 4,865 & 47 & 8,312 & & 4,458 & -39 & & & 3,637 & -17 & & & 9 & -87 & 2 & & \\
\hline Seminole & 17,949 & -4 & 29 & 1,233 & -18 & 16,680 & 7 & 1,262 & -39 & 13 & & 759 & -15 & 60 & & 38 & -7 & 12 & 269 & 84 \\
\hline Sumter & 14,022 & 44 & 2,378 & 127 & & 10,191 & 1,322 & 16,622 & -35 & & & 7,548 & -10 & 3,011 & -24 & 13 & -86 & 15 & 2,786 & \\
\hline Total & $2,159,559$ & 6 & 270,850 & $1,089,557$ & 5 & 723,148 & 3,153 & 473,631 & -9 & 13,476 & -43 & 172,856 & 4 & 151,768 & -19 & 707 & -71 & 299 & 8,525 & 30,906 \\
\hline
\end{tabular}


Table 6. Characteristics of principal operator, and primary occupation of farmers, central region of Florida, 2002, and percent change 1997-2002

\begin{tabular}{|c|c|c|c|c|c|c|c|c|c|c|}
\hline \multirow{2}{*}{ County } & \multirow{2}{*}{$\begin{array}{r}\text { Total operators } \\
\text { (Number) }\end{array}$} & \multirow{2}{*}{$\begin{array}{r}\text { Total women operators } \\
\text { (Number) }\end{array}$} & \multicolumn{2}{|c|}{ Farming primary occupation } & \multicolumn{2}{|c|}{ Other primary occupation } & \multicolumn{4}{|c|}{ Female farmers } \\
\hline & & & (Number) & (\% chg) & (Number) & (\% chg) & (Number) & (\% chg) & (Acres) & (\% chg) \\
\hline Brevard & 819 & 260 & 296 & 37 & 259 & -34 & 116 & 25 & 16,443 & 46 \\
\hline Citrus & 655 & 248 & 205 & 31 & 227 & -11 & 97 & 41 & 3,128 & -22 \\
\hline DeSoto & 1,567 & 415 & 606 & 47 & 547 & 3 & 150 & 1 & 40,829 & 34 \\
\hline Hardee & 1,591 & 398 & 602 & 12 & 540 & -28 & 163 & -11 & 27,891 & -10 \\
\hline Hernando & 950 & 341 & 335 & 31 & 282 & -28 & 132 & 10 & 7,848 & -7 \\
\hline Highlands & 1,479 & 416 & 590 & 46 & 445 & -28 & 156 & -1 & 44,575 & -41 \\
\hline Hillsborough & 4,412 & 1,449 & 1,608 & 3 & 1,361 & -36 & 589 & 1 & 25,110 & -6 \\
\hline Indian River & 685 & 186 & 264 & -13 & 216 & -2 & 84 & 24 & 7,326 & 88 \\
\hline Lake & 2,698 & 879 & 816 & 23 & 982 & -15 & 327 & 30 & 16,030 & -31 \\
\hline Manatee & 1,331 & 427 & 448 & 3 & 404 & -13 & 145 & -7 & 22,597 & 17 \\
\hline Okeechobee & 951 & 295 & 354 & 49 & 284 & -28 & 78 & -6 & 13,177 & -64 \\
\hline Orange & 1,346 & 463 & 459 & -17 & 442 & -14 & 188 & 6 & 9,059 & -26 \\
\hline Osceola & 793 & 249 & 275 & -6 & 244 & -29 & 102 & -2 & 29,333 & 2 \\
\hline Pasco & 1,816 & 617 & 620 & 14 & 602 & -24 & 235 & 0 & 12,021 & -16 \\
\hline Pinellas & 168 & 65 & 46 & -35 & 65 & -32 & 35 & -5 & 359 & 10 \\
\hline Polk & 4,216 & 995 & 1,623 & 39 & 1,491 & -25 & 436 & -6 & 66,576 & 11 \\
\hline St. Lucie & 742 & 207 & 265 & -12 & 212 & -31 & 68 & -12 & 12,629 & 107 \\
\hline Sarasota & 583 & 218 & 194 & 24 & 177 & -42 & 92 & 12 & 20,122 & -5 \\
\hline Seminole & 543 & 197 & 166 & -14 & 210 & -26 & 80 & -4 & 3,103 & 24 \\
\hline Sumter & 1,289 & 411 & 478 & 26 & 424 & -34 & 151 & -11 & 20,239 & -12 \\
\hline Total & 28,634 & 8,736 & 10,250 & 16 & 9,414 & -25 & 3,424 & 3 & 398,395 & -9 \\
\hline
\end{tabular}

Source: 2002 Census of Agriculture, Florida state and county data, Volume 1, Geographic area series, Part 9, AC-02-A-9, Issued June 2004, National Statistics Service, US Department of Agriculture 
Table 7. Type of business organization, central region of Florida, 2002, and percent change, 1997-2002

\begin{tabular}{|c|c|c|c|c|c|c|c|c|c|c|c|c|c|c|c|c|c|c|c|}
\hline \multirow[b]{2}{*}{ County } & \multicolumn{4}{|c|}{ Family or individual farms } & \multicolumn{4}{|c|}{ Partnership farms } & \multicolumn{4}{|c|}{ Family-held farm corporation } & \multicolumn{4}{|c|}{$\begin{array}{l}\text { Other than family held } \\
\text { farm corporation }\end{array}$} & \multicolumn{3}{|c|}{$\begin{array}{l}\text { Other cooperative, estate } \\
\text { or trust, institution, etc }\end{array}$} \\
\hline & 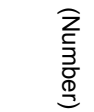 & $\begin{array}{l}\widehat{\circ} \\
\text { 今े } \\
\text { a् }\end{array}$ & 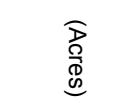 & $\begin{array}{l}\widehat{\circ} \\
\text { 今े } \\
\text { ấ }\end{array}$ & 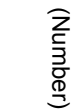 & $\begin{array}{l}\widehat{\circ} \\
\text { 今े } \\
\text { â }\end{array}$ & $\begin{array}{l}\text { 今 } \\
\text { 市 } \\
\text { क्र }\end{array}$ & $\begin{array}{l}\widehat{\circ} \\
\text { 今े } \\
\text { a }\end{array}$ & $\begin{array}{l}\widehat{z} \\
\underline{\mathbf{z}} \\
\overline{\mathbf{g}} \\
\underline{\underline{9}}\end{array}$ & $\begin{array}{l}\widehat{\circ} \\
\text { 今े } \\
\text { a }\end{array}$ & 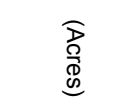 & $\begin{array}{l}\widehat{\circ} \\
\text { 今े } \\
\text { âd }\end{array}$ & $\begin{array}{l}\widehat{z} \\
\underline{\mathbf{z}} \\
\overline{\mathbf{g}} \\
\underline{\underline{9}}\end{array}$ & $\begin{array}{l}\widehat{\circ} \\
\stackrel{9}{c} \\
\text { a }\end{array}$ & 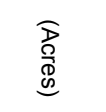 & $\begin{array}{l}\widehat{\circ} \\
\text { ⿳亠口冋े } \\
\text { a }\end{array}$ & 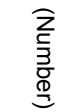 & $\begin{array}{l}\text { å } \\
\text { 今े } \\
\text { â }\end{array}$ & 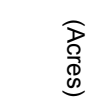 \\
\hline Brevard & 484 & 2 & 58,670 & -44 & 28 & -53 & 52,557 & 16 & 34 & -47 & 16,460 & -70 & 5 & -29 & & & 4 & 33 & \\
\hline Citrus & 401 & 8 & 38,257 & 0 & 7 & -67 & 4,365 & -19 & 18 & 38 & 3,268 & -26 & 1 & -50 & & & 5 & -17 & \\
\hline DeSoto & 954 & 34 & 215,204 & 43 & 94 & -28 & 75,581 & -7 & 77 & 0 & 76,775 & & 21 & 62 & 18,283 & -61 & 7 & 17 & 2,334 \\
\hline Hardee & 891 & -7 & 216,864 & 27 & 118 & -27 & 79,056 & 23 & 100 & -25 & 34,963 & -64 & 15 & -17 & 10,847 & 64 & 18 & -25 & 4,461 \\
\hline Hernando & 560 & 1 & & & 16 & -56 & 6,226 & 191 & 30 & -19 & 15,568 & 60 & 2 & -80 & & & 9 & 13 & \\
\hline Highlands & 807 & 8 & & & 66 & -25 & 26,858 & -81 & 134 & -9 & 305,971 & 58 & 20 & -23 & 3,472 & -85 & 8 & 0 & \\
\hline Hillsborough & 2,579 & -17 & 149,203 & 1 & 111 & -50 & 50,263 & 19 & 234 & -21 & 69,805 & 4 & 26 & -28 & 14,761 & 116 & 19 & -24 & 878 \\
\hline Indian River & 360 & 1 & & & 32 & -48 & 30,094 & -43 & 69 & -18 & 73,426 & -5 & 15 & -6 & 7,617 & 9 & 4 & -33 & \\
\hline Lake & 1,488 & 3 & 89,365 & -21 & 90 & -22 & 21,879 & 2 & 170 & -19 & 57,786 & 12 & 29 & -9 & 7,789 & 43 & 21 & 75 & 3,426 \\
\hline Manatee & 649 & -4 & 129,722 & 24 & 80 & 1 & 58,242 & 6 & 86 & -26 & 87,581 & 19 & 27 & 50 & 16,469 & -52 & 10 & 43 & 9,217 \\
\hline Okeechobee & 522 & 4 & 200,087 & 11 & 26 & -37 & 46,057 & 37 & 81 & -1 & 132,578 & -20 & 3 & -50 & & & 6 & 200 & \\
\hline Orange & 631 & -10 & & & 66 & -23 & 8,430 & -44 & 164 & -29 & 13,062 & -86 & 31 & 82 & & & 9 & -61 & 2,847 \\
\hline Osceola & 440 & -12 & 181,977 & 66 & 34 & -40 & 98,265 & 58 & 32 & -49 & 44,812 & -77 & 10 & -29 & & & 3 & -40 & \\
\hline Pasco & 1,085 & -4 & & & 44 & -45 & 7,204 & -72 & 86 & -17 & 42,277 & -25 & 3 & -81 & & & 4 & -56 & 25 \\
\hline Pinellas & 78 & -38 & 1,012 & -54 & 7 & -53 & 186 & -44 & 22 & -8 & 320 & & 1 & & & & 3 & 50 & \\
\hline Polk & 2,342 & 1 & 337,645 & 1 & 298 & -17 & 121,082 & 33 & 304 & -14 & 147,028 & 11 & 39 & -33 & 8,270 & -80 & 131 & 102 & 12,609 \\
\hline St. Lucie & 295 & -17 & & & 70 & -29 & 18,183 & -14 & 89 & -22 & 135,233 & -5 & 14 & -46 & 1,118 & & 9 & -44 & \\
\hline Sarasota & 274 & -26 & 49,447 & -20 & 33 & 14 & 26,430 & 44 & 43 & 2 & 43,568 & -6 & 11 & 0 & 1,198 & & 10 & -9 & 667 \\
\hline Seminole & 305 & -16 & & & 22 & 10 & 18,610 & 9 & 37 & -49 & 1,140 & -91 & 11 & -8 & 184 & 104 & 1 & -90 & \\
\hline Sumter & 833 & -12 & & & 31 & -3 & 20,078 & 31 & 31 & 7 & 49,819 & 42 & 2 & 0 & & & 5 & -58 & \\
\hline Total & 15,978 & -4 & $1,667,453$ & -19 & 1,273 & -29 & 769,646 & -5 & 1,841 & -20 & $1,351,440$ & -10 & 286 & -16 & 90,008 & -49 & 286 & 10 & 36,464 \\
\hline
\end{tabular}

Source: 2002 Census of Agriculture, Florida state and county data, Volume 1, Geographic area series, Part 9, AC-02-A-9, Issued June 2004, National Statistics Service, US Department of Agriculture 
Table 8. Ethnicity of farm operators, central region of Florida, 2002

\begin{tabular}{|c|c|c|c|c|c|c|c|c|c|c|c|c|}
\hline \multirow{2}{*}{ County } & \multicolumn{3}{|c|}{ White } & \multicolumn{3}{|c|}{ Black or African American } & \multicolumn{3}{|c|}{ Spanish, Hispanic, or Latino origin } & \multicolumn{3}{|c|}{ Other ethnic groups * } \\
\hline & (Farms) & (Operators) & (Acres) & (Farms) & (Operators) & (Acres) & (Farms) & (Operators) & (Acres) & (Farms) & (Operators) & (Acres) \\
\hline Brevard & 549 & 775 & 187465 & 9 & 9 & 174 & 9 & 12 & 302 & 11 & 11 & 588 \\
\hline Citrus & 420 & 633 & 46563 & & & & 10 & 10 & 196 & 16 & 16 & 698 \\
\hline De Soto & 1106 & 1488 & 387111 & 7 & 7 & 125 & 24 & 25 & 11567 & 52 & 54 & 3740 \\
\hline Hardee & 1138 & 1570 & 346054 & & & & 55 & 68 & 1213 & 5 & 5 & 30 \\
\hline Hernando & 611 & 902 & 63977 & 5 & 5 & 1141 & 45 & 50 & 2645 & 21 & 21 & 239 \\
\hline Highlands & 1007 & 1387 & 576514 & 27 & 29 & 4251 & 52 & 60 & 11582 & 13 & 15 & 155 \\
\hline Hillsborough & 2868 & 4132 & 276374 & 52 & 60 & 1744 & 230 & 290 & 11996 & 89 & 113 & 3109 \\
\hline Indian River & 468 & 650 & 190716 & 14 & 14 & 645 & 17 & 17 & & 3 & 3 & 30 \\
\hline Lake & 1732 & 2518 & 177518 & 33 & 39 & 1100 & 110 & 141 & 9123 & 59 & 69 & 2475 \\
\hline Manatee & 825 & 1216 & 298053 & 5 & 5 & 40 & 41 & 52 & 16537 & 29 & 46 & 3998 \\
\hline Okeechobee & 638 & 926 & 392495 & & & & 39 & 46 & 35469 & 8 & 8 & 3042 \\
\hline Orange & 843 & 1211 & 144028 & 7 & 7 & & 38 & 38 & 1016 & 65 & 83 & 2929 \\
\hline Osceola & 509 & 740 & 651755 & 1 & 1 & & 15 & 18 & 297 & 18 & 20 & 873 \\
\hline Pasco & 1203 & 1734 & 168050 & 12 & 12 & 337 & 97 & 119 & 3544 & 19 & 22 & 1654 \\
\hline Pinellas & 107 & 156 & & & & & 9 & 9 & 23 & 5 & 5 & 4 \\
\hline Polk & 3082 & 4106 & 623354 & 16 & 22 & 9569 & 105 & 119 & 5897 & 24 & 27 & 437 \\
\hline St. Lucie & 468 & 694 & 221205 & 3 & 3 & 15 & 29 & 33 & 9181 & 14 & 24 & 339 \\
\hline Sarasota & 362 & 534 & 121024 & 3 & 3 & 33 & 22 & 22 & 4654 & 15 & 15 & 406 \\
\hline Seminole & 358 & 507 & 27839 & 12 & 16 & 62 & 2 & 3 & & 9 & 11 & 98 \\
\hline Sumter & 846 & 1194 & 185790 & 32 & 36 & 1386 & 44 & 53 & 2762 & 28 & 28 & 789 \\
\hline Total & 19,140 & 27,073 & $5,085,885$ & 238 & 268 & 20,622 & 993 & 1,185 & 128,004 & 503 & 596 & 25,633 \\
\hline
\end{tabular}

* (American Indian, Alaska Native, Native Hawaiian, Other Pacific Islander \& Asian)

Source: 2002 Census of Agriculture, Florida state and county data, Volume 1, Geographic area series, Part 9, AC-02-A-9, Issued June 2004, National Statistics Service, US Department of Agriculture 
Table 9. Output impacts of agriculture and natural resource industries, central region of Florida, 2002 data

\begin{tabular}{|c|c|c|c|c|c|c|c|c|c|c|c|c|c|c|c|c|c|c|c|c|c|}
\hline Industry Group & 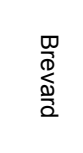 & 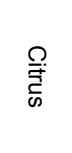 & 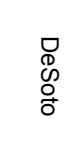 & $\begin{array}{l}\frac{I}{D} \\
\frac{D}{0} \\
\stackrel{D}{D} \\
\mathbb{D}\end{array}$ & 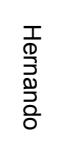 & 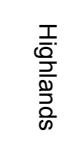 & 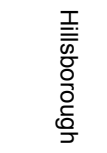 & 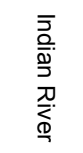 & $\frac{\bar{D}}{\hat{\alpha}}$ & 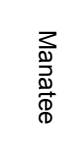 & 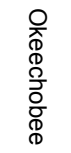 & 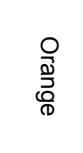 & $\begin{array}{l}\text { O } \\
\varnothing \\
0 \\
0 \\
\frac{0}{Q}\end{array}$ & 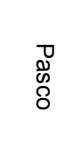 & 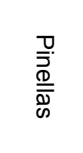 & $\begin{array}{l}\frac{0}{2} \\
\frac{O}{\pi}\end{array}$ & 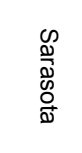 & 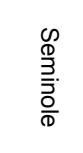 & 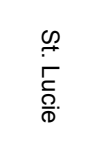 & $\begin{array}{l}\infty \\
\coprod_{3} \\
\stackrel{\vec{\Phi}}{=}\end{array}$ & $\begin{array}{c}\text { Grand } \\
\text { Total }\end{array}$ \\
\hline Agricultural Inputs \& Services & & & & \multicolumn{18}{|c|}{ (\$Million) } \\
\hline Agriculture \& forestry activities & 24.4 & 4.1 & 121.4 & 77.6 & 1.1 & 160.2 & 213.4 & 171.3 & 50.6 & 24.5 & 2.7 & 117.1 & 6.7 & 42.3 & 9.7 & 369.3 & 13.6 & 8.3 & 241.9 & 0.3 & $1,660.3$ \\
\hline Farm machinery \& equipment mfg & 0.0 & 0.0 & 0.0 & 0.0 & 0.0 & 0.0 & 13.7 & 0.0 & 0.4 & 0.0 & 0.0 & 0.7 & 3.4 & 0.0 & 2.6 & 23.3 & 0.0 & 0.0 & 3.5 & 0.0 & 47.7 \\
\hline Fertilizer, mixing only, mfg & 0.0 & 0.0 & 0.0 & 0.0 & 0.0 & 0.0 & 24.9 & 0.0 & 31.7 & 6.2 & 8.0 & 92.1 & 0.0 & 11.8 & 0.0 & 49.5 & 0.0 & 6.2 & 9.8 & 0.6 & 240.8 \\
\hline Food product machinery mfg & 1.3 & 0.0 & 0.0 & 0.0 & 0.0 & 0.0 & 4.9 & 0.0 & 0.0 & 4.6 & 0.0 & 5.5 & 0.0 & 7.9 & 1.3 & 27.1 & 0.0 & 0.0 & 0.0 & 0.0 & 52.8 \\
\hline Lawn/garden equipment mfg & 0.0 & 0.0 & 0.0 & 0.0 & 0.0 & 0.0 & 0.0 & 0.0 & 0.0 & 1.0 & 0.0 & 0.0 & 0.0 & 0.0 & 0.0 & 0.0 & 0.0 & 0.0 & 0.0 & 0.0 & 1.0 \\
\hline New farm housing units/additions & 20.1 & 4.7 & 0.6 & 0.4 & 4.5 & 2.1 & 48.4 & 5.0 & 12.7 & 11.7 & 0.8 & 46.8 & 6.0 & 10.5 & 36.0 & 18.5 & 20.0 & 22.5 & 6.5 & 1.1 & 278.6 \\
\hline Nitrogenous fertilizer mfg & 0.0 & 0.0 & 0.0 & 0.0 & 0.0 & 58.8 & 159.5 & 66.5 & 0.0 & 0.0 & 0.0 & 0.0 & 0.0 & 8.7 & 0.0 & 10.0 & 0.0 & 0.0 & 0.0 & 0.0 & 303.5 \\
\hline Pesticide \& other ag chemical mfg & 0.0 & 31.8 & 0.0 & 0.0 & 0.0 & 0.0 & 6.0 & 0.0 & 0.0 & 23.1 & 0.0 & 15.6 & 0.0 & 0.0 & 26.5 & 25.4 & 0.0 & 0.0 & 0.0 & 0.0 & 128.3 \\
\hline Phosphatic fertilizer mfg & 0.0 & 0.0 & 0.0 & 0.0 & 0.0 & 0.0 & 947.6 & 0.0 & 0.0 & 0.0 & 0.0 & 0.0 & 0.0 & 0.0 & 0.0 & $2,173.6$ & 0.0 & 0.0 & 0.0 & 0.0 & $3,121.2$ \\
\hline Veterinary services & 81.8 & 8.9 & 2.3 & 0.4 & 13.9 & 3.7 & 75.9 & 12.0 & 17.0 & 35.8 & 0.6 & 53.4 & 4.3 & 26.5 & 81.0 & 33.3 & 34.1 & 56.0 & 9.4 & 2.8 & 552.8 \\
\hline Total & 127.6 & 49.5 & 124.3 & 78.4 & 19.6 & 224.7 & $1,494.2$ & 254.7 & 112.3 & 106.9 & 12.1 & 331.2 & 20.4 & 107.6 & 157.2 & $2,729.9$ & 67.6 & 93.0 & 271.1 & 4.7 & $6,387.0$ \\
\hline \multicolumn{22}{|l|}{ Environmental Horticulture } \\
\hline Greenhouse/nursery production & 13.4 & 2.1 & 13.3 & 26.7 & 2.9 & 48.8 & 252.3 & 10.0 & 175.5 & 61.3 & 7.9 & 277.6 & 23.6 & 12.5 & 25.8 & 38.6 & 13.9 & 32.1 & 3.8 & 14.5 & $1,056.8$ \\
\hline Landscape services ( $70 \%$ bldgs) & 81.5 & 20.6 & 1.5 & 1.6 & 29.0 & 9.5 & 345.5 & 41.7 & 45.6 & 77.0 & 3.8 & 524.7 & 36.6 & 60.1 & 280.3 & 67.7 & 134.0 & 148.4 & 50.5 & 1.4 & $1,961.0$ \\
\hline Total & 94.9 & 22.6 & 14.8 & 28.3 & 31.9 & 58.3 & 597.8 & 51.7 & 221.1 & 138.3 & 11.8 & 802.2 & 60.2 & 72.6 & 306.1 & 106.3 & 148.0 & 180.5 & 54.3 & 15.9 & $3,017.9$ \\
\hline \multicolumn{22}{|l|}{ Fishing \& Seafood Products } \\
\hline Fishing & 0.9 & 5.7 & 0.0 & 0.0 & 5.8 & 0.0 & 39.8 & 14.4 & 2.4 & 0.0 & 0.0 & 0.0 & 16.0 & 1.6 & 22.6 & 0.0 & 6.2 & 1.1 & 3.4 & 0.0 & 120.1 \\
\hline Seafood product prep/packaging & 7.6 & 2.0 & 0.0 & 0.0 & 0.0 & 0.0 & 391.0 & 0.0 & 0.0 & 0.0 & 0.0 & 0.0 & 0.0 & 1.0 & 5.8 & 152.5 & 0.0 & 0.0 & 0.0 & 0.0 & 559.8 \\
\hline Total & 8.6 & 7.7 & 0.0 & 0.0 & 5.8 & 0.0 & 430.8 & 14.4 & 2.4 & 0.0 & 0.0 & 0.0 & 16.0 & 2.6 & 28.4 & 152.5 & 6.2 & 1.1 & 3.4 & 0.0 & 679.9 \\
\hline \multicolumn{22}{|l|}{ Forestry, Wood \& Paper Product Mfg } \\
\hline All other converted paper product mfg & 0.1 & 0.0 & 0.0 & 0.0 & 0.0 & 0.0 & 14.2 & 0.0 & 0.0 & 0.0 & 0.0 & 4.8 & 0.0 & 0.0 & 1.3 & 3.9 & 0.0 & 0.0 & 0.0 & 0.0 & 24.3 \\
\hline Coated \& laminated paper/packaging & 0.0 & 0.0 & 0.0 & 0.0 & 0.0 & 0.0 & 16.3 & 0.0 & 0.0 & 2.7 & 0.0 & 7.0 & 0.0 & 0.0 & 29.7 & 11.1 & 1.8 & 4.4 & 0.0 & 0.0 & 72.8 \\
\hline Coated \& uncoated paper bag mfg & 0.1 & 0.0 & 0.0 & 0.0 & 0.0 & 0.0 & 12.2 & 0.0 & 0.0 & 0.0 & 0.0 & 1.0 & 0.0 & 0.0 & 0.0 & 0.0 & 0.0 & 0.0 & 0.0 & 0.0 & 13.3 \\
\hline Die-cut paper office supplies mfg & 0.0 & 0.0 & 0.0 & 0.0 & 0.0 & 0.0 & 23.5 & 0.0 & 0.0 & 0.0 & 0.0 & 3.0 & 0.0 & 0.0 & 0.0 & 0.0 & 0.0 & 0.0 & 0.0 & 0.0 & 26.5 \\
\hline Engineered wood \& truss mfg & 85.0 & 8.4 & 0.0 & 0.0 & 24.8 & 0.0 & 49.1 & 0.7 & 12.0 & 0.0 & 0.0 & 42.6 & 0.3 & 4.3 & 10.6 & 80.9 & 14.7 & 28.8 & 34.1 & 1.2 & 397.3 \\
\hline Envelope manufacturing & 0.0 & 0.0 & 0.0 & 0.0 & 0.0 & 0.0 & -3.9 & 0.0 & 0.0 & 0.0 & 0.0 & -0.4 & 0.0 & 0.0 & 0.1 & 0.0 & 0.0 & 0.0 & 0.0 & 0.0 & -4.2 \\
\hline Forest nurseries/products/timber & 4.3 & 9.4 & 0.0 & 0.0 & 0.0 & 24.9 & 9.8 & 34.4 & 9.1 & 0.0 & 0.0 & 14.2 & 0.0 & 4.4 & 0.0 & 0.0 & 0.0 & 0.0 & 0.0 & 0.0 & 110.4 \\
\hline Logging & 0.0 & 0.0 & 3.5 & 0.5 & 2.4 & 0.0 & 0.0 & 5.9 & 15.8 & 0.0 & 0.0 & 0.0 & 9.8 & 4.6 & 0.0 & 4.7 & 0.0 & 0.8 & 0.0 & 11.4 & 59.3 \\
\hline Miscellaneous wood product mfg & 0.6 & 0.0 & 0.0 & 1.2 & 0.0 & 0.0 & 2.7 & 0.0 & 0.0 & 0.0 & 0.0 & 3.3 & 0.4 & 0.0 & 5.5 & 18.3 & 0.0 & 1.3 & 0.0 & 0.0 & 33.3 \\
\hline Other millwork, including flooring & 0.5 & 0.0 & 0.0 & 0.0 & 0.5 & 0.0 & 17.1 & 4.6 & 0.2 & 16.8 & 0.0 & 1.9 & 0.0 & 0.5 & 2.3 & 20.3 & 3.7 & 0.7 & 0.4 & 0.0 & 69.4 \\
\hline Paper \& paperboard mills & 1.9 & 0.0 & 0.0 & 0.0 & 0.0 & 0.0 & 6.5 & 0.0 & 0.0 & 0.0 & 0.0 & 0.0 & 0.0 & 0.0 & 0.0 & 0.0 & 0.0 & 0.0 & 0.0 & 0.8 & 9.3 \\
\hline Paperboard container mfg & 0.2 & 0.0 & 0.0 & 0.0 & 0.0 & 49.9 & 291.4 & 0.0 & 0.0 & 25.6 & 0.0 & 102.6 & 0.0 & 6.5 & 161.9 & 182.0 & 2.3 & 2.2 & 0.0 & 0.0 & 824.4 \\
\hline Prefabricated wood building $\mathrm{mfg}$ & 0.0 & 0.0 & 2.7 & 0.0 & 0.0 & 0.0 & 1.0 & 0.0 & 0.0 & 0.0 & 0.0 & 0.0 & 0.0 & 0.0 & 0.0 & 3.9 & 0.0 & 1.4 & 0.0 & 0.0 & 8.9 \\
\hline Reconstituted wood product mfg & 0.0 & 0.0 & 0.0 & 0.0 & 0.0 & 0.0 & 1.4 & 0.0 & 0.0 & 0.0 & 0.0 & 0.0 & 0.0 & 0.0 & 0.9 & 0.0 & 0.0 & 0.0 & 0.0 & 0.0 & 2.3 \\
\hline Sanitary paper product mfg & 0.0 & 0.0 & 0.0 & 0.0 & 0.0 & 0.0 & 0.0 & 0.0 & 0.0 & 0.0 & 0.0 & 185.4 & 0.0 & 0.0 & 0.0 & 0.0 & 0.0 & 0.0 & 0.0 & 0.0 & 185.4 \\
\hline Sawmills & 0.0 & 0.0 & 0.0 & 2.4 & 0.0 & 0.0 & 1.8 & 0.0 & 0.7 & 0.0 & 0.0 & 6.2 & 8.7 & 7.8 & 3.7 & 0.0 & 0.0 & 0.0 & 0.0 & 19.8 & 50.9 \\
\hline Stationery \& related product mfg & 0.0 & 0.0 & 0.0 & 0.0 & 0.0 & 0.0 & 14.8 & 0.0 & 0.0 & 0.0 & 0.0 & 0.0 & 0.0 & 0.0 & 0.0 & 0.0 & 0.0 & 0.0 & 0.0 & 0.0 & 14.8 \\
\hline Surface-coated paperboard mfg & 0.0 & 0.0 & 0.0 & 0.0 & 0.0 & 0.0 & 0.0 & 0.0 & 0.0 & 0.0 & 0.0 & 4.1 & 0.0 & 0.0 & 0.0 & 0.0 & 0.0 & 0.0 & 0.0 & 0.0 & 4.1 \\
\hline Veneer \& plywood mfg & 0.0 & 0.0 & 0.0 & 0.0 & 0.0 & 0.0 & 0.0 & 0.0 & 0.0 & 0.0 & 0.0 & 0.0 & 0.0 & 0.0 & 0.0 & 2.5 & 0.0 & 0.0 & 0.0 & 0.0 & 2.5 \\
\hline Wood container/pallet mfg & 0.6 & 0.0 & 0.0 & 0.0 & 0.0 & 8.0 & 4.2 & 0.0 & 18.4 & 0.2 & 0.0 & 6.5 & 0.0 & 1.3 & 4.9 & 27.5 & 0.1 & 1.1 & 0.3 & 0.0 & 73.2 \\
\hline Wood preservation & 0.0 & 0.0 & 0.0 & 4.6 & 0.0 & 0.0 & 76.9 & 0.0 & 0.0 & 0.0 & 0.0 & 7.9 & 0.0 & 0.8 & 2.5 & 39.4 & 0.0 & 0.0 & 0.0 & 0.0 & 132.1 \\
\hline Wood windows/doors mfg & 0.8 & 0.0 & 0.0 & 0.0 & 0.0 & 0.0 & 63.8 & 0.0 & 36.2 & 0.0 & 0.0 & 11.9 & 28.5 & 5.7 & 15.9 & 0.0 & 82.5 & 49.8 & 0.6 & 0.0 & 295.7 \\
\hline Total & 94.1 & 17.8 & 6.2 & 8.7 & 27.7 & 82.8 & 602.9 & 45.5 & 92.3 & 45.3 & 0.0 & 401.8 & 47.7 & 35.9 & 239.1 & 394.3 & 105.0 & 90.4 & 35.4 & 33.3 & $2,406.1$ \\
\hline
\end{tabular}


Table 9 (continued). Output impacts of agriculture and natural resource industries, central region of Florida, 2002 data

\begin{tabular}{|c|c|c|c|c|c|c|c|c|c|c|c|c|c|c|c|c|c|c|c|c|c|}
\hline Industry Group & 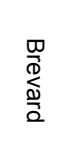 & $\frac{\rho}{\stackrel{\rho}{F}}$ & $\begin{array}{l}\text { O } \\
\mathbb{\infty} \\
\stackrel{0}{0} \\
\stackrel{0}{0}\end{array}$ & $\begin{array}{l}\frac{I}{2} \\
\frac{N}{2} \\
\frac{D}{D} \\
\mathbb{D}\end{array}$ & $\begin{array}{l}\frac{1}{D} \\
\mathbb{D} \\
3 \\
\frac{0}{3} \\
0 \\
0\end{array}$ & 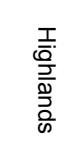 & $\begin{array}{l}\text { I } \\
\underline{\overline{\bar{D}}} \\
\underline{0} \\
\underline{0} \\
\underline{0} \\
\underline{\underline{0}}\end{array}$ & 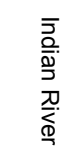 & $\frac{\bar{D}}{\hat{\phi}}$ & 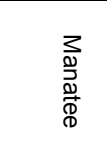 & 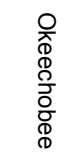 & $\begin{array}{l}\mathrm{O} \\
\stackrel{\mathbb{D}}{\vec{D}} \\
\stackrel{\overrightarrow{0}}{\mathbb{D}}\end{array}$ & $\begin{array}{l}\text { O } \\
\text { Qn } \\
\mathbb{0} \\
\frac{O}{\mathscr{N}}\end{array}$ & 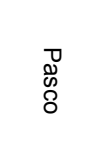 & 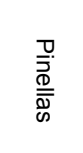 & $\frac{0}{\frac{0}{\pi}}$ & $\begin{array}{l}\mathscr{W} \\
\stackrel{D}{D} \\
\mathbb{D} \\
\mathscr{O} \\
\stackrel{D}{D}\end{array}$ & $\begin{array}{l}\text { W } \\
\mathbb{D} \\
3 \\
\frac{3}{\mathbb{D}}\end{array}$ & 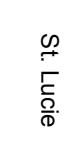 & 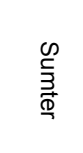 & $\begin{array}{l}\text { Grand } \\
\text { Total }\end{array}$ \\
\hline Fruit \& Vegetable Farming \& Processing & & & & \multicolumn{18}{|c|}{ (\$Million) } \\
\hline Frozen food manufacturing & 0.0 & 0.0 & 65.3 & 0.0 & 1.5 & 41.4 & 76.1 & 46.0 & 19.1 & 0.0 & 0.0 & 249.5 & 0.0 & 170.8 & 0.0 & 968.2 & 0.0 & 0.0 & 18.3 & 0.0 & $1,656.4$ \\
\hline Fruit \& vegetable canning/drying & 0.0 & 0.0 & 0.0 & 37.0 & 0.0 & 10.0 & 27.6 & 0.0 & 227.0 & $1,731.0$ & 0.0 & 14.9 & 5.8 & 0.0 & 245.4 & 248.1 & 2.4 & 0.0 & 211.4 & 0.0 & $2,760.6$ \\
\hline Fruit farming & 20.7 & 4.1 & 206.0 & 134.7 & 2.6 & 218.6 & 626.0 & 152.4 & 59.1 & 94.4 & 60.2 & 33.9 & 34.5 & 44.9 & 1.5 & 510.9 & 17.4 & 5.2 & 111.2 & 1.3 & $2,339.7$ \\
\hline Vegetable \& melon farming & 3.6 & 0.5 & 9.5 & 31.5 & 7.9 & 7.0 & 304.5 & 5.2 & 1.3 & 419.6 & 50.7 & 1.8 & 11.7 & 8.7 & 1.6 & 8.3 & 10.1 & 0.1 & 1.1 & 6.6 & 891.3 \\
\hline Total & 24.3 & 4.6 & 280.9 & 203.3 & 12.1 & 277.0 & $1,034.1$ & 203.5 & 306.5 & $2,245.0$ & 110.9 & 300.1 & 52.1 & 224.4 & 248.5 & $1,735.5$ & 29.9 & 5.4 & 342.0 & 7.9 & $7,648.0$ \\
\hline \multicolumn{22}{|l|}{ Grain \& Oilseed Farming \& Processing } \\
\hline Flour milling & 0.0 & 0.0 & 0.0 & 0.0 & 0.0 & 0.0 & 59.4 & 0.0 & 0.0 & 0.0 & 0.0 & 0.0 & 0.0 & 0.0 & 0.0 & 3.1 & 0.0 & 0.0 & 0.0 & 0.0 & 62.5 \\
\hline Grain farming & 0.0 & 0.0 & 0.0 & 0.0 & 0.0 & 0.0 & 0.0 & 0.0 & 0.0 & 0.0 & 0.0 & 0.0 & 0.0 & 0.0 & 0.0 & 0.0 & 0.0 & 0.0 & 0.0 & 0.0 & 0.1 \\
\hline Total & 0.0 & 0.0 & 0.0 & 0.0 & 0.0 & 0.0 & 59.5 & 0.0 & 0.0 & 0.0 & 0.0 & 0.0 & 0.0 & 0.0 & 0.0 & 3.1 & 0.0 & 0.0 & 0.0 & 0.0 & 62.6 \\
\hline \multicolumn{22}{|c|}{ Livestock \& Dairy Farming \& Animal Products Mfg } \\
\hline Animal production, except cattle \& poultry & 2.2 & 3.3 & 3.3 & 1.2 & 1.5 & 1.2 & 43.9 & 0.9 & 16.3 & 8.2 & 4.2 & 4.2 & 3.7 & 2.6 & 0.5 & 13.6 & 2.7 & 0.9 & 0.6 & 4.1 & 119.0 \\
\hline Animal, except poultry, slaughtering & 0.0 & 0.0 & 2.8 & 0.0 & 0.0 & 0.0 & 32.8 & 0.0 & 0.0 & 0.0 & 0.0 & 4.8 & 0.0 & 7.0 & 21.6 & 0.0 & 2.4 & 5.7 & 0.0 & 131.2 & 208.2 \\
\hline Cattle ranching \& farming & 9.6 & 13.8 & 13.9 & 35.0 & 16.5 & 25.1 & 47.0 & 4.1 & 12.3 & 41.2 & 106.0 & 3.4 & 29.9 & 43.3 & 0.0 & 22.4 & 10.4 & 3.5 & 1.7 & 23.5 & 462.6 \\
\hline Cheese manufacturing & 0.0 & 0.0 & 0.0 & 0.0 & 0.0 & 0.0 & 18.9 & 0.0 & 0.0 & 0.0 & 0.0 & 13.2 & 0.0 & 0.0 & 0.0 & 0.0 & 0.0 & 2.3 & 0.0 & 0.0 & 34.5 \\
\hline Creamery butter manufacturing & 1.0 & 0.0 & 0.0 & 0.0 & 0.0 & 0.0 & 0.0 & 0.0 & 0.0 & 0.0 & 0.0 & 0.0 & 0.0 & 0.0 & 0.0 & 0.0 & 0.0 & 0.0 & 0.0 & 0.0 & 1.0 \\
\hline $\begin{array}{l}\text { Dry/condensed/evaporated dairy } \\
\text { products }\end{array}$ & 0.0 & 0.0 & 0.0 & 0.0 & 0.0 & 0.0 & 0.0 & 0.0 & 0.0 & 0.0 & 0.0 & 0.0 & 0.0 & 0.0 & 16.2 & 0.0 & 0.0 & 0.0 & 0.0 & 0.0 & 16.2 \\
\hline Fluid milk manufacturing & 1.6 & 0.0 & 0.0 & 0.0 & 0.0 & 0.0 & 62.6 & 0.0 & 0.0 & 0.0 & 0.0 & 253.7 & 0.0 & 0.0 & 0.0 & 60.8 & 0.0 & 0.0 & 0.0 & 0.0 & 378.7 \\
\hline Ice cream \& frozen dessert mfg & 0.0 & 0.0 & 0.0 & 0.0 & 0.0 & 0.0 & 2.6 & 0.0 & 0.0 & 0.0 & 0.0 & 0.0 & 0.0 & 0.0 & 248.3 & 0.0 & 1.7 & 0.0 & 0.0 & 0.0 & 252.5 \\
\hline Meat processed from carcasses & 0.0 & 0.0 & 0.0 & 0.0 & 0.0 & 0.0 & 170.6 & 0.0 & 0.0 & 0.0 & 0.0 & 0.0 & 0.0 & 0.0 & 5.5 & 23.8 & 0.6 & 0.0 & 0.0 & 0.0 & 200.5 \\
\hline Poultry \& egg production & 0.0 & 0.0 & 0.0 & 0.0 & 6.7 & 0.0 & 39.7 & 0.0 & 13.8 & 0.0 & 0.0 & 0.0 & 0.7 & 15.2 & 0.0 & 17.7 & 4.5 & 0.0 & 0.0 & 9.4 & 107.6 \\
\hline Poultry processing & 0.0 & 0.0 & 0.0 & 0.0 & 0.0 & 0.0 & 0.7 & 0.0 & 0.0 & 0.0 & 0.0 & 0.0 & 0.0 & 0.0 & 0.0 & 0.0 & 0.0 & 0.0 & 0.0 & 0.0 & 0.7 \\
\hline Rendering \& meat byproduct processing & 0.0 & 0.0 & 0.0 & 0.0 & 0.0 & 0.0 & 31.2 & 0.0 & 0.0 & 0.0 & 0.0 & 1.9 & 0.0 & 0.0 & 0.0 & 0.0 & 0.0 & 0.0 & 0.0 & 0.0 & 33.1 \\
\hline Total & 14.4 & 17.1 & 20.0 & 36.2 & 24.7 & 26.4 & 449.9 & 5.0 & 42.4 & 49.4 & 110.2 & 281.1 & 34.3 & 68.2 & 292.0 & 138.3 & 22.2 & 12.3 & 2.3 & 168.1 & $1,814.4$ \\
\hline \multicolumn{22}{|l|}{ Mining } \\
\hline Drilling oil \& gas wells & 0.3 & & 0.0 & 0.0 & 0.0 & 0.5 & 2.1 & 0.0 & 0.3 & 0.6 & 0.3 & 1.1 & 0.0 & 0.0 & 0.0 & 3.0 & 5.9 & 0.8 & 0.0 & 0.0 & 15.1 \\
\hline Oil \& gas extraction & 0.0 & 0.0 & 0.0 & 0.0 & 0.0 & 0.0 & 2.3 & 0.0 & 0.0 & 0.0 & 0.0 & 0.4 & 0.0 & 0.2 & 96.6 & 0.0 & 0.0 & 5.3 & 1.8 & 0.0 & 106.6 \\
\hline Other nonmetallic mineral mining & 0.3 & 0.3 & 0.0 & 35.9 & 0.0 & 13.2 & 21.8 & 2.2 & 57.8 & 0.1 & 0.0 & 2.2 & 0.0 & 0.2 & 0.0 & 530.5 & 0.0 & 0.0 & 0.0 & 0.0 & 664.6 \\
\hline Sand/gravel/clay/refractory mining & 0.0 & 1.0 & 0.0 & 0.0 & 1.1 & 0.0 & 3.4 & 5.5 & 19.3 & 0.5 & 0.2 & 5.3 & 0.0 & 0.0 & 10.0 & 52.1 & 0.0 & 0.0 & 2.6 & 0.0 & 100.9 \\
\hline Stone mining \& quarrying & 9.5 & 7.0 & 0.0 & 0.0 & 68.0 & 0.0 & 6.2 & 0.0 & 0.6 & 0.0 & 0.0 & 0.0 & 0.0 & 6.4 & 0.0 & 35.0 & 5.5 & 0.0 & 3.3 & 16.9 & 158.4 \\
\hline Support activities for oil \& gas operations & 0.5 & 0.0 & 0.0 & 0.0 & 0.0 & 0.0 & 0.3 & 0.0 & 0.1 & 0.0 & 0.0 & 0.1 & 0.0 & 0.0 & 0.0 & 0.0 & 0.0 & 0.1 & 0.0 & 0.0 & 1.2 \\
\hline Support activities for other mining & 0.0 & 0.0 & 0.0 & 0.0 & 0.0 & 0.0 & 0.0 & 0.0 & 0.0 & 0.0 & 0.0 & 0.0 & 0.0 & 0.0 & 1.8 & 0.0 & 0.0 & 0.0 & 0.0 & 0.0 & 1.8 \\
\hline Total & 10.6 & 8.3 & 0.0 & 35.9 & 69.1 & 13.6 & 36.2 & 7.7 & 78.1 & 1.1 & 0.6 & 9.1 & 0.0 & 6.9 & 108.4 & 620.7 & 11.4 & 6.3 & 7.7 & 16.9 & $1,048.6$ \\
\hline
\end{tabular}


Table 9 (continued). Output impacts of agriculture and natural resource industries, central region of Florida, 2002 data

\begin{tabular}{|c|c|c|c|c|c|c|c|c|c|c|c|c|c|c|c|c|c|c|c|}
\hline Industry Group & $\begin{array}{l}\frac{w}{\mathbb{D}} \\
\stackrel{\$}{2} \\
\frac{2}{2}\end{array}$ & $\begin{array}{l}\stackrel{\rho}{\nexists} \\
\text { 节 }\end{array}$ & $\begin{array}{l}\text { O } \\
\mathbb{\infty} \\
0 \\
O \\
0 \\
0\end{array}$ & $\begin{array}{l}\frac{I}{2} \\
\frac{N}{20} \\
\frac{D}{D} \\
\mathbb{D}\end{array}$ & 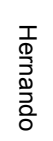 & 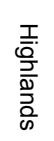 & $\begin{array}{l}\text { I } \\
\overline{\bar{D}} \\
\text { ㅁ } \\
\text { 어 } \\
\text { 음 }\end{array}$ & 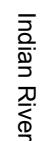 & $\frac{\bar{D}}{\overline{0}}$ & 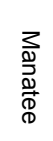 & 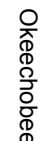 & 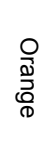 & 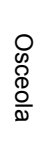 & 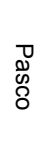 & $\stackrel{\overline{0}}{\omega}$ & $\begin{array}{l}\text { D } \\
\frac{O}{\pi}\end{array}$ & 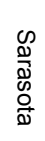 & & $\begin{array}{l}\text { Grand } \\
\text { Total }\end{array}$ \\
\hline
\end{tabular}

\begin{tabular}{|c|c|c|c|c|c|c|c|c|c|c|c|c|c|c|c|c|c|c|c|c|c|}
\hline \multirow[b]{2}{*}{ Other Crop Farming } & \multicolumn{21}{|c|}{ (\$Million) } \\
\hline & 0.2 & 0.5 & 1.3 & 1.8 & 1.8 & 1.5 & 3.1 & 0.0 & 1.5 & 1.6 & 2.7 & 0.3 & 0.7 & 3.2 & 0.0 & 1.7 & 0.1 & 0.2 & 0.0 & 4.1 & 26.2 \\
\hline \multicolumn{22}{|l|}{ Other Food Product Manufacturing } \\
\hline All other food manufacturing & 0.0 & 0.0 & 0.0 & 0.0 & 0.0 & 0.0 & 1.1 & 19.9 & 0.0 & 0.0 & 0.0 & 1.5 & 0.4 & 0.0 & 9.3 & 0.0 & 0.8 & 1.6 & 0.2 & 0.0 & 34.9 \\
\hline Bread/bakery, except frozen, mfg & 2.1 & 0.0 & 0.0 & 0.0 & 0.8 & 1.9 & 70.1 & 1.9 & 2.6 & 54.8 & 1.7 & 150.8 & 8.3 & 3.0 & 14.7 & 267.0 & 10.8 & 2.4 & 0.7 & 0.0 & 593.6 \\
\hline Breweries & 0.0 & 0.0 & 0.0 & 0.0 & 0.0 & 0.0 & 84.1 & 0.0 & 0.6 & 0.0 & 0.0 & 0.0 & 0.0 & 0.0 & 0.0 & 78.8 & 0.0 & 0.0 & 0.0 & 0.0 & 163.5 \\
\hline Coffee \& tea manufacturing & 0.0 & 0.0 & 0.0 & 0.0 & 0.0 & 0.0 & 30.0 & 0.0 & 0.0 & 0.0 & 0.0 & 2.3 & 0.0 & 0.0 & 3.5 & 0.0 & 0.0 & 0.0 & 0.0 & 0.0 & 35.8 \\
\hline Cookie $\&$ cracker manufacturing & 0.0 & 0.0 & 0.0 & 0.0 & 0.0 & 0.0 & 239.0 & 0.0 & 0.0 & 1.5 & 0.0 & 1.3 & 0.0 & 0.0 & 0.0 & 2.5 & 0.0 & 1.0 & 0.0 & 0.0 & 245.2 \\
\hline Distilleries & 0.0 & 0.0 & 0.0 & 0.0 & 0.0 & 0.0 & 0.0 & 0.0 & 0.0 & 0.0 & 0.0 & 0.0 & 0.0 & 0.0 & 0.0 & 103.6 & 0.0 & 0.0 & 0.0 & 0.0 & 103.6 \\
\hline Dog/cat food manufacturing & 0.0 & 0.0 & 0.0 & 0.0 & 0.0 & 0.0 & 0.0 & 0.0 & 0.0 & 0.0 & 0.0 & 5.4 & 0.0 & 0.0 & 0.0 & 0.0 & 0.0 & 0.0 & 0.0 & 0.0 & 5.4 \\
\hline Dry pasta manufacturing & 0.0 & 0.0 & 0.0 & 0.0 & 0.0 & 0.0 & 0.3 & 0.0 & 0.0 & 0.0 & 0.0 & 0.0 & 0.0 & 0.0 & 0.0 & 0.0 & 0.0 & 0.0 & 0.0 & 0.0 & 0.3 \\
\hline Flavoring syrup/concentrate mfg & 0.0 & 0.0 & 0.0 & 0.0 & 0.0 & 0.0 & 0.7 & 0.0 & 0.0 & 0.0 & 0.0 & 2.5 & 0.0 & 0.0 & 0.0 & 67.3 & 0.0 & 2.4 & 0.0 & 0.0 & 72.9 \\
\hline Mayonnaise/dressing/sauce mfg & 1.2 & 0.0 & 0.0 & 0.0 & 0.0 & 0.0 & 0.0 & 0.0 & 0.0 & 0.0 & 0.0 & 5.1 & 0.0 & 0.0 & 0.0 & 0.0 & 0.0 & 0.0 & 0.0 & 0.0 & 6.3 \\
\hline Mixes/dough purchased flour & 0.0 & 0.0 & 0.0 & 0.0 & 0.0 & 0.0 & 0.0 & 0.0 & 0.0 & 0.0 & 0.0 & 2.0 & 0.0 & 0.0 & 0.0 & 0.0 & 0.0 & 0.0 & 0.0 & 0.0 & 2.0 \\
\hline Other animal food manufacturing & 0.0 & 0.0 & 0.0 & 0.0 & 0.0 & 0.0 & 9.5 & 0.0 & 14.0 & 0.0 & 63.7 & 0.0 & 38.1 & 3.0 & 0.0 & 129.9 & 0.0 & 0.8 & 0.0 & 4.8 & 263.7 \\
\hline Other snack food manufacturing & 0.0 & 0.0 & 0.0 & 0.0 & 0.0 & 0.0 & 20.0 & 0.0 & 0.0 & 0.0 & 0.0 & 332.7 & 0.0 & 0.0 & 23.7 & 0.0 & 0.0 & 0.0 & 0.0 & 0.0 & 376.3 \\
\hline Roasted nuts/peanut butter mfg & 0.0 & 0.0 & 0.0 & 0.0 & 0.0 & 0.0 & 21.4 & 0.0 & 0.0 & 0.0 & 0.0 & 0.0 & 0.0 & 0.0 & 0.0 & 2.8 & 0.0 & 0.0 & 0.0 & 0.0 & 24.2 \\
\hline Soft drink \& ice manufacturing & 8.0 & 0.0 & 0.0 & 0.0 & 0.0 & 0.0 & $1,062.0$ & 0.0 & 32.5 & 0.0 & 0.0 & 526.1 & 10.3 & 102.6 & 14.7 & 11.4 & 4.5 & 0.0 & 4.3 & 0.0 & $1,776.2$ \\
\hline Spice \& extract manufacturing & 0.7 & 0.0 & 0.0 & 0.0 & 0.0 & 0.0 & 0.2 & 0.0 & 0.0 & 0.0 & 0.0 & 1.6 & 0.0 & 1.3 & 38.2 & 83.7 & 0.0 & 9.1 & 0.0 & 0.0 & 134.9 \\
\hline Wineries & 0.0 & 0.0 & 0.0 & 0.0 & 0.0 & 0.0 & 0.0 & 0.0 & 51.7 & 28.9 & 0.0 & 0.0 & 0.0 & 0.0 & 0.0 & 0.0 & 0.0 & 0.0 & 0.0 & 0.0 & 80.6 \\
\hline Total & 11.9 & 0.0 & 0.0 & 0.0 & 0.8 & 1.9 & $1,538.4$ & 21.8 & 101.3 & 85.2 & 65.4 & $1,031.4$ & 57.1 & 109.8 & 104.1 & 747.0 & 16.2 & 17.2 & 5.2 & 4.8 & $3,919.5$ \\
\hline \multicolumn{22}{|c|}{ Sugarcane Farming, Refined Sugar $\&$ Confections } \\
\hline Confectionery mfg & 1.3 & 0.0 & 0.0 & 0.0 & 0.0 & 0.0 & 3.4 & 0.0 & 0.0 & 0.0 & 0.0 & 10.5 & 0.0 & 0.0 & 2.7 & 1.8 & 3.6 & 0.0 & 2.0 & 0.0 & 25.3 \\
\hline Non-chocolate confectionery mfg & 0.0 & 0.0 & 0.0 & 0.0 & 0.0 & 0.0 & 115.8 & 0.0 & 0.0 & 11.5 & 0.0 & 0.0 & 0.0 & 0.0 & 30.1 & 25.4 & 0.0 & 19.4 & 0.0 & 0.0 & 202.2 \\
\hline Total & 1.3 & 0.0 & 0.0 & 0.0 & 0.0 & 0.0 & 119.2 & 0.0 & 0.0 & 11.5 & 0.0 & 10.5 & 0.0 & 0.0 & 32.7 & 27.2 & 3.6 & 19.4 & 2.0 & 0.0 & 227.5 \\
\hline Tobacco Farming \& Mfg & 2.0 & 0.0 & 0.0 & 0.0 & 1.7 & 0.0 & 397.2 & 0.0 & 0.0 & 0.0 & 0.0 & 0.3 & 0.0 & 0.0 & 0.1 & 0.0 & 1.0 & 0.6 & 0.0 & 0.0 & 403.0 \\
\hline Wildlife (hunting) & 0.0 & 0.0 & 4.3 & 0.0 & 0.0 & 0.0 & 3.1 & 13.7 & 0.0 & 0.0 & 0.0 & 4.5 & 0.0 & 2.7 & 12.3 & 0.0 & 0.0 & 0.5 & 0.8 & 0.0 & 41.9 \\
\hline Grand Total & 389.9 & 128.1 & 451.8 & 392.5 & 195.1 & 686.2 & $6,766.4$ & 618.0 & 957.8 & $2,684.4$ & 313.7 & $3,172.6$ & 288.4 & 633.9 & $1,529.0$ & $6,656.4$ & 411.3 & 426.9 & 724.3 & 255.7 & $27,682.5$ \\
\hline
\end{tabular}


Table 10. Employment impacts of agriculture and natural resource industries, central region of Florida, 2002 data

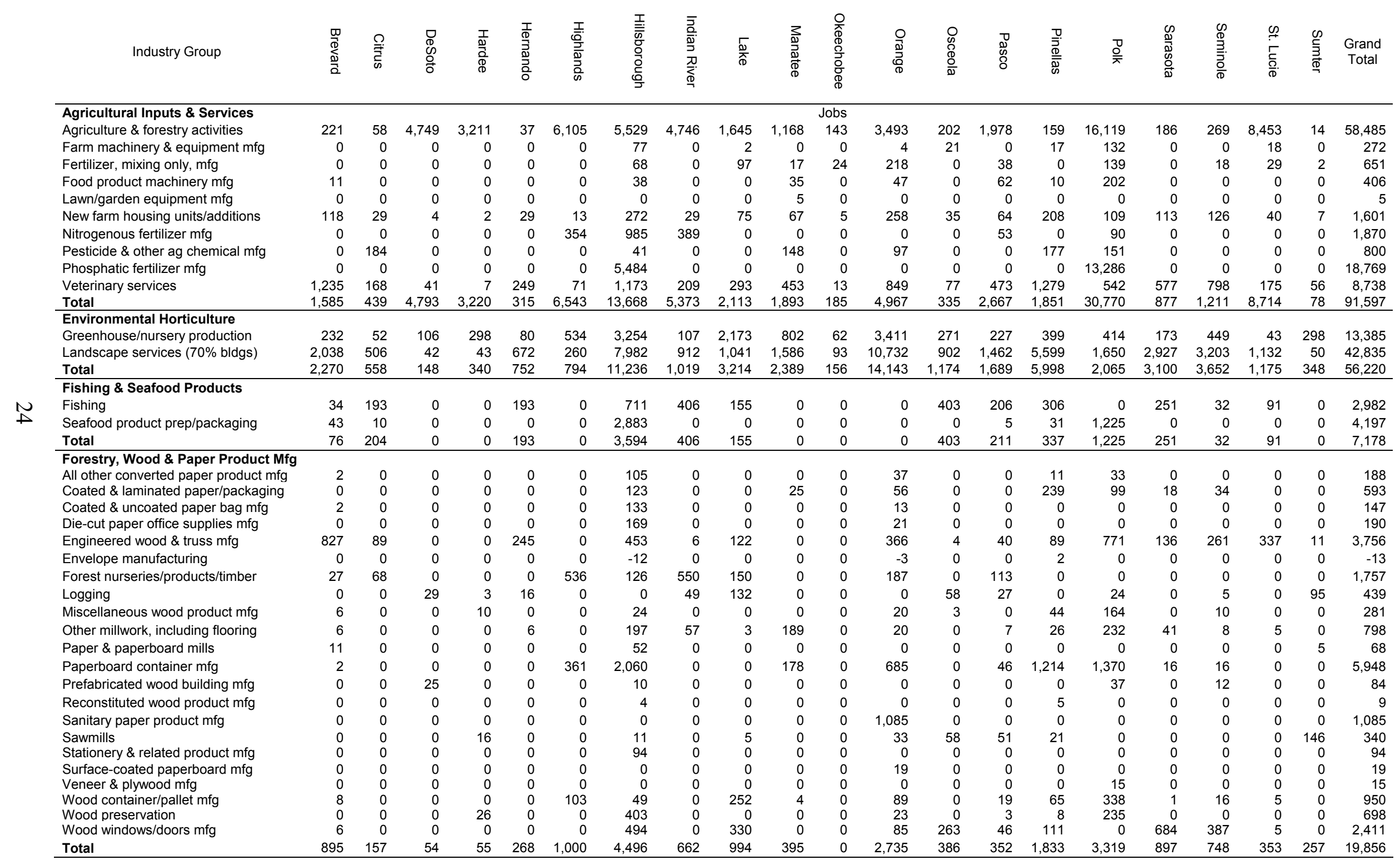


Table 10 (continued). Employment impacts of agriculture and natural resource industries, central region of Florida, 2002 data

\begin{tabular}{|c|c|c|c|c|c|c|c|c|c|c|c|c|c|c|c|c|c|c|c|c|c|}
\hline Industry Group & 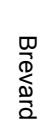 & $\frac{\rho}{\vec{f}}$ & $\begin{array}{l}0 \\
\mathbb{0} \\
0 \\
0 \\
0 \\
0\end{array}$ & $\begin{array}{l}\mathrm{T} \\
\frac{\mathbf{D}}{2} \\
\overline{0} \\
0\end{array}$ & $\begin{array}{l}\text { T } \\
\text { D } \\
3 \\
0 \\
0 \\
\text { J } \\
0\end{array}$ & 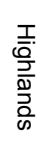 & $\begin{array}{l}\text { I } \\
\overline{\bar{D}} \\
\overline{0} \\
\stackrel{0}{0} \\
\end{array}$ & 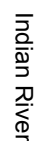 & $\frac{\overline{2}}{\hat{\hat{\alpha}}}$ & 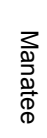 & 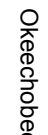 & 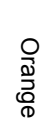 & $\begin{array}{l}\text { O } \\
\text { N } \\
\text { D } \\
\frac{0}{N}\end{array}$ & 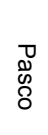 & 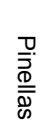 & $\frac{0}{2}$ & 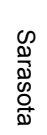 & 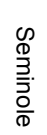 & 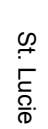 & 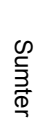 & $\begin{array}{c}\text { Grand } \\
\text { Total }\end{array}$ \\
\hline
\end{tabular}

\section{Fruit \& Vegetable Farming \& Processing}

Frozen food manufacturng

Fruit \& vegetable canning/drying

Fruit farming

Vegetable \& melon farming

Total

Grain \& Oilseed Farming \& Processing

Flour milling

Grain farming

Total

\begin{tabular}{|c|c|c|c|c|c|c|c|c|c|c|c|c|c|c|c|c|c|c|c|c|}
\hline \multicolumn{21}{|c|}{ Jobs } \\
\hline 0 & 0 & 470 & 0 & 11 & 296 & 561 & 331 & 161 & 0 & 0 & 1,657 & 0 & 1,264 & 0 & 7,587 & 0 & 0 & 156 & 0 & 12,495 \\
\hline 0 & 0 & 0 & 221 & 0 & 63 & 209 & 0 & 1,505 & 10,380 & 0 & 123 & 36 & 0 & 1,605 & 1,811 & 15 & 0 & 1,444 & 0 & 17,413 \\
\hline 366 & 92 & 2,947 & 2,364 & 64 & 3,469 & 9,116 & 2,160 & 941 & 1,463 & 602 & 477 & 485 & 925 & 24 & 8,563 & 246 & 83 & 1,859 & 28 & 36,272 \\
\hline 43 & 6 & 74 & 316 & 118 & 62 & 3,324 & 45 & 10 & 4,861 & 364 & 20 & 101 & 113 & 19 & 59 & 92 & 1 & 9 & 93 & 9,730 \\
\hline 408 & 98 & 3,491 & 2,901 & 192 & 3,891 & 13,210 & 2,536 & 2,616 & 16,705 & 966 & 2,277 & 622 & 2,301 & 1,648 & 18,020 & 354 & 85 & 3,468 & 121 & 75,911 \\
\hline
\end{tabular}

\section{Livestock \& Dairy Farming \& Animal Products Mfg}

Animal production, except cattle \& poultry

axcept poultry, slaughtering

$908 \quad 3$

Cattle ranching \& farming

N Cheese manufacturing

Creamery butter manufacturing

Dry/condensed/evaporated dairy products

Fluid milk manufacturing

Ice cream \& frozen dessert mfg

Meat processed from carcasses

Poultry \& egg production

Poultry processing

Rendering \& meat byproduct processing

Total

Mining

Drilling oil \& gas wells

Oil \& gas extraction

Other nonmetallic mineral mining

Sand/gravel/clay/refractory mining

Stone mining \& quarrying

Support activities for oil \& gas operations

Support activities for other mining

Total

\begin{tabular}{rrrrrrrrrrrrrrrrrrrrrrr}
4 & 0 & 0 & 0 & 0 & 7 & 22 & 0 & 5 & 7 & 5 & 11 & 0 & 0 & 0 & 31 & 67 & 8 & 0 & 0 & 167 \\
0 & 0 & 0 & 0 & 0 & 0 & 125 & 0 & 0 & 0 & 0 & 27 & 0 & 26 & 1,779 & 0 & 0 & 80 & 119 & 0 & 2,156 \\
4 & 4 & 0 & 220 & 0 & 123 & 146 & 23 & 544 & 1 & 0 & 14 & 0 & 3 & 0 & 3,845 & 0 & 0 & 0 & 0 & 4,925 \\
0 & 12 & 0 & 0 & 17 & 0 & 36 & 47 & 179 & 5 & 4 & 46 & 0 & 0 & 107 & 448 & 0 & 0 & 23 & 0 & 925 \\
83 & 71 & 0 & 0 & 628 & 0 & 57 & 0 & 8 & 0 & 0 & 0 & 0 & 58 & 0 & 298 & 49 & 0 & 27 & 147 & 1,426 \\
11 & 0 & 0 & 0 & 0 & 0 & 8 & 0 & 4 & 0 & 0 & 2 & 0 & 1 & 0 & 0 & 0 & 4 & 0 & 0 & 30 \\
0 & 0 & 0 & 0 & 0 & 0 & 0 & 0 & 0 & 0 & 0 & 0 & 0 & 0 & 30 & 0 & 0 & 0 & 0 & 0 & 30 \\
101 & 87 & 0 & 220 & 645 & 130 & 393 & 70 & 739 & 13 & 9 & 100 & 0 & 89 & 1,916 & 4,622 & 117 & 93 & 169 & 147 & 9,659 \\
\hline
\end{tabular}


Table 10 (continued). Employment impacts of agriculture and natural resource industries, central region of Florida, 2002 data

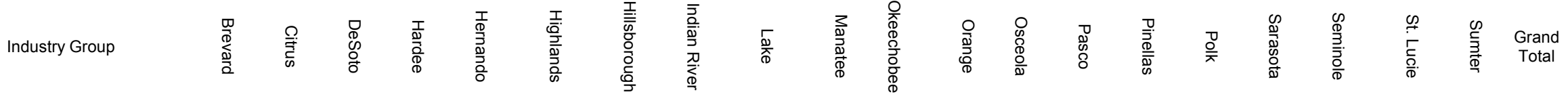

\begin{tabular}{|c|c|c|c|c|c|c|c|c|c|c|c|c|c|c|c|c|c|c|c|c|c|}
\hline \multirow[b]{2}{*}{ Other Crop Farming } & \multicolumn{21}{|c|}{ Jobs } \\
\hline & 2 & 6 & 7 & 10 & 25 & 13 & 32 & 0 & 14 & 18 & 11 & 4 & 6 & 39 & 0 & 18 & 2 & 2 & 0 & 53 & 261 \\
\hline \multicolumn{22}{|l|}{ Other Food Product Manufacturing } \\
\hline All other food manufacturing & 0 & 0 & 0 & 0 & 0 & 0 & 5 & 104 & 0 & 0 & 0 & 7 & 2 & 0 & 40 & 0 & 4 & 7 & 1 & 0 & 170 \\
\hline Bread/bakery, except frozen, mfg & 30 & 0 & 0 & 0 & 7 & 15 & 696 & 26 & 42 & 467 & 16 & 1,338 & 59 & 33 & 153 & 2,592 & 140 & 29 & 8 & 0 & 5,653 \\
\hline Breweries & 0 & 0 & 0 & 0 & 0 & 0 & 561 & 0 & 6 & 0 & 0 & 0 & 0 & 0 & 0 & 580 & 0 & 0 & 0 & 0 & 1,147 \\
\hline Coffee \& tea manufacturing & 0 & 0 & 0 & 0 & 0 & 0 & 94 & 0 & 0 & 0 & 0 & 5 & 0 & 0 & 8 & 0 & 0 & 0 & 0 & 0 & 107 \\
\hline Cookie \& cracker manufacturing & 0 & 0 & 0 & 0 & 0 & 0 & 1,509 & 0 & 0 & 5 & 0 & 4 & 0 & 0 & 0 & 10 & 0 & 5 & 0 & 0 & 1,534 \\
\hline Distilleries & 0 & 0 & 0 & 0 & 0 & 0 & 0 & 0 & 0 & 0 & 0 & 0 & 0 & 0 & 0 & 1,001 & 0 & 0 & 0 & 0 & 1,001 \\
\hline Dog/cat food manufacturing & 0 & 0 & 0 & 0 & 0 & 0 & 0 & 0 & 0 & 0 & 0 & 26 & 0 & 0 & 0 & 0 & 0 & 0 & 0 & 0 & 26 \\
\hline Dry pasta manufacturing & 0 & 0 & 0 & 0 & 0 & 0 & 3 & 0 & 0 & 0 & 0 & 0 & 0 & 0 & 0 & 0 & 0 & 0 & 0 & 0 & 3 \\
\hline Flavoring syrup/concentrate mfg & 0 & 0 & 0 & 0 & 0 & 0 & 3 & 0 & 0 & 0 & 0 & 14 & 0 & 0 & 0 & 407 & 0 & 15 & 0 & 0 & 438 \\
\hline Mayonnaise/dressing/sauce mfg & 4 & 0 & 0 & 0 & 0 & 0 & 0 & 0 & 0 & 0 & 0 & 15 & 0 & 0 & 0 & 0 & 0 & 0 & 0 & 0 & 18 \\
\hline Mixes/dough purchased flour & 0 & 0 & 0 & 0 & 0 & 0 & 0 & 0 & 0 & 0 & 0 & 6 & 0 & 0 & 0 & 0 & 0 & 0 & 0 & 0 & 6 \\
\hline Other animal food manufacturing & 0 & 0 & 0 & 0 & 0 & 0 & 56 & 0 & 66 & 0 & 275 & 0 & 181 & 13 & 0 & 714 & 0 & 4 & 0 & 19 & 1,328 \\
\hline Other snack food manufacturing & 0 & 0 & 0 & 0 & 0 & 0 & 75 & 0 & 0 & 0 & 0 & 1,932 & 0 & 0 & 67 & 0 & 0 & 0 & 0 & 0 & 2,074 \\
\hline Roasted nuts/peanut butter mfg & 0 & 0 & 0 & 0 & 0 & 0 & 124 & 0 & 0 & 0 & 0 & 0 & 0 & 0 & 0 & 17 & 0 & 0 & 0 & 0 & 141 \\
\hline Soft drink \& ice manufacturing & 51 & 0 & 0 & 0 & 0 & 0 & 6,592 & 0 & 216 & 0 & 0 & 3,173 & 69 & 623 & 107 & 84 & 27 & 0 & 34 & 0 & 10,976 \\
\hline Spice \& extract manufacturing & 3 & 0 & 0 & 0 & 0 & 0 & 1 & 0 & 0 & 0 & 0 & 7 & 0 & 5 & 187 & 531 & 0 & 41 & 0 & 0 & 777 \\
\hline Wineries & 0 & 0 & 0 & 0 & 0 & 0 & 0 & 0 & 402 & 205 & 0 & 0 & 0 & 0 & 0 & 0 & 0 & 0 & 0 & 0 & 607 \\
\hline Total & 89 & 0 & 0 & 0 & 7 & 15 & 9,718 & 131 & 732 & 677 & 291 & 6,528 & 311 & 674 & 563 & 5,938 & 171 & 100 & 43 & 19 & 26,007 \\
\hline \multicolumn{22}{|c|}{ Sugarcane Farming, Refined Sugar \& Confections } \\
\hline Confectionery $\mathrm{mfg}$ & 11 & 0 & 0 & 0 & 0 & 0 & 33 & 0 & 0 & 0 & 0 & 79 & 0 & 0 & 23 & 16 & 27 & 0 & 20 & 0 & 210 \\
\hline Non-chocolate confectionery $\mathrm{mfg}$ & 0 & 0 & 0 & 0 & 0 & 0 & 859 & 0 & 0 & 98 & 0 & 0 & 0 & 0 & 238 & 206 & 0 & 155 & 0 & 0 & 1,556 \\
\hline Total & 11 & 0 & 0 & 0 & 0 & 0 & 891 & 0 & 0 & 98 & 0 & 79 & 0 & 0 & 261 & 222 & 27 & 155 & 20 & 0 & 1,765 \\
\hline Tobacco Farming \& Mfg & 22 & 0 & 0 & 0 & 6 & 0 & 2,665 & 0 & 0 & 0 & 0 & 3 & 0 & 0 & 1 & 0 & 3 & 4 & 0 & 0 & 2,704 \\
\hline Wildlife (hunting) & 0 & 0 & 91 & 0 & 0 & 0 & 42 & 237 & 0 & 0 & 0 & 65 & 0 & 70 & 93 & 0 & 0 & 7 & 11 & 0 & 617 \\
\hline Grand Total & 5,634 & 1,795 & 8,855 & 7,203 & 2,671 & 12,715 & 63,564 & 10,500 & 11,198 & 22,839 & 2,744 & 32,145 & 3,652 & 8,807 & 16,201 & 67,373 & 6,015 & 6,172 & 14,082 & 2,233 & 306,398 \\
\hline
\end{tabular}

Source: IMPLAN data for Florida counties (2005) 
Table 11. Value-added impacts of agriculture and natural resource industries, central region of Florida, 2002 data

Industry Group

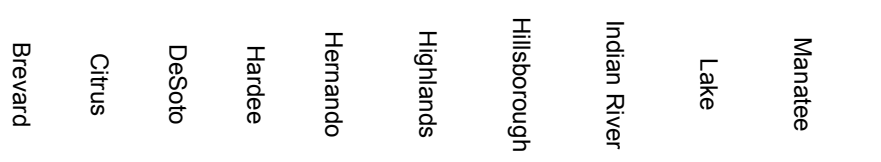

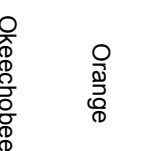

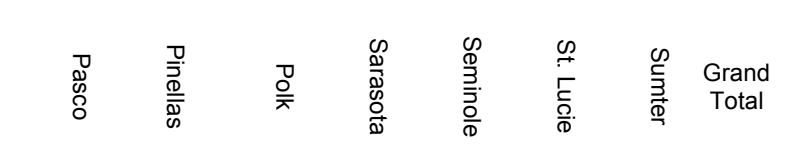

Agricultural Inputs \& Services

Farm machinery \& equipment $\mathrm{mfg}$

Fertilizer, mixing only, mfg

Food product machinery $\mathrm{mfg}$

Lawn/garden equipment $\mathrm{mfg}$

New farm housing units/additions

Nitrogenous fertilizer $\mathrm{mfg}$

Pesticide \& other ag chemical mfg

Phosphatic fertilizer $\mathrm{mfg}$

Veterinary services

$\begin{array}{rrrrrrrrrr}19.1 & 3.2 & 72.3 & 45.9 & 0.7 & 93.8 & 141.5 & 113.1 & 31.7 & 14.8 \\ 0.0 & 0.0 & 0.0 & 0.0 & 0.0 & 0.0 & 5.0 & 0.0 & 0.1 & 0.0 \\ 0.0 & 0.0 & 0.0 & 0.0 & 0.0 & 0.0 & 6.7 & 0.0 & 6.4 & 1.6 \\ 0.5 & 0.0 & 0.0 & 0.0 & 0.0 & 0.0 & 2.1 & 0.0 & 0.0 & 2.0 \\ 0.0 & 0.0 & 0.0 & 0.0 & 0.0 & 0.0 & 0.0 & 0.0 & 0.0 & 0.1 \\ 4.5 & 0.9 & 0.1 & 0.1 & 0.7 & 0.3 & 12.3 & 1.2 & 2.8 & 2.8 \\ 0.0 & 0.0 & 0.0 & 0.0 & 0.0 & 24.2 & 74.1 & 24.0 & 0.0 & 0.0 \\ 0.0 & 17.5 & 0.0 & 0.0 & 0.0 & 0.0 & 3.4 & 0.0 & 0.0 & 10.6 \\ 0.0 & 0.0 & 0.0 & 0.0 & 0.0 & 0.0 & 373.1 & 0.0 & 0.0 & 0.0 \\ 42.1 & 3.6 & 0.8 & 0.1 & 6.0 & 1.3 & 36.8 & 5.2 & 7.5 & 20.8\end{array}$

5 (\$Million)

$\begin{array}{rrrr}1.5 & 74.2 & 4.5 & 22.5 \\ 0.0 & 0.2 & 1.0 & 0.0\end{array}$

$\begin{array}{lllllll}6.7 & 182.1 & 10.0 & 5.0 & 146.6 & 0.1 & 989.3\end{array}$

Total

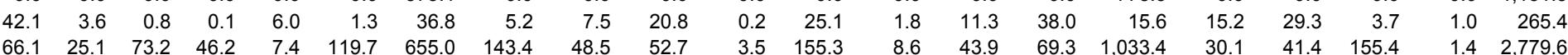

\section{Environmental Horticulture}

Landscape services $(70 \%$ bldgs)

Fishing \& Seafood Products

N Fishing

Seafood product prep/packaging

$\begin{array}{rrrrrrrrrrrrrrrrrrrrrr}10.6 & 1.7 & 3.1 & 21.1 & 2.4 & 37.0 & 187.0 & 7.8 & 130.0 & 46.5 & 6.1 & 207.1 & 17.9 & 10.3 & 19.6 & 29.3 & 10.6 & 24.5 & 3.2 & 11.4 & 787.1 \\ 5 & 12.6 & 1.1 & 1.0 & 18.1 & 5.6 & 21.9 & 26.3 & 28.5 & 49.7 & 2.4 & 335.4 & 22.4 & 37.2 & 179.9 & 41.6 & 83.7 & 92.9 & 31.7 & 0.7 & 1.234 .2\end{array}$

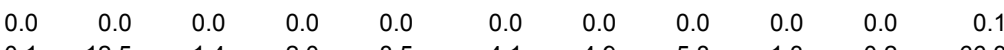

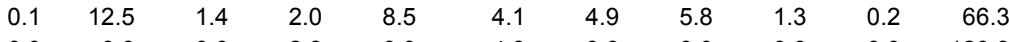

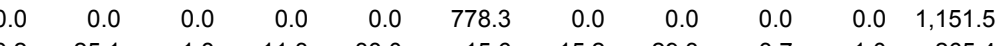

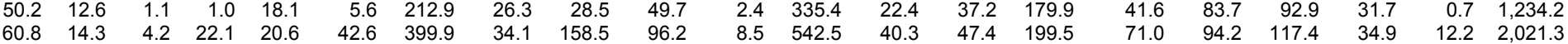
$\begin{array}{rrrrrrrrrrrrrrrrrrrrr}0.6 & 3.5 & 0.0 & 0.0 & 3.6 & 0.0 & 24.1 & 8.8 & 1.5 & 0.0 & 0.0 & 0.0 & 10.0 & 1.0 & 13.9 & 0.0 & 3.8 & 0.7 & 2.1 & 0.0 & 73.5 \\ 0.8 & 0.3 & 0.0 & 0.0 & 0.0 & 0.0 & 146.0 & 0.0 & 0.0 & 0.0 & 0.0 & 0.0 & 0.0 & 0.1 & 1.0 & 57.7 & 0.0 & 0.0 & 0.0 & 0.0 & 206.1\end{array}$

Forestry, Wood \& Paper Product Mfg

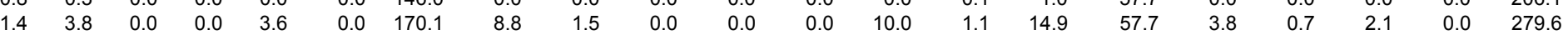

All other converted paper product $\mathrm{mfg}$

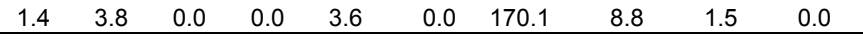

Coated \& laminated paper/packaging

$\begin{array}{lllll}0.0 & 0.0 & 0.0 & 0.0 & 0.0 \\ 0.0 & 0.0 & 0.0 & 0.0 & 0.0\end{array}$

作

-cut paper office supplies $\mathrm{m}$

Engineered wood \& truss $\mathrm{mfg}$

Envelope manufacturing

Forest nurseries/products/timber

Logging

Miscellaneous wood product mfg

Other millwork, including flooring

Paper \& paperboard mills

Paperboard container $\mathrm{mfg}$

Prefabricated wood building $\mathrm{mfg}$

Reconstituted wood product mfg

Sanitary paper product $\mathrm{mfg}$

Sawmills

Stationery \& related product $\mathrm{mfg}$

Surface-coated paperboard $\mathrm{mfg}$

Veneer \& plywood mfg

Wood container/pallet $\mathrm{mfg}$

Wood preservation

Wood windows/doors $\mathrm{mfg}$

$\begin{array}{lllll}0.0 & 0.0 & 0.0 & 0.0 & 0.0 \\ 0.0 & 0.0 & 0.0 & 0.0 & 0.0 \\ 0.0 & 0.0 & 0.0 & 0.0 & 0.0\end{array}$

$\begin{array}{lllllllllll}0.0 & 0.0 & 0.0 & 0.0 & 0.0 & 0.0 & 5.4 & 0.0 & 0.0 & 1.4\end{array}$

$\begin{array}{rrrrrrrrrr}0.0 & 0.0 & 0.0 & 0.0 & 0.0 & 0.0 & 11.3 & 0.0 & 0.0 & 0.0\end{array}$

$\begin{array}{llllllllll}43.3 & 3.7 & 0.0 & 0.0 & 12.3 & 0.0 & 23.4 & 0.3 & 5.2 & 0.0\end{array}$

$\begin{array}{rrrrrrrrrr}0.0 & 0.0 & 0.0 & 0.0 & 0.0 & 0.0 & -9.0 & 0.0 & 0.0 & 0.0\end{array}$

$\begin{array}{lll}11.8 & 5.0 & 18.1\end{array}$

$\begin{array}{llllllllll}0.0 & 0.0 & 4.1 & 0.2 & 1.0 & 0.0 & 0.0 & 2.4 & 6.3 & 0.0\end{array}$

$\begin{array}{llllllllll}0.2 & 0.0 & 0.0 & 0.5 & 0.0 & 0.0 & 0.7 & 0.0 & 0.0 & 0.0 \\ 0.2 & 0.0 & 0.0 & 0.0 & 0.2 & 0.0 & 7.4 & 1.9 & 0.1 & 8.3\end{array}$

$\begin{array}{llllllllll}0.2 & 0.0 & 0.0 & 0.0 & 0.2 & 0.0 & 7.4 & 1.9 & 0.1 & 8.3 \\ 0.5 & 0.0 & 0.0 & 0.0 & 0.0 & 0.0 & 3.5 & 0.0 & 0.0 & 0.0\end{array}$

$\begin{array}{rrrrrrrrrr}0.0 & 0.0 & 0.0 & 0.0 & 0.0 & 19.2 & 125.3 & 0.0 & 0.0 & 10.2\end{array}$

$\begin{array}{llllllllll}0.0 & 0.0 & 2.1 & 0.0 & 0.0 & 0.0 & 0.6 & 0.0 & 0.0 & 0.0 \\ 0.0 & 0.0 & 0.0 & 0.0 & 0.0 & 0.0 & 0.8 & 0.0 & 0.0 & 0.0\end{array}$

$\begin{array}{llllllllllll}0.0 & 0.0 & 0.0 & 0.0 & 0.0 & 0.0 & 0.0 & 0.0 & 0.0 & 0.0 & 0.0 & 99.1\end{array}$

$\begin{array}{lllllllllllllll}0.0 & 0.0 & 0.0 & 0.7 & 0.0 & 0.0 & 0.5 & 0.0 & 0.1 & 0.0 & 0.0 & 2.3 & 2.8 & 0.0 & 0.0\end{array}$

$\begin{array}{llllllllllllllll}0.0 & 0.0 & 0.0 & 0.0 & 0.0 & 0.0 & 5.7 & 0.0 & 0.0 & 0.0 & 0.0 & 0.0 & 0.0 & 0.0 & 0.0\end{array}$

$\begin{array}{llllllllll}0.0 & 0.0 & 0.0 & 0.0 & 0.0 & 0.0 & 0.0 & 0.0 & 0.0 & 0.0\end{array}$

$\begin{array}{llllllllll}0.0 & 0.0 & 0.0 & 0.0 & 0.0 & 0.0 & 0.0 & 0.0 & 0.0 & 0.0\end{array}$

$\begin{array}{lllllllllllll}0.3 & 0.0 & 0.0 & 0.0 & 0.0 & 4.2 & 2.2 & 0.0 & 8.9 & 0.0 & 0.0 & 0.0 & 0.0\end{array}$

$\begin{array}{llllllllll}0.0 & 0.0 & 0.0 & 1.3 & 0.0 & 0.0 & 23.5 & 0.0 & 0.0 & 0.0\end{array}$

$\begin{array}{rrrrrrrrrr}0.3 & 0.0 & 0.0 & 0.0 & 0.0 & 0.0 & 28.6 & 0.0 & 15.5 & 0.0 \\ 46.6 & 7.3 & 6.2 & 2.6 & 13.4 & 35.2 & 249.6 & 22.6 & 41.2 & 20.0\end{array}$

$\begin{array}{lllll}0.0 & 2.9 & 0.0 & 0.6 & 2.3 \\ 0.0 & 1.8 & 0.0 & 0.1 & 0.4\end{array}$

$\begin{array}{rrrrr}0.0 & 5.0 & 12.4 & 2.1 & 6.4 \\ 0.0 & 193.7 & 19.8 & 12.3 & 9.3\end{array}$

Total

\begin{tabular}{rrrrrrr}
0.7 & 1.8 & 0.0 & 0.0 & 0.0 & 0.0 & 12.4 \\
4.4 & 5.9 & 1.0 & 2.4 & 0.0 & 0.0 & 35.6 \\
0.0 & 0.0 & 0.0 & 0.0 & 0.0 & 0.0 & 5.8 \\
0.0 & 0.0 & 0.0 & 0.0 & 0.0 & 0.0 & 12.6 \\
4.9 & 43.0 & 6.7 & 14.2 & 17.6 & 0.6 & 198.0 \\
0.0 & 0.0 & 0.0 & 0.0 & 0.0 & 0.0 & -10.8 \\
0.0 & 0.0 & 0.0 & 0.0 & 0.0 & 0.0 & 54.0 \\
0.0 & 1.8 & 0.0 & 0.3 & 0.0 & 1.6 & 23.4 \\
1.7 & 7.4 & 0.0 & 0.4 & 0.0 & 0.0 & 12.3 \\
1.0 & 9.8 & 1.6 & 0.3 & 0.1 & 0.0 & 32.0 \\
0.0 & 0.0 & 0.0 & 0.0 & 0.0 & 0.2 & 4.2 \\
4.5 & 81.3 & 1.0 & 0.8 & 0.0 & 0.0 & 350.7 \\
0.0 & 2.0 & 0.0 & 0.7 & 0.0 & 0.0 & 5.3 \\
0.3 & 0.0 & 0.0 & 0.0 & 0.0 & 0.0 & 1.0 \\
0.0 & 0.0 & 0.0 & 0.0 & 0.0 & 0.0 & 99.1 \\
1.3 & 0.0 & 0.0 & 0.0 & 0.0 & 6.1 & 15.9 \\
0.0 & 0.0 & 0.0 & 0.0 & 0.0 & 0.0 & 5.7 \\
0.0 & 0.0 & 0.0 & 0.0 & 0.0 & 0.0 & 0.9 \\
0.0 & 1.0 & 0.0 & 0.0 & 0.0 & 0.0 & 1.0 \\
2.3 & 14.7 & 0.0 & 0.5 & 0.1 & 0.0 & 36.9 \\
0.4 & 12.5 & 0.0 & 0.0 & 0.0 & 0.0 & 39.5 \\
6.9 & 0.0 & 40.8 & 23.9 & 0.2 & 0.0 & 135.7 \\
8.3 & 181.3 & 51.2 & 43.4 & 18.1 & 8.4 & $1,071.2$ \\
\hline & & & & & &
\end{tabular}


Table 11 (continued). Value-added impacts of agriculture and natural resource industries, central region of Florida, 2002 data

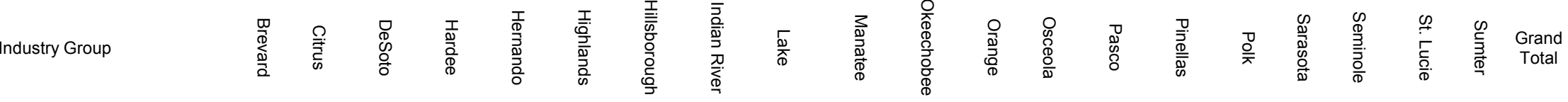

\begin{tabular}{|c|c|c|c|c|c|c|c|c|c|c|c|c|c|c|c|c|c|c|c|c|c|}
\hline \multicolumn{11}{|l|}{ Fruit \& Vegetable Farming \& Processing } & \multicolumn{11}{|c|}{ (\$Million) } \\
\hline Frozen food manufacturing & 0.0 & 0.0 & 35.4 & 0.0 & 0.8 & 21.7 & 40.1 & 21.5 & 8.6 & 0.0 & 0.0 & 131.1 & 0.0 & 78.9 & 0.0 & 501.5 & 0.0 & 0.0 & 8.3 & 0.0 & 847.7 \\
\hline Fruit \& vegetable canning/drying & 0.0 & 0.0 & 0.0 & 15.3 & 0.0 & 5.3 & 14.6 & 0.0 & 98.3 & 893.1 & 0.0 & 8.7 & 3.1 & 0.0 & 118.6 & 116.9 & 1.0 & 0.0 & 91.9 & 0.0 & $1,366.8$ \\
\hline Fruit farming & 9.5 & 1.6 & 95.3 & 73.9 & 0.9 & 122.5 & 340.1 & 86.8 & 31.4 & 49.1 & 36.6 & 18.2 & 19.0 & 21.0 & 0.8 & 275.9 & 8.9 & 2.5 & 60.9 & 0.5 & $1,255.5$ \\
\hline Vegetable \& melon farming & 2.0 & 0.3 & 4.0 & 18.6 & 4.4 & 4.0 & 179.0 & 3.0 & 0.7 & 245.8 & 29.5 & 1.0 & 6.8 & 4.8 & 0.9 & 4.7 & 5.8 & 0.1 & 0.6 & 3.8 & 519.8 \\
\hline Total & 11.5 & 1.8 & 134.6 & 107.8 & 6.1 & 153.5 & 573.8 & 111.3 & 139.0 & $1,188.0$ & 66.1 & 159.0 & 28.9 & 104.8 & 120.3 & 899.0 & 15.7 & 2.6 & 161.7 & 4.3 & $3,989.7$ \\
\hline \multicolumn{22}{|l|}{ Grain \& Oilseed Farming \& Processing } \\
\hline Flour milling & 0.0 & 0.0 & 0.0 & 0.0 & 0.0 & 0.0 & 27.3 & 0.0 & 0.0 & 0.0 & 0.0 & 0.0 & 0.0 & 0.0 & 0.0 & 1.2 & 0.0 & 0.0 & 0.0 & 0.0 & 28.5 \\
\hline Grain farming & 0.0 & 0.0 & 0.0 & 0.0 & 0.0 & 0.0 & 0.0 & 0.0 & 0.0 & 0.0 & 0.0 & 0.0 & 0.0 & 0.0 & 0.0 & 0.0 & 0.0 & 0.0 & 0.0 & 0.0 & 0.0 \\
\hline Total & 0.0 & 0.0 & 0.0 & 0.0 & 0.0 & 0.0 & 27.4 & 0.0 & 0.0 & 0.0 & 0.0 & 0.0 & 0.0 & 0.0 & 0.0 & 1.2 & 0.0 & 0.0 & 0.0 & 0.0 & 28.6 \\
\hline \multicolumn{22}{|c|}{ Livestock \& Dairy Farming \& Animal Products Mfg } \\
\hline Anima & 0.4 & 0.5 & 0.1 & 0.1 & 0.2 & 0.2 & 11.6 & 0.2 & 4.5 & 2.3 & 1.9 & 1.3 & 1.0 & 0.4 & 0.2 & 1.9 & 0.6 & 0.2 & 0.1 & 0.4 & 28.0 \\
\hline Animal, except poultry, slaughtering & 0.0 & 0.0 & 0.6 & 0.0 & 0.0 & 0.0 & 6.1 & 0.0 & 0.0 & 0.0 & 0.0 & 0.7 & 0.0 & 0.7 & 2.8 & 0.0 & 0.2 & 0.8 & 0.0 & 23.6 & 35.7 \\
\hline Cattle ranching \& farming & 1.9 & 2.1 & 2.6 & 6.3 & 2.8 & 5.8 & 9.8 & 1.1 & 2.9 & 10.4 & 32.9 & 0.8 & 7.3 & 8.2 & 0.0 & 2.3 & 2.6 & 0.8 & 0.4 & 1.0 & 102.0 \\
\hline Cheese manufacturing & 0.0 & 0.0 & 0.0 & 0.0 & 0.0 & 0.0 & 2.1 & 0.0 & 0.0 & 0.0 & 0.0 & 1.0 & 0.0 & 0.0 & 0.0 & 0.0 & 0.0 & 0.2 & 0.0 & 0.0 & 3.2 \\
\hline Creamery butter manufacturing & 0.0 & 0.0 & 0.0 & 0.0 & 0.0 & 0.0 & 0.0 & 0.0 & 0.0 & 0.0 & 0.0 & 0.0 & 0.0 & 0.0 & 0.0 & 0.0 & 0.0 & 0.0 & 0.0 & 0.0 & 0.0 \\
\hline Dry/condensed/evaporated dairy products & 0.0 & 0.0 & 0.0 & 0.0 & 0.0 & 0.0 & 0.0 & 0.0 & 0.0 & 0.0 & 0.0 & 0.0 & 0.0 & 0.0 & 4.4 & 0.0 & 0.0 & 0.0 & 0.0 & 0.0 & 4.4 \\
\hline Fluid milk manufacturing & 0.1 & 0.0 & 0.0 & 0.0 & 0.0 & 0.0 & 11.9 & 0.0 & 0.0 & 0.0 & 0.0 & 70.7 & 0.0 & 0.0 & 0.0 & 14.8 & 0.0 & 0.0 & 0.0 & 0.0 & 97.5 \\
\hline Ice cream \& frozen dessert mfg & 0.0 & 0.0 & 0.0 & 0.0 & 0.0 & 0.0 & 0.8 & 0.0 & 0.0 & 0.0 & 0.0 & 0.0 & 0.0 & 0.0 & 109.4 & 0.0 & 0.5 & 0.0 & 0.0 & 0.0 & 110.8 \\
\hline Meat processed from carcasses & 0.0 & 0.0 & 0.0 & 0.0 & 0.0 & 0.0 & 45.6 & 0.0 & 0.0 & 0.0 & 0.0 & 0.0 & 0.0 & 0.0 & 0.9 & 4.5 & 0.1 & 0.0 & 0.0 & 0.0 & 51.0 \\
\hline Poultry \& egg production & 0.0 & 0.0 & 0.5 & 0.0 & 2.3 & 0.0 & 18.4 & 0.0 & 6.6 & 0.0 & 0.0 & 0.0 & 0.3 & 5.8 & 0.0 & 9.2 & 2.1 & 0.0 & 0.0 & 3.4 & 48.6 \\
\hline Poultry processing & 0.0 & 0.0 & 0.0 & 0.0 & 0.0 & 0.0 & 0.1 & 0.0 & 0.0 & 0.0 & 0.0 & 0.0 & 0.0 & 0.0 & 0.0 & 0.0 & 0.0 & 0.0 & 0.0 & 0.0 & 0.1 \\
\hline Rendering \& meat byproduct processing & 0.0 & 0.0 & 0.0 & 0.0 & 0.0 & 0.0 & 16.0 & 0.0 & 0.0 & 0.0 & 0.0 & 0.7 & 0.0 & 0.0 & 0.0 & 0.0 & 0.0 & 0.0 & 0.0 & 0.0 & 16.7 \\
\hline Total & 2.4 & 2.6 & 3.9 & 6.4 & 5.3 & 6.0 & 122.5 & 1.3 & 14.0 & 12.7 & 34.8 & 75.1 & 8.7 & 15.1 & 117.7 & 32.7 & 6.1 & 1.9 & 0.5 & 28.5 & 498.1 \\
\hline \multicolumn{22}{|l|}{ Mining } \\
\hline Drilling oil \& gas wells & 0.1 & 0.0 & 0.0 & 0.0 & 0.0 & 0.1 & 1.2 & 0.0 & 0.1 & 0.3 & 0.1 & 0.6 & 0.0 & 0.0 & 0.0 & 1.6 & 2.5 & 0.4 & 0.0 & 0.0 & 7.1 \\
\hline Oil \& gas extraction & 0.0 & 0.0 & 0.0 & 0.0 & 0.0 & 0.0 & 1.3 & 0.0 & 0.0 & 0.0 & 0.0 & 0.2 & 0.0 & 0.1 & 55.9 & 0.0 & 0.0 & 2.9 & 1.0 & 0.0 & 61.4 \\
\hline Other nonmetallic mineral mining & 0.2 & 0.1 & 0.0 & 22.6 & 0.0 & 7.7 & 13.5 & 1.3 & 33.5 & 0.0 & 0.0 & 1.4 & 0.0 & 0.1 & 0.0 & 324.9 & 0.0 & 0.0 & 0.0 & 0.0 & 405.3 \\
\hline $\begin{array}{l}\text { Sand/gravel/clay/refractory mining } \\
\text { Stone mining \& quarrying }\end{array}$ & $\begin{array}{l}0.0 \\
5.8\end{array}$ & $\begin{array}{l}0.7 \\
4.2\end{array}$ & $\begin{array}{l}0.0 \\
0.0\end{array}$ & $\begin{array}{l}0.0 \\
0.0\end{array}$ & $\begin{array}{r}0.7 \\
41.0\end{array}$ & $\begin{array}{l}0.0 \\
0.0\end{array}$ & $\begin{array}{l}2.1 \\
3.9\end{array}$ & $\begin{array}{l}3.6 \\
0.0\end{array}$ & $\begin{array}{r}12.8 \\
0.3\end{array}$ & $\begin{array}{l}0.3 \\
0.0\end{array}$ & $\begin{array}{l}0.2 \\
0.0\end{array}$ & $\begin{array}{l}3.4 \\
0.0\end{array}$ & $\begin{array}{l}0.0 \\
0.0\end{array}$ & $\begin{array}{l}0.0 \\
3.9\end{array}$ & $\begin{array}{l}6.5 \\
0.0\end{array}$ & $\begin{array}{l}34.3 \\
21.6\end{array}$ & $\begin{array}{l}0.0 \\
3.3\end{array}$ & $\begin{array}{l}0.0 \\
0.0\end{array}$ & $\begin{array}{l}1.7 \\
2.1\end{array}$ & $\begin{array}{r}0.0 \\
10.2\end{array}$ & $\begin{array}{l}66.3 \\
96.2\end{array}$ \\
\hline Support activities for oil \& gas operations & 0.3 & 0.0 & 0.0 & 0.0 & 0.0 & 0.0 & 0.2 & 0.0 & 0.1 & 0.0 & 0.0 & 0.0 & 0.0 & 0.0 & 0.0 & 0.0 & 0.0 & 0.1 & 0.0 & 0.0 & 0.8 \\
\hline Support activities for other mining & 0.0 & 0.0 & 0.0 & 0.0 & 0.0 & 0.0 & 0.0 & 0.0 & 0.0 & 0.0 & 0.0 & 0.0 & 0.0 & 0.0 & 0.8 & 0.0 & 0.0 & 0.0 & 0.0 & 0.0 & 0.8 \\
\hline Total & 6.5 & 5.0 & 0.0 & 22.6 & 41.7 & 7.8 & 22.2 & 4.9 & 46.8 & 0.6 & 0.3 & 5.6 & 0.0 & 4.2 & 63.2 & 382.4 & 5.9 & 3.4 & 4.8 & 10.2 & 637.8 \\
\hline
\end{tabular}


Table 11 (continued). Value-added impacts of agriculture and natural resource industries, central region of Florida, 2002 data

Industry Group

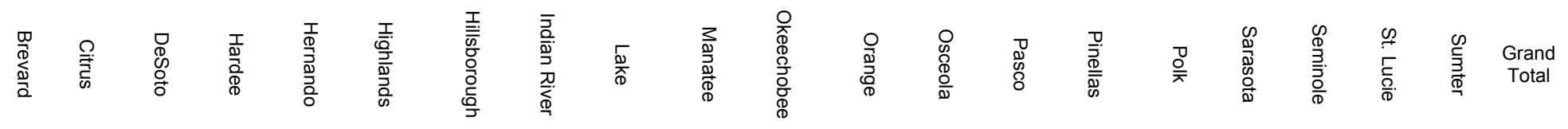

\begin{tabular}{|c|c|c|c|c|c|c|c|c|c|c|c|c|c|c|c|c|c|c|c|c|c|}
\hline \multirow[b]{2}{*}{ Other Crop Farming } & \multirow[b]{2}{*}{0.1} & \multicolumn{20}{|c|}{ (\$Million) } \\
\hline & & 0.2 & 0.3 & 0.9 & 0.7 & 0.8 & 1.6 & 0.0 & 0.8 & 0.8 & 1.5 & 0.2 & 0.4 & 1.4 & 0.0 & 0.9 & 0.1 & 0.1 & 0.0 & 1.6 & 12.4 \\
\hline \multicolumn{22}{|c|}{ Other Food Product Manufacturing } \\
\hline All other food manufacturing & 0.0 & 0.0 & 0.0 & 0.0 & 0.0 & 0.0 & 0.3 & 6.0 & 0.0 & 0.0 & 0.0 & 0.3 & 0.1 & 0.0 & 2.1 & 0.0 & 0.0 & 0.3 & 0.0 & 0.0 & 9.1 \\
\hline Bread/bakery except frozen, mfg & 0.8 & 0.0 & 0.2 & 0.0 & 0.4 & 1.1 & 36.6 & 0.8 & 0.9 & 32.0 & 0.9 & 85.1 & 4.9 & 1.5 & 7.5 & 156.3 & 4.9 & 1.1 & 0.3 & 0.0 & 335.4 \\
\hline Breweries & 0.0 & 0.0 & 0.0 & 0.0 & 0.0 & 0.0 & 52.4 & 0.0 & 0.4 & 0.0 & 0.0 & 0.0 & 0.0 & 0.0 & 0.0 & 48.8 & 0.0 & 0.0 & 0.0 & 0.0 & 101.5 \\
\hline Coffee $\&$ tea manufacturing & 0.0 & 0.0 & 0.0 & 0.0 & 0.0 & 0.0 & 4.9 & 0.0 & 0.0 & 0.0 & 0.0 & 0.2 & 0.0 & 0.0 & 0.4 & 0.0 & 0.0 & 0.0 & 0.0 & 0.0 & 5.5 \\
\hline Cookie \& cracker manufacturing & 0.0 & 0.0 & 0.0 & 0.0 & 0.0 & 0.0 & 112.1 & 0.0 & 0.0 & 0.7 & 0.0 & 0.6 & 0.0 & 0.0 & 0.0 & 1.0 & 0.0 & 0.3 & 0.0 & 0.0 & 114.6 \\
\hline Distilleries & 0.0 & 0.0 & 0.0 & 0.0 & 0.0 & 0.0 & 0.0 & 0.0 & 0.0 & 0.0 & 0.0 & 0.0 & 0.0 & 0.0 & 0.0 & 79.9 & 0.0 & 0.0 & 0.0 & 0.0 & 79.9 \\
\hline Dog/cat food manufacturing & 0.0 & 0.0 & 0.0 & 0.0 & 0.0 & 0.0 & 0.0 & 0.0 & 0.0 & 0.0 & 0.0 & 2.1 & 0.0 & 0.0 & 0.0 & 0.0 & 0.0 & 0.0 & 0.0 & 0.0 & 2.1 \\
\hline Dry pasta manufacturing & 0.0 & 0.0 & 0.0 & 0.0 & 0.0 & 0.0 & 0.1 & 0.0 & 0.0 & 0.0 & 0.0 & 0.0 & 0.0 & 0.0 & 0.0 & 0.0 & 0.0 & 0.0 & 0.0 & 0.0 & 0.1 \\
\hline Flavoring syrup/concentrate mfg & 0.0 & 0.0 & 0.0 & 0.0 & 0.0 & 0.0 & 0.5 & 0.0 & 0.0 & 0.0 & 0.0 & 1.8 & 0.0 & 0.0 & 0.0 & 45.2 & 0.0 & 1.7 & 0.0 & 0.0 & 49.1 \\
\hline Mayonnaise/dressing/sauce mfg & 0.2 & 0.0 & 0.0 & 0.0 & 0.0 & 0.0 & 0.0 & 0.0 & 0.0 & 0.0 & 0.0 & 1.4 & 0.0 & 0.0 & 0.0 & 0.0 & 0.0 & 0.0 & 0.0 & 0.0 & 1.6 \\
\hline Mixes/dough purchased flour & 0.0 & 0.0 & 0.0 & 0.0 & 0.0 & 0.0 & 0.0 & 0.0 & 0.0 & 0.0 & 0.0 & 1.0 & 0.0 & 0.0 & 0.0 & 0.0 & 0.0 & 0.0 & 0.0 & 0.0 & 1.0 \\
\hline Other animal food manufacturing & 0.0 & 0.0 & 0.0 & 0.0 & 0.0 & 0.0 & 3.5 & 0.0 & 2.8 & 0.0 & 15.1 & 0.0 & 11.2 & 0.6 & 0.0 & 40.6 & 0.0 & 0.2 & 0.0 & 1.0 & 74.9 \\
\hline Other snack food manufacturing & 0.0 & 0.0 & 0.0 & 0.0 & 0.0 & 0.0 & 6.8 & 0.0 & 0.0 & 0.0 & 0.0 & 167.2 & 0.0 & 0.0 & 10.4 & 0.0 & 0.0 & 0.0 & 0.0 & 0.0 & 184.4 \\
\hline Roasted nuts/peanut butter mfg & 0.0 & 0.0 & 0.0 & 0.0 & 0.0 & 0.0 & 7.4 & 0.0 & 0.0 & 0.0 & 0.0 & 0.0 & 0.0 & 0.0 & 0.0 & 1.1 & 0.0 & 0.0 & 0.0 & 0.0 & 8.6 \\
\hline Soft drink \& ice manufacturing & 2.1 & 0.0 & 0.0 & 0.0 & 0.0 & 0.0 & 518.2 & 0.0 & 12.0 & 0.0 & 0.0 & 230.0 & 3.0 & 36.3 & 7.2 & 5.0 & 2.6 & 0.0 & 1.6 & 0.0 & 818.1 \\
\hline Spice \& extract manufacturing & 0.2 & 0.0 & 0.0 & 0.0 & 0.0 & 0.0 & 0.0 & 0.0 & 0.0 & 0.0 & 0.0 & 0.6 & 0.0 & 0.5 & 19.8 & 46.8 & 0.0 & 4.3 & 0.0 & 0.0 & 72.4 \\
\hline Wineries & 0.0 & 0.0 & 0.0 & 0.0 & 0.0 & 0.0 & 0.0 & 0.0 & 19.7 & 14.3 & 0.0 & 0.0 & 0.0 & 0.0 & 0.0 & 0.0 & 0.0 & 0.0 & 0.0 & 0.0 & 34.0 \\
\hline Total & 3.5 & 0.0 & 0.2 & 0.0 & 0.4 & 1.1 & 742.8 & 6.8 & 35.8 & 47.0 & 16.0 & 490.1 & 19.1 & 38.9 & 47.5 & 424.6 & 7.5 & 7.9 & 1.9 & 1.0 & $1,892.3$ \\
\hline \multicolumn{22}{|c|}{ Sugarcane Farming, Refined Sugar \& Confections } \\
\hline Confectionery $\mathrm{mfg}$ & 0.6 & 0.0 & 0.0 & 0.0 & 0.0 & 0.0 & 2.0 & 0.0 & 0.0 & 0.0 & 0.0 & 5.3 & 0.0 & 0.0 & 1.5 & 1.0 & 1.9 & 0.0 & 0.7 & 0.0 & 13.0 \\
\hline Non-chocolate confectionery mfg & 0.0 & 0.0 & 0.0 & 0.0 & 0.0 & 0.0 & 59.5 & 0.0 & 0.0 & 6.5 & 0.0 & 0.0 & 0.0 & 0.0 & 15.2 & 13.1 & 0.0 & 8.1 & 0.0 & 0.0 & 102.4 \\
\hline Total & 0.6 & 0.0 & 0.0 & 0.0 & 0.0 & 0.0 & 61.5 & 0.0 & 0.0 & 6.5 & 0.0 & 5.3 & 0.0 & 0.0 & 16.7 & 14.1 & 1.9 & 8.1 & 0.7 & 0.0 & 115.4 \\
\hline Tobacco Farming \& Mfg & 1.3 & 0.0 & 0.0 & 0.0 & 1.1 & 0.0 & 250.9 & 0.0 & 0.0 & 0.0 & 0.0 & 0.2 & 0.0 & 0.0 & 0.1 & 0.0 & 0.6 & 0.4 & 0.0 & 0.0 & 254.5 \\
\hline Wildlife (hunting) & 0.0 & 0.0 & 1.2 & 0.0 & 0.0 & 0.0 & 1.6 & 7.2 & 0.0 & 0.0 & 0.0 & 2.2 & 0.0 & 0.5 & 7.2 & 0.0 & 0.0 & 0.2 & 0.4 & 0.0 & 20.4 \\
\hline Grand Total & 00.8 & 60.2 & 223.8 & 208.6 & 100.2 & 366.6 & $3,279.0$ & 340.4 & 486.0 & $1,424.6$ & 130.6 & $1,629.2$ & 135.8 & 269.6 & 754.8 & $3,098.4$ & 217.2 & 227.5 & 380.3 & 67.5 & $13,601.1$ \\
\hline
\end{tabular}

Source: IMPLAN data for Florida counties (2005) 
Table 12. Labor income impacts of agriculture and natural resource industries, central region of Florida, 2002 data

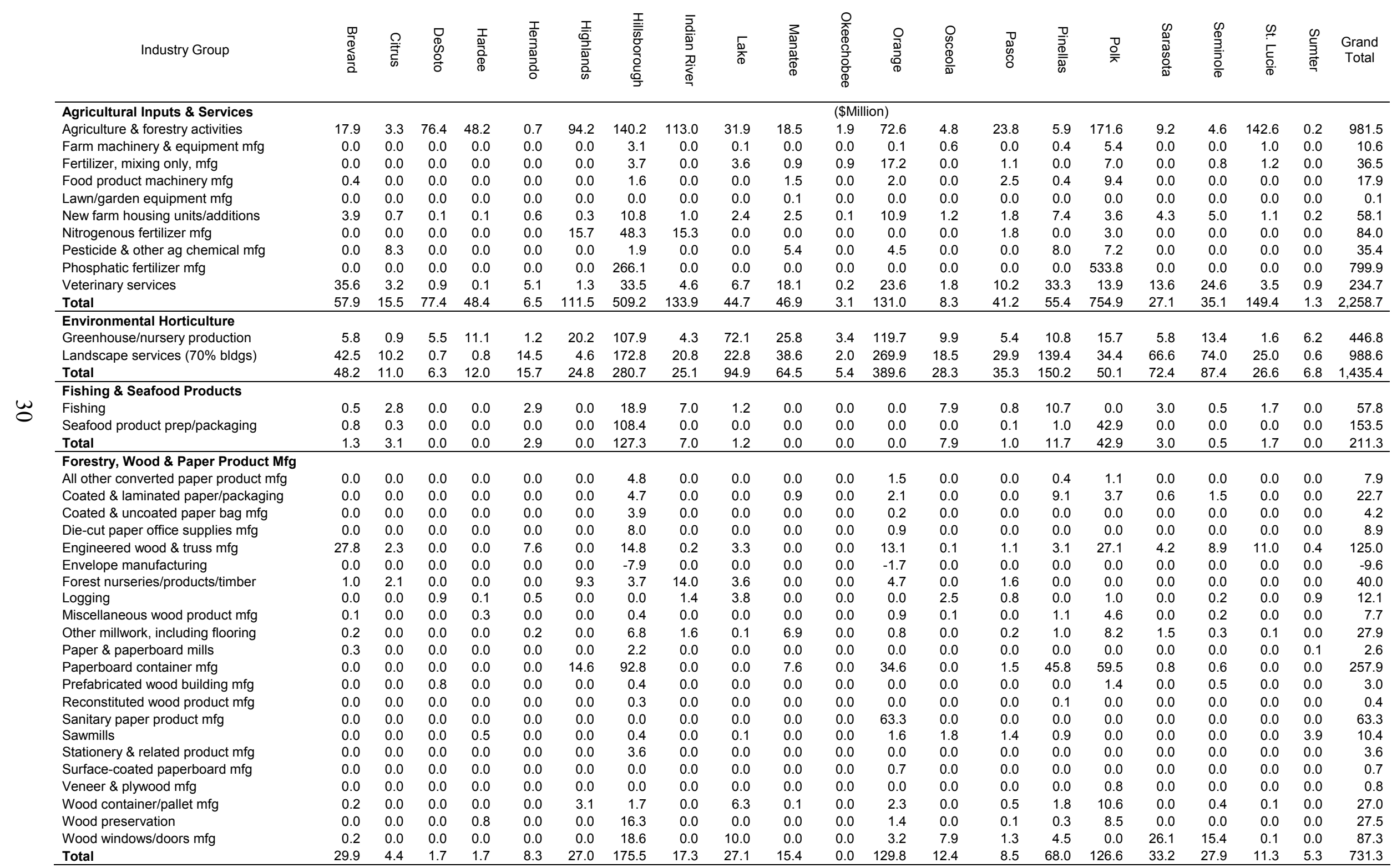


Table 12 (continued). Labor Income Impacts of Agriculture and Natural Resource Industries, Central Region of Florida, 2002 Data

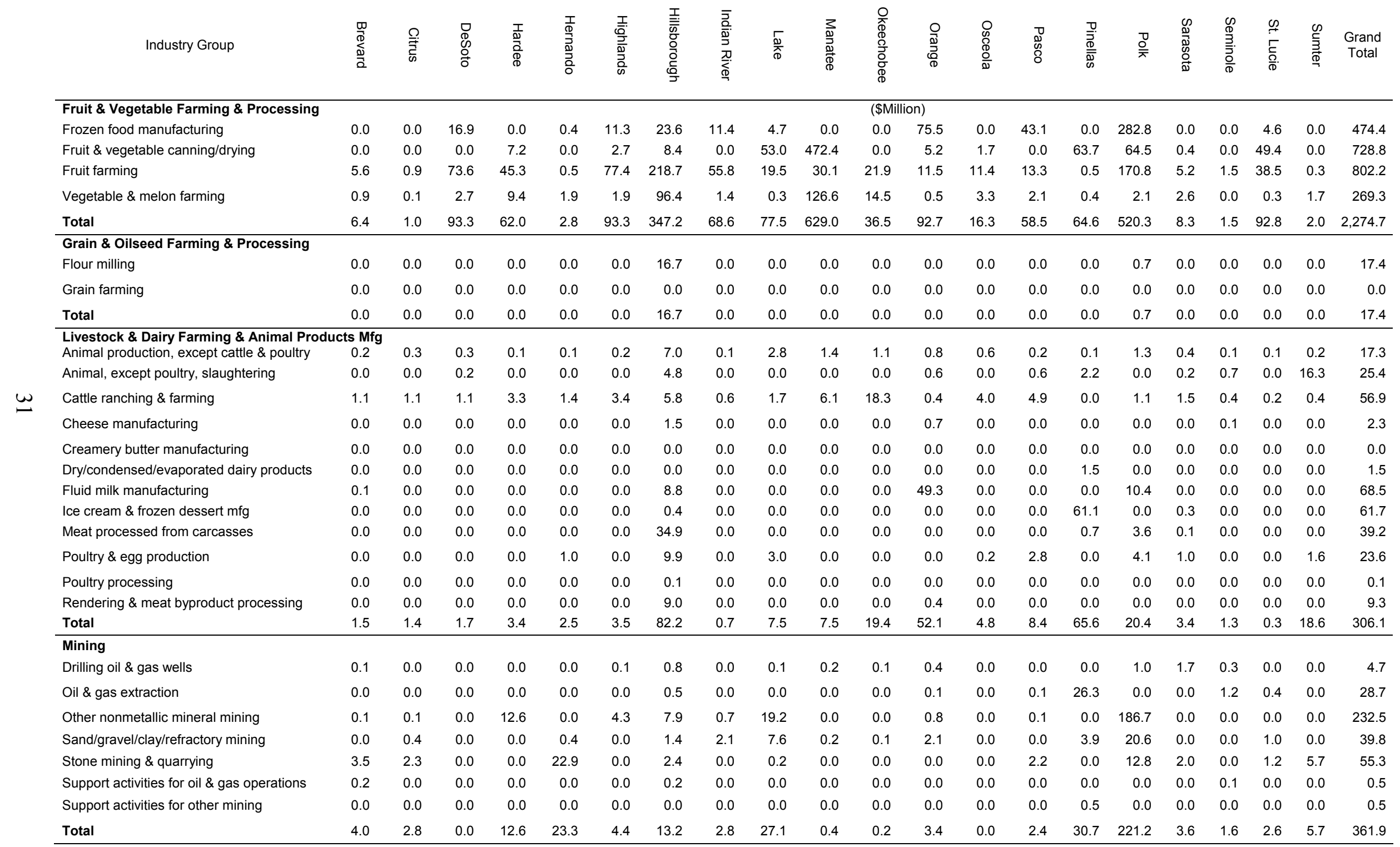


Table 12 (continued). Labor income impacts of agriculture and natural resource industries, central region of Florida, 2002 data

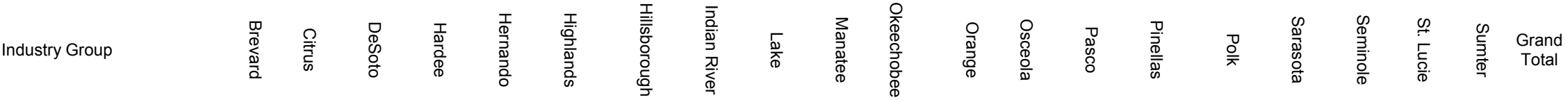

\begin{tabular}{|c|c|c|c|c|c|c|c|c|c|c|c|c|c|c|c|c|c|c|c|c|c|}
\hline \multirow[b]{2}{*}{ Other Crop Farming } & \multicolumn{21}{|c|}{ (\$Million) } \\
\hline & 0.0 & 0.1 & 0.3 & 0.3 & 0.2 & 0.4 & 0.9 & 0.0 & 0.4 & 0.4 & 0.6 & 0.1 & 0.2 & 0.6 & 0.0 & 0.5 & 0.0 & 0.0 & 0.0 & 0.6 & 5.7 \\
\hline \multicolumn{22}{|l|}{ Other Food Product Manufacturing } \\
\hline All other food manufacturing & 0.0 & 0.0 & 0.0 & 0.0 & 0.0 & 0.0 & 0.2 & 3.8 & 0.0 & 0.0 & 0.0 & 0.1 & 0.1 & 0.0 & 1.2 & 0.0 & 0.0 & 0.2 & 0.0 & 0.0 & 5.6 \\
\hline Bread /bakery, except frozen, mfg & 0.5 & 0.0 & 0.0 & 0.0 & 0.2 & 0.6 & 21.0 & 0.5 & 0.5 & 18.6 & 0.5 & 49.9 & 2.8 & 0.9 & 4.3 & 93.6 & 2.8 & 0.6 & 0.2 & 0.0 & 197.5 \\
\hline Breweries & 0.0 & 0.0 & 0.0 & 0.0 & 0.0 & 0.0 & 25.5 & 0.0 & 0.2 & 0.0 & 0.0 & 0.0 & 0.0 & 0.0 & 0.0 & 23.0 & 0.0 & 0.0 & 0.0 & 0.0 & 48.6 \\
\hline Coffee \& tea manufacturing & 0.0 & 0.0 & 0.0 & 0.0 & 0.0 & 0.0 & 2.7 & 0.0 & 0.0 & 0.0 & 0.0 & 0.1 & 0.0 & 0.0 & 0.2 & 0.0 & 0.0 & 0.0 & 0.0 & 0.0 & 3.0 \\
\hline Cookie \& cracker manufacturing & 0.0 & 0.0 & 0.0 & 0.0 & 0.0 & 0.0 & 55.1 & 0.0 & 0.0 & 0.2 & 0.0 & 0.2 & 0.0 & 0.0 & 0.0 & 0.3 & 0.0 & 0.1 & 0.0 & 0.0 & 56.0 \\
\hline Distilleries & 0.0 & 0.0 & 0.0 & 0.0 & 0.0 & 0.0 & 0.0 & 0.0 & 0.0 & 0.0 & 0.0 & 0.0 & 0.0 & 0.0 & 0.0 & 36.8 & 0.0 & 0.0 & 0.0 & 0.0 & 36.8 \\
\hline Dog/cat food manufacturing & 0.0 & 0.0 & 0.0 & 0.0 & 0.0 & 0.0 & 0.0 & 0.0 & 0.0 & 0.0 & 0.0 & 1.2 & 0.0 & 0.0 & 0.0 & 0.0 & 0.0 & 0.0 & 0.0 & 0.0 & 1.2 \\
\hline Dry pasta manufacturing & 0.0 & 0.0 & 0.0 & 0.0 & 0.0 & 0.0 & 0.1 & 0.0 & 0.0 & 0.0 & 0.0 & 0.0 & 0.0 & 0.0 & 0.0 & 0.0 & 0.0 & 0.0 & 0.0 & 0.0 & 0.1 \\
\hline $\begin{array}{l}\text { Flavoring syrup/concentrate } \mathrm{mfg} \\
\text { Mayonnaise/dressing/sauce } \mathrm{mfg} \\
\text { Mixes/dough purchased flour }\end{array}$ & $\begin{array}{l}0.0 \\
0.1 \\
0.0\end{array}$ & $\begin{array}{l}0.0 \\
0.0 \\
0.0\end{array}$ & $\begin{array}{l}0.0 \\
0.0 \\
0.0\end{array}$ & $\begin{array}{l}0.0 \\
0.0 \\
0.0\end{array}$ & $\begin{array}{l}0.0 \\
0.0 \\
0.0\end{array}$ & $\begin{array}{l}0.0 \\
0.0 \\
0.0\end{array}$ & $\begin{array}{l}0.1 \\
0.0 \\
0.0\end{array}$ & $\begin{array}{l}0.0 \\
0.0 \\
0.0\end{array}$ & $\begin{array}{l}0.0 \\
0.0 \\
0.0\end{array}$ & $\begin{array}{l}0.0 \\
0.0 \\
0.0\end{array}$ & $\begin{array}{l}0.0 \\
0.0 \\
0.0\end{array}$ & $\begin{array}{l}0.4 \\
0.4 \\
0.4\end{array}$ & $\begin{array}{l}0.0 \\
0.0 \\
0.0\end{array}$ & $\begin{array}{l}0.0 \\
0.0 \\
0.0\end{array}$ & $\begin{array}{l}0.0 \\
0.0 \\
0.0\end{array}$ & $\begin{array}{r}17.4 \\
0.0 \\
0.0\end{array}$ & $\begin{array}{l}0.0 \\
0.0 \\
0.0\end{array}$ & $\begin{array}{l}0.4 \\
0.0 \\
0.0\end{array}$ & $\begin{array}{l}0.0 \\
0.0 \\
0.0\end{array}$ & $\begin{array}{l}0.0 \\
0.0 \\
0.0\end{array}$ & $\begin{array}{r}18.3 \\
0.5 \\
0.4\end{array}$ \\
\hline Other animal food $\mathrm{mfg}$ & 0.0 & 0.0 & 0.0 & 0.0 & 0.0 & 0.0 & 2.2 & 0.0 & 1.8 & 0.0 & 10.0 & 0.0 & 7.2 & 0.4 & 0.0 & 25.6 & 0.0 & 0.1 & 0.0 & 0.6 & 48.0 \\
\hline Other snack food manufacturing & 0.0 & 0.0 & 0.0 & 0.0 & 0.0 & 0.0 & 2.4 & 0.0 & 0.0 & 0.0 & 0.0 & 84.8 & 0.0 & 0.0 & 3.6 & 0.0 & 0.0 & 0.0 & 0.0 & 0.0 & 90.8 \\
\hline Roasted nuts/peanut butter mfg & 0.0 & 0.0 & 0.0 & 0.0 & 0.0 & 0.0 & 4.2 & 0.0 & 0.0 & 0.0 & 0.0 & 0.0 & 0.0 & 0.0 & 0.0 & 0.6 & 0.0 & 0.0 & 0.0 & 0.0 & 4.8 \\
\hline Soft drink \& ice manufacturing & 1.4 & 0.0 & 0.0 & 0.0 & 0.0 & 0.0 & 325.3 & 0.0 & 7.6 & 0.0 & 0.0 & 141.3 & 1.8 & 22.3 & 4.4 & 2.9 & 1.4 & 0.0 & 1.0 & 0.0 & 509.5 \\
\hline Spice \& extract manufacturing & 0.1 & 0.0 & 0.0 & 0.0 & 0.0 & 0.0 & 0.0 & 0.0 & 0.0 & 0.0 & 0.0 & 0.2 & 0.0 & 0.2 & 8.8 & 22.9 & 0.0 & 1.8 & 0.0 & 0.0 & 34.1 \\
\hline Wineries & 0.0 & 0.0 & 0.0 & 0.0 & 0.0 & 0.0 & 0.0 & 0.0 & 11.7 & 8.3 & 0.0 & 0.0 & 0.0 & 0.0 & 0.0 & 0.0 & 0.0 & 0.0 & 0.0 & 0.0 & 20.0 \\
\hline Total & 2.0 & 0.0 & 0.0 & 0.0 & 0.2 & 0.6 & 438.8 & 4.3 & 21.8 & 27.1 & 10.6 & 279.2 & 11.9 & 23.7 & 22.6 & 223.1 & 4.3 & 3.2 & 1.2 & 0.6 & $1,075.3$ \\
\hline $\begin{array}{l}\text { Sugarcane Farming, Refined Sugar } \\
\text { Confectionery mfg } \\
\text { Non-chocolate confectionery mfg }\end{array}$ & $\begin{array}{c}\text { Confec } \\
0.3 \\
0.0\end{array}$ & $\begin{array}{r}\text { ions } \\
0.0 \\
0.0\end{array}$ & $\begin{array}{l}0.0 \\
0.0\end{array}$ & $\begin{array}{l}0.0 \\
0.0\end{array}$ & $\begin{array}{l}0.0 \\
0.0\end{array}$ & $\begin{array}{l}0.0 \\
0.0\end{array}$ & $\begin{array}{r}1.3 \\
32.7\end{array}$ & $\begin{array}{l}0.0 \\
0.0\end{array}$ & $\begin{array}{l}0.0 \\
0.0\end{array}$ & $\begin{array}{l}0.0 \\
3.6\end{array}$ & $\begin{array}{l}0.0 \\
0.0\end{array}$ & $\begin{array}{l}2.9 \\
0.0\end{array}$ & $\begin{array}{l}0.0 \\
0.0\end{array}$ & $\begin{array}{l}0.0 \\
0.0\end{array}$ & $\begin{array}{l}0.8 \\
8.3\end{array}$ & $\begin{array}{l}0.6 \\
7.0\end{array}$ & $\begin{array}{l}1.0 \\
0.0\end{array}$ & $\begin{array}{l}0.0 \\
4.4\end{array}$ & $\begin{array}{l}0.4 \\
0.0\end{array}$ & $\begin{array}{l}0.0 \\
0.0\end{array}$ & $\begin{array}{r}7.3 \\
56.0\end{array}$ \\
\hline Total & 0.3 & 0.0 & 0.0 & 0.0 & 0.0 & 0.0 & 34.0 & 0.0 & 0.0 & 3.6 & 0.0 & 2.9 & 0.0 & 0.0 & 9.1 & 7.6 & 1.0 & 4.4 & 0.4 & 0.0 & 63.3 \\
\hline Tobacco Farming \& Manufacturing & 0.4 & 0.0 & 0.0 & 0.0 & 0.3 & 0.0 & 106.1 & 0.0 & 0.0 & 0.0 & 0.0 & 0.1 & 0.0 & 0.0 & 0.0 & 0.0 & 0.1 & 0.1 & 0.0 & 0.0 & 107.0 \\
\hline Wildlife (hunting) & 0.0 & 0.0 & 1.4 & 0.0 & 0.0 & 0.0 & 0.9 & 4.7 & 0.0 & 0.0 & 0.0 & 1.1 & 0.0 & 0.2 & 3.5 & 0.0 & 0.0 & 0.1 & 0.1 & 0.0 & 12.0 \\
\hline Grand Total & 151.9 & 39.3 & 181.9 & 140.3 & 62.8 & 265.5 & $2,132.7$ & 264.4 & 302.2 & 794.9 & 75.8 & $1,082.1$ & 90.1 & 179.7 & 481.4 & $1,968.2$ & 156.5 & 163.2 & 286.4 & 41.0 & $8,860.2$ \\
\hline
\end{tabular}

Source: IMPLAN data for Florida counties (2005) 
Table 13. Indirect business tax impacts of agriculture and natural resource industries, central region of Florida, 2002 data

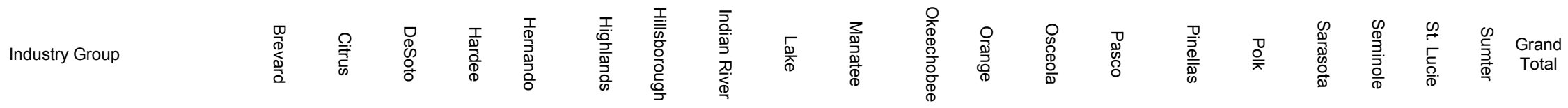

\begin{tabular}{|c|c|c|c|c|c|c|c|c|c|c|c|c|c|c|c|c|c|c|c|c|c|}
\hline \multicolumn{2}{|l|}{ Agricultural Inputs \& Services } & \multicolumn{20}{|c|}{ (\$Million) } \\
\hline Agriculture \& forestry activities & 0.8 & 0.1 & 2.7 & 1.8 & 0.0 & 4.3 & 6.1 & 4.9 & 1.3 & 0.2 & 0.0 & 3.5 & 0.2 & 1.0 & 0.4 & 10.3 & 0.5 & 1.6 & 8.0 & 0.0 & 48.1 \\
\hline Farm machinery \& equipment mfg & 0.0 & 0.0 & 0.0 & 0.0 & 0.0 & 0.0 & 0.2 & 0.0 & 0.0 & 0.0 & 0.0 & 0.0 & 0.0 & 0.0 & 0.0 & 0.3 & 0.0 & 0.0 & 0.0 & 0.0 & 0.6 \\
\hline Fertilizer, mixing only, mfg & 0.0 & 0.0 & 0.0 & 0.0 & 0.0 & 0.0 & 0.2 & 0.0 & 0.2 & 0.1 & 0.1 & 1.2 & 0.0 & 0.1 & 0.0 & 0.5 & 0.0 & 0.0 & 0.1 & 0.0 & 2.5 \\
\hline Food product machinery $\mathrm{mfg}$ & 0.0 & 0.0 & 0.0 & 0.0 & 0.0 & 0.0 & 0.1 & 0.0 & 0.0 & 0.1 & 0.0 & 0.2 & 0.0 & 0.1 & 0.0 & 0.4 & 0.0 & 0.0 & 0.0 & 0.0 & 0.9 \\
\hline Lawn/garden equipment mfg & 0.0 & 0.0 & 0.0 & 0.0 & 0.0 & 0.0 & 0.0 & 0.0 & 0.0 & 0.0 & 0.0 & 0.0 & 0.0 & 0.0 & 0.0 & 0.0 & 0.0 & 0.0 & 0.0 & 0.0 & 0.0 \\
\hline New farm housing units/additions & 0.1 & 0.0 & 0.0 & 0.0 & 0.0 & 0.0 & 0.2 & 0.0 & 0.1 & 0.1 & 0.0 & 0.2 & 0.0 & 0.0 & 0.2 & 0.1 & 0.1 & 0.1 & 0.0 & 0.0 & 1.2 \\
\hline Nitrogenous fertilizer $\mathrm{mfg}$ & 0.0 & 0.0 & 0.0 & 0.0 & 0.0 & 1.2 & 4.1 & 1.2 & 0.0 & 0.0 & 0.0 & 0.0 & 0.0 & 0.1 & 0.0 & 0.3 & 0.0 & 0.0 & 0.0 & 0.0 & 7.0 \\
\hline Pesticide and other ag chemical mg & 0.0 & 0.7 & 0.0 & 0.0 & 0.0 & 0.0 & 0.2 & 0.0 & 0.0 & 0.5 & 0.0 & 0.4 & 0.0 & 0.0 & 0.8 & 0.6 & 0.0 & 0.0 & 0.0 & 0.0 & 3.2 \\
\hline Phosphatic fertilizer mfg & 0.0 & 0.0 & 0.0 & 0.0 & 0.0 & 0.0 & 26.1 & 0.0 & 0.0 & 0.0 & 0.0 & 0.0 & 0.0 & 0.0 & 0.0 & 53.8 & 0.0 & 0.0 & 0.0 & 0.0 & 79.9 \\
\hline Veterinary services & 3.2 & 0.3 & 0.1 & 0.0 & 0.5 & 0.1 & 2.6 & 0.4 & 0.6 & 2.0 & 0.0 & 1.7 & 0.1 & 0.8 & 2.8 & 1.2 & 1.1 & 8.8 & 0.3 & 0.1 & 26.7 \\
\hline Total & 4.1 & 1.1 & 2.8 & 1.8 & 0.5 & 5.6 & 39.9 & 6.5 & 2.2 & 3.0 & 0.1 & 7.3 & 0.4 & 2.1 & 4.2 & 67.6 & 1.7 & 10.6 & 8.5 & 0.1 & 170.1 \\
\hline \multicolumn{22}{|l|}{ Environmental Horticulture } \\
\hline Greenhouse/nursery production & 0.3 & 0.0 & 0.3 & 0.7 & 0.0 & 1.4 & 7.4 & 0.2 & 5.2 & 1.8 & 0.2 & 7.6 & 0.7 & 0.2 & 0.7 & 1.0 & 0.4 & 4.2 & 0.1 & 0.4 & 32.7 \\
\hline Landscape services ( $70 \%$ bldgs) & 1.9 & 0.6 & 0.0 & 0.0 & 0.9 & 0.2 & 9.7 & 1.3 & 1.4 & 2.7 & 0.1 & 15.7 & 1.0 & 1.7 & 9.7 & 1.7 & 4.1 & 19.0 & 1.7 & 0.0 & 73.3 \\
\hline Total & 2.1 & 0.6 & 0.3 & 0.7 & 0.9 & 1.6 & 17.1 & 1.5 & 6.5 & 4.4 & 0.3 & 23.3 & 1.6 & 1.9 & 10.4 & 2.7 & 4.5 & 23.1 & 1.7 & 0.4 & 105.9 \\
\hline \multicolumn{22}{|l|}{ Fishing \& Seafood Products } \\
\hline Fishing & 0.0 & 0.2 & 0.0 & 0.0 & 0.2 & 0.0 & 1.4 & 0.5 & 0.1 & 0.0 & 0.0 & 0.0 & 0.6 & 0.1 & 0.8 & 0.0 & 0.2 & 0.2 & 0.1 & 0.0 & 4.4 \\
\hline Seafood product prep/packaging & 0.0 & 0.0 & 0.0 & 0.0 & 0.0 & 0.0 & 11.8 & 0.0 & 0.0 & 0.0 & 0.0 & 0.0 & 0.0 & 0.0 & 0.0 & 4.4 & 0.0 & 0.0 & 0.0 & 0.0 & 16.3 \\
\hline Total & 0.1 & 0.2 & 0.0 & 0.0 & 0.2 & 0.0 & 13.2 & 0.5 & 0.1 & 0.0 & 0.0 & 0.0 & 0.6 & 0.1 & 0.9 & 4.4 & 0.2 & 0.2 & 0.1 & 0.0 & 20.7 \\
\hline \multicolumn{22}{|l|}{ Forestry, Wood \& Paper Product Mfg } \\
\hline All other converted paper product mfg & 0.0 & 0.0 & 0.0 & 0.0 & 0.0 & 0.0 & 0.5 & 0.0 & 0.0 & 0.0 & 0.0 & 0.2 & 0.0 & 0.0 & 0.0 & 0.1 & 0.0 & 0.0 & 0.0 & 0.0 & 0.8 \\
\hline Coated \& laminated paper/packaging & 0.0 & 0.0 & 0.0 & 0.0 & 0.0 & 0.0 & 0.5 & 0.0 & 0.0 & 0.1 & 0.0 & 0.3 & 0.0 & 0.0 & 1.0 & 0.4 & 0.1 & 1.0 & 0.0 & 0.0 & 3.5 \\
\hline Coated \& uncoated paper bag $\mathrm{mfg}$ & 0.0 & 0.0 & 0.0 & 0.0 & 0.0 & 0.0 & 0.4 & 0.0 & 0.0 & 0.0 & 0.0 & 0.0 & 0.0 & 0.0 & 0.0 & 0.0 & 0.0 & 0.0 & 0.0 & 0.0 & 0.4 \\
\hline Die-cut paper office supplies mfg & 0.0 & 0.0 & 0.0 & 0.0 & 0.0 & 0.0 & 0.8 & 0.0 & 0.0 & 0.0 & 0.0 & 0.1 & 0.0 & 0.0 & 0.0 & 0.0 & 0.0 & 0.0 & 0.0 & 0.0 & 0.9 \\
\hline Engineered wood \& truss $\mathrm{mfg}$ & 1.8 & 0.1 & 0.0 & 0.0 & 0.5 & 0.0 & 0.8 & 0.0 & 0.1 & 0.0 & 0.0 & 0.6 & 0.0 & 0.0 & 0.1 & 2.0 & 0.2 & 2.7 & 0.9 & 0.0 & 9.7 \\
\hline Envelope manufacturing & 0.0 & 0.0 & 0.0 & 0.0 & 0.0 & 0.0 & -0.1 & 0.0 & 0.0 & 0.0 & 0.0 & 0.0 & 0.0 & 0.0 & 0.0 & 0.0 & 0.0 & 0.0 & 0.0 & 0.0 & -0.1 \\
\hline Forest nurseries/products/timber & 0.1 & 0.3 & 0.0 & 0.0 & 0.0 & 0.8 & 0.3 & 1.1 & 0.3 & 0.0 & 0.0 & 0.5 & 0.0 & 0.1 & 0.0 & 0.0 & 0.0 & 0.0 & 0.0 & 0.0 & 3.6 \\
\hline Logging & 0.0 & 0.0 & 0.1 & 0.0 & 0.0 & 0.0 & 0.0 & 0.1 & 0.3 & 0.0 & 0.0 & 0.0 & 0.2 & 0.0 & 0.0 & 0.1 & 0.0 & 0.1 & 0.0 & 0.1 & 0.9 \\
\hline Miscellaneous wood product mfg & 0.0 & 0.0 & 0.0 & 0.0 & 0.0 & 0.0 & 0.0 & 0.0 & 0.0 & 0.0 & 0.0 & 0.0 & 0.0 & 0.0 & 0.0 & 0.4 & 0.0 & 0.0 & 0.0 & 0.0 & 0.5 \\
\hline Other millwork, including flooring & 0.0 & 0.0 & 0.0 & 0.0 & 0.0 & 0.0 & 0.1 & 0.1 & 0.0 & 0.3 & 0.0 & 0.0 & 0.0 & 0.0 & 0.0 & 0.4 & 0.0 & 0.0 & 0.0 & 0.0 & 1.0 \\
\hline Paper \& paperboard mills & 0.0 & 0.0 & 0.0 & 0.0 & 0.0 & 0.0 & 0.3 & 0.0 & 0.0 & 0.0 & 0.0 & 0.0 & 0.0 & 0.0 & 0.0 & 0.0 & 0.0 & 0.0 & 0.0 & 0.0 & 0.3 \\
\hline Paperboard container $\mathrm{mfg}$ & 0.0 & 0.0 & 0.0 & 0.0 & 0.0 & 1.2 & 8.1 & 0.0 & 0.0 & 0.7 & 0.0 & 2.9 & 0.0 & 0.1 & 4.5 & 5.2 & 0.1 & 0.3 & 0.0 & 0.0 & 23.0 \\
\hline Prefabricated wood building $\mathrm{mfg}$ & 0.0 & 0.0 & 0.0 & 0.0 & 0.0 & 0.0 & 0.0 & 0.0 & 0.0 & 0.0 & 0.0 & 0.0 & 0.0 & 0.0 & 0.0 & 0.1 & 0.0 & 0.3 & 0.0 & 0.0 & 0.5 \\
\hline Reconstituted wood product mfg & 0.0 & 0.0 & 0.0 & 0.0 & 0.0 & 0.0 & 0.0 & 0.0 & 0.0 & 0.0 & 0.0 & 0.0 & 0.0 & 0.0 & 0.0 & 0.0 & 0.0 & 0.0 & 0.0 & 0.0 & 0.0 \\
\hline Sanitary paper product mfg & 0.0 & 0.0 & 0.0 & 0.0 & 0.0 & 0.0 & 0.0 & 0.0 & 0.0 & 0.0 & 0.0 & 5.3 & 0.0 & 0.0 & 0.0 & 0.0 & 0.0 & 0.0 & 0.0 & 0.0 & 5.3 \\
\hline Sawmills & 0.0 & 0.0 & 0.0 & 0.0 & 0.0 & 0.0 & 0.0 & 0.0 & 0.0 & 0.0 & 0.0 & 0.1 & 0.1 & 0.1 & 0.0 & 0.0 & 0.0 & 0.0 & 0.0 & 0.3 & 0.6 \\
\hline Stationery \& related product $\mathrm{mfg}$ & 0.0 & 0.0 & 0.0 & 0.0 & 0.0 & 0.0 & 0.4 & 0.0 & 0.0 & 0.0 & 0.0 & 0.0 & 0.0 & 0.0 & 0.0 & 0.0 & 0.0 & 0.0 & 0.0 & 0.0 & 0.4 \\
\hline Surface-coated paperboard $\mathrm{mfg}$ & 0.0 & 0.0 & 0.0 & 0.0 & 0.0 & 0.0 & 0.0 & 0.0 & 0.0 & 0.0 & 0.0 & 0.1 & 0.0 & 0.0 & 0.0 & 0.0 & 0.0 & 0.0 & 0.0 & 0.0 & 0.1 \\
\hline Veneer \& plywood mfg & 0.0 & 0.0 & 0.0 & 0.0 & 0.0 & 0.0 & 0.0 & 0.0 & 0.0 & 0.0 & 0.0 & 0.0 & 0.0 & 0.0 & 0.0 & 0.0 & 0.0 & 0.0 & 0.0 & 0.0 & 0.0 \\
\hline Wood container/pallet $\mathrm{mfg}$ & 0.0 & 0.0 & 0.0 & 0.0 & 0.0 & 0.2 & 0.0 & 0.0 & 0.4 & 0.0 & 0.0 & 0.0 & 0.0 & 0.0 & 0.0 & 0.6 & 0.0 & 0.0 & 0.0 & 0.0 & 1.2 \\
\hline Wood preservation & 0.0 & 0.0 & 0.0 & 0.1 & 0.0 & 0.0 & 1.8 & 0.0 & 0.0 & 0.0 & 0.0 & 0.1 & 0.0 & 0.0 & 0.0 & 0.9 & 0.0 & 0.0 & 0.0 & 0.0 & 2.9 \\
\hline Wood windows/doors $\mathrm{mfg}$ & 0.0 & 0.0 & 0.0 & 0.0 & 0.0 & 0.0 & 0.9 & 0.0 & 0.6 & 0.0 & 0.0 & 0.1 & 0.6 & 0.0 & 0.1 & 0.0 & 1.9 & 5.2 & 0.0 & 0.0 & 9.4 \\
\hline Total & 2.0 & 0.4 & 0.1 & 0.1 & 0.5 & 2.1 & 14.9 & 1.3 & 1.8 & 1.1 & 0.0 & 10.1 & 0.9 & 0.4 & 5.9 & 10.1 & 2.2 & 9.7 & 0.9 & 0.4 & 64.9 \\
\hline
\end{tabular}


Table 13 (continued). Indirect business tax impacts of agriculture and natural resource industries, central region of Florida, 2002 data

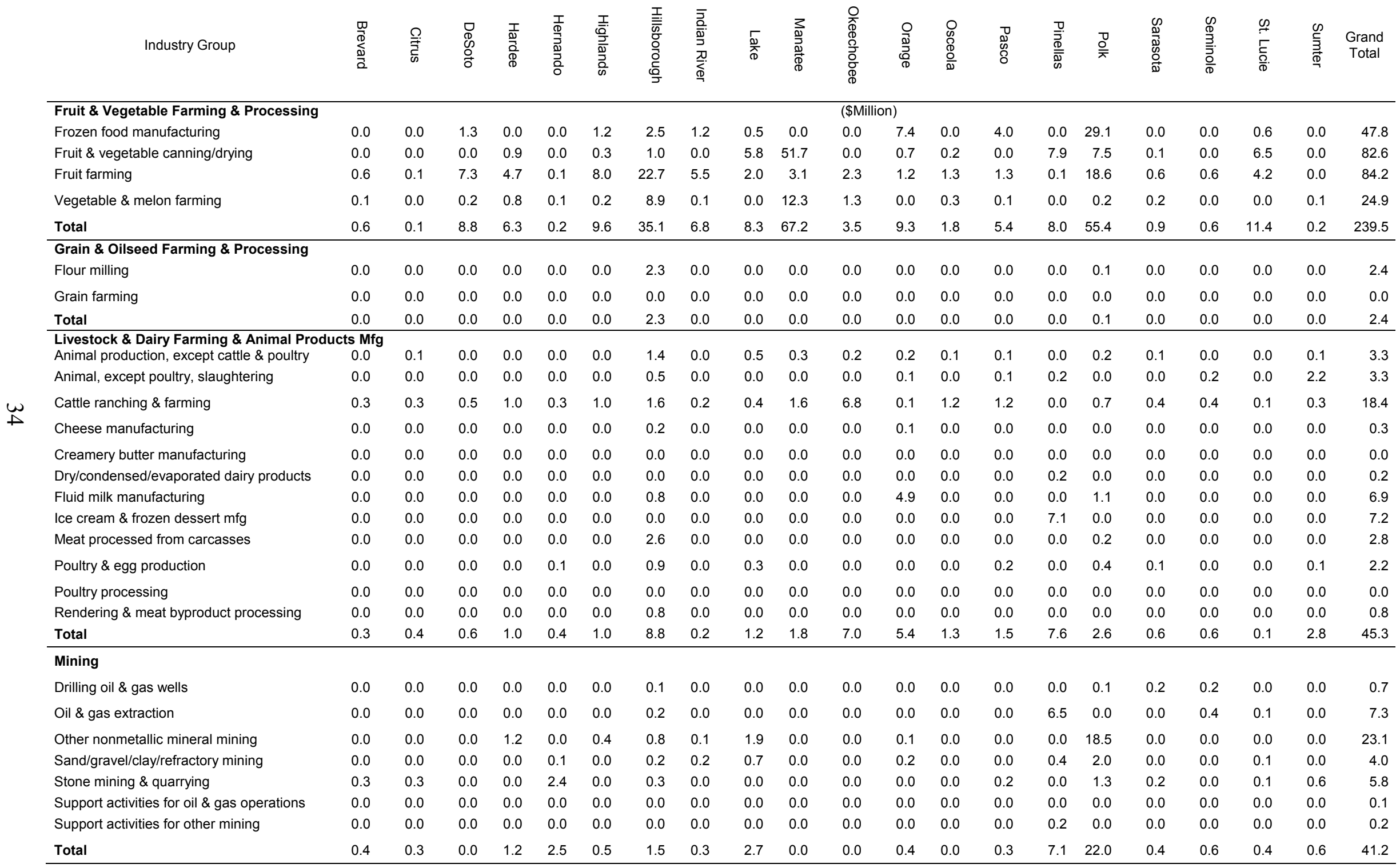


Table 13 (continued). Indirect business tax impacts of agriculture and natural resource industries, central region of Florida, 2002 data

Industry Group

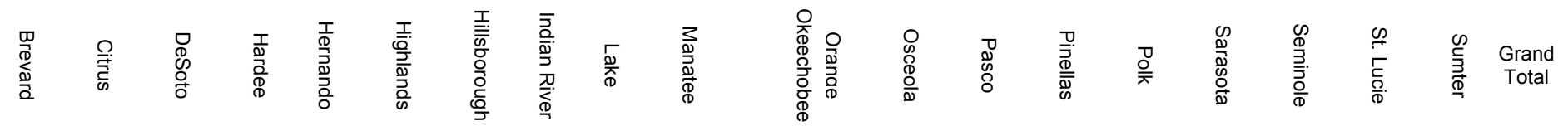

\begin{tabular}{|c|c|c|c|c|c|c|c|c|c|c|c|c|c|c|c|c|c|c|c|c|c|}
\hline \multirow[b]{2}{*}{ Other Crop Farming } & \multicolumn{21}{|c|}{ (\$Million) } \\
\hline & 0.0 & 0.0 & 0.0 & 0.1 & 0.0 & 0.0 & 0.1 & 0.0 & 0.0 & 0.1 & 0.1 & 0.0 & 0.0 & 0.1 & 0.0 & 0.1 & 0.0 & 0.0 & 0.0 & 0.1 & 0.8 \\
\hline \multicolumn{22}{|l|}{ Other Food Product Manufacturing } \\
\hline All other food manufacturing & 0.0 & 0.0 & 0.0 & 0.0 & 0.0 & 0.0 & 0.0 & 0.3 & 0.0 & 0.0 & 0.0 & 0.0 & 0.0 & 0.0 & 0.1 & 0.0 & 0.0 & 0.1 & 0.0 & 0.0 & 0.5 \\
\hline Bread/bakery, except frozen, $\mathrm{mfg}$ & 0.0 & 0.0 & 0.0 & 0.0 & 0.0 & 0.0 & 0.6 & 0.0 & 0.0 & 1.0 & 0.0 & 2.3 & 0.1 & 0.0 & 0.1 & 6.6 & 0.1 & 0.0 & 0.0 & 0.0 & 11.0 \\
\hline Breweries & 0.0 & 0.0 & 0.0 & 0.0 & 0.0 & 0.0 & 10.1 & 0.0 & 0.1 & 0.0 & 0.0 & 0.0 & 0.0 & 0.0 & 0.0 & 9.5 & 0.0 & 0.0 & 0.0 & 0.0 & 19.7 \\
\hline Coffee \& tea manufacturing & 0.0 & 0.0 & 0.0 & 0.0 & 0.0 & 0.0 & 0.4 & 0.0 & 0.0 & 0.0 & 0.0 & 0.0 & 0.0 & 0.0 & 0.0 & 0.0 & 0.0 & 0.0 & 0.0 & 0.0 & 0.5 \\
\hline Cookie \& cracker manufacturing & 0.0 & 0.0 & 0.0 & 0.0 & 0.0 & 0.0 & 5.5 & 0.0 & 0.0 & 0.0 & 0.0 & 0.0 & 0.0 & 0.0 & 0.0 & 0.0 & 0.0 & 0.0 & 0.0 & 0.0 & 5.6 \\
\hline Distilleries & 0.0 & 0.0 & 0.0 & 0.0 & 0.0 & 0.0 & 0.0 & 0.0 & 0.0 & 0.0 & 0.0 & 0.0 & 0.0 & 0.0 & 0.0 & 30.5 & 0.0 & 0.0 & 0.0 & 0.0 & 30.5 \\
\hline Dog/cat food manufacturing & 0.0 & 0.0 & 0.0 & 0.0 & 0.0 & 0.0 & 0.0 & 0.0 & 0.0 & 0.0 & 0.0 & 0.1 & 0.0 & 0.0 & 0.0 & 0.0 & 0.0 & 0.0 & 0.0 & 0.0 & 0.1 \\
\hline Flavoring syrup/concentrate mfg & 0.0 & 0.0 & 0.0 & 0.0 & 0.0 & 0.0 & 0.0 & 0.0 & 0.0 & 0.0 & 0.0 & 0.0 & 0.0 & 0.0 & 0.0 & 1.6 & 0.0 & 0.1 & 0.0 & 0.0 & 1.8 \\
\hline Other animal food manufacturing & 0.0 & 0.0 & 0.0 & 0.0 & 0.0 & 0.0 & 0.3 & 0.0 & 0.2 & 0.0 & 1.3 & 0.0 & 1.0 & 0.0 & 0.0 & 3.5 & 0.0 & 0.1 & 0.0 & 0.1 & 6.5 \\
\hline Other snack food manufacturing & 0.0 & 0.0 & 0.0 & 0.0 & 0.0 & 0.0 & 0.2 & 0.0 & 0.0 & 0.0 & 0.0 & 9.5 & 0.0 & 0.0 & 0.3 & 0.0 & 0.0 & 0.0 & 0.0 & 0.0 & 9.9 \\
\hline Roasted nuts/peanut butter mfg & 0.0 & 0.0 & 0.0 & 0.0 & 0.0 & 0.0 & 0.6 & 0.0 & 0.0 & 0.0 & 0.0 & 0.0 & 0.0 & 0.0 & 0.0 & 0.1 & 0.0 & 0.0 & 0.0 & 0.0 & 0.6 \\
\hline Soft drink \& ice manufacturing & 0.1 & 0.0 & 0.0 & 0.0 & 0.0 & 0.0 & 31.0 & 0.0 & 0.7 & 0.0 & 0.0 & 13.9 & 0.2 & 2.0 & 0.5 & 0.3 & 0.2 & 0.0 & 0.1 & 0.0 & 49.1 \\
\hline Spice \& extract manufacturing & 0.0 & 0.0 & 0.0 & 0.0 & 0.0 & 0.0 & 0.0 & 0.0 & 0.0 & 0.0 & 0.0 & 0.0 & 0.0 & 0.0 & 0.8 & 2.1 & 0.0 & 0.7 & 0.0 & 0.0 & 3.6 \\
\hline Wineries & 0.0 & 0.0 & 0.0 & 0.0 & 0.0 & 0.0 & 0.0 & 0.0 & 3.5 & 2.8 & 0.0 & 0.0 & 0.0 & 0.0 & 0.0 & 0.0 & 0.0 & 0.0 & 0.0 & 0.0 & 6.3 \\
\hline Total & 0.2 & 0.0 & 0.0 & 0.0 & 0.0 & 0.0 & 48.7 & 0.3 & 4.6 & 3.8 & 1.3 & 26.0 & 1.3 & 2.1 & 1.8 & 54.3 & 0.3 & 0.9 & 0.1 & 0.1 & 145.7 \\
\hline \multicolumn{22}{|c|}{ Sugarcane Farming, Refined Sugar \& Confections } \\
\hline Confectionery $\mathrm{mfg}$ & 0.0 & 0.0 & 0.0 & 0.0 & 0.0 & 0.0 & 0.2 & 0.0 & 0.0 & 0.0 & 0.0 & 0.3 & 0.0 & 0.0 & 0.1 & 0.1 & 0.1 & 0.0 & 0.1 & 0.0 & 0.8 \\
\hline Non-chocolate confectionery $\mathrm{mfg}$ & 0.0 & 0.0 & 0.0 & 0.0 & 0.0 & 0.0 & 3.5 & 0.0 & 0.0 & 0.5 & 0.0 & 0.0 & 0.0 & 0.0 & 1.0 & 0.8 & 0.0 & 3.3 & 0.0 & 0.0 & 9.1 \\
\hline Total & 0.0 & 0.0 & 0.0 & 0.0 & 0.0 & 0.0 & 3.7 & 0.0 & 0.0 & 0.5 & 0.0 & 0.3 & 0.0 & 0.0 & 1.1 & 0.8 & 0.1 & 3.3 & 0.1 & 0.0 & 9.8 \\
\hline Tobacco Farming \& Manufacturing & 0.0 & 0.0 & 0.0 & 0.0 & 0.0 & 0.0 & 8.7 & 0.0 & 0.0 & 0.0 & 0.0 & 0.0 & 0.0 & 0.0 & 0.0 & 0.0 & 0.0 & 0.0 & 0.0 & 0.0 & 8.7 \\
\hline Wildlife (hunting) & 0.0 & 0.0 & 0.2 & 0.0 & 0.0 & 0.0 & 0.2 & 0.7 & 0.0 & 0.0 & 0.0 & 0.2 & 0.0 & 0.1 & 0.8 & 0.0 & 0.0 & 0.0 & 0.1 & 0.0 & 2.3 \\
\hline Grand Total & 9.9 & 3.1 & 12.8 & 11.3 & 5.3 & 20.5 & 194.0 & 18.1 & 27.3 & 81.8 & 12.4 & 82.2 & 7.9 & 14.0 & 47.7 & 220.1 & 10.9 & 49.9 & 23.3 & 4.6 & 857.2 \\
\hline
\end{tabular}

Source: IMPLAN data for Florida counties (2005) 